\title{
Investigação sobre a interação entre os campos escalar e gravitacional no espaço não comutativo.
}

\author{
Manoel Reinaldo Elias Filho
}

ORIENTADOR: Prof. Dr. Fernado Tadeu Caldeira Brandt

Dissertação de Mestrado submetida ao Instituto de Física da Universidade de São Paulo como parte dos requisitos para a obtenção do grau de Mestre em Ciências.

São Paulo 2006 


\section{Resumo}

A formulação de uma teoria da relatividade geral em espaços não comutativos tem sido investigada usando diferentes abordagens atualmente. Neste contexto, é feita uma revisão do formalismo que descreve a não comutatividade do espaço-tempo. A seguir, são investigadas as interações entre os campos do gráviton e o bóson escalar, formuladas em um espaço comutativo mostrando-se um método alternativo para reescrever a ação desta interação. Finalmente, utilizando-se este método, obtém-se o termo dominante da ação de interação entre os campos do gráviton e escalar em espaços não comutativos. Deste modo, somos levados a considerar transformações restritas com parâmetro infinitesimal $\tilde{w}^{\mu}(x)$ tal que $\partial_{\mu} \tilde{w}^{\mu}=0$. Temos assim a versão não comutativa da gravidade unimodular. 


\begin{abstract}
The formulation of a general relativity theory on noncommutative spaces has been investigated using many different approaches. In this context, a revision of the formalism that describes noncommutative spacetime is made. Next, the interactions between the fields of graviton and scalar is investigated in a commutative space showing an alternative method to rewrite the action of this interaction. Finally, using this method, one obtains the dominant term of the action of graviton interacting with a scalar particle in a noncomutative space. In this way, we are led to consider restricted transformations with infinitesimal parameter $\tilde{w}^{\mu}(x)$, such that, $\partial_{\mu} \tilde{w}^{\mu}=0$. Thus we have the noncommutative version of unimodular gravity.
\end{abstract}




\section{Agradecimentos}

Ao Professor Dr. Fernando Tadeu Caldeira Brandt, pela orientação, paciência e compreensão.

À CAPES, pelo apoio financeiro.

À amiga Tiana pelos momentos de alegria e pelo acolhimento em São Paulo, fazendo com que as preocupações de moradia ficassem em terceiro plano.

Aos paraenses do IFUSP que neste período foram mais que amigos. Cada um deles possuem vários momentos especiais gravados em minha memória, e seria necessário um livro para escrevê-los. São eles: Alexandre Guimarães (Fidel), Edson Akira, Andrey Martins, Cléber de Brito, Karlúcio Castelo Branco, Leônidas Melo, Damião, Charles e em particular ao meu amigo irmão Reginaldo de Jesus Costa Farias que abraçou a ídeia de olhar a curvatura da esfera, partindo de uma realidade de quarto mundo descendo do norte pra cidade grande.

Aos amigos não paraenses do IFUSP que foram importantes para minha adaptação em São Paulo, pelas discussões relacionadas as listas de exercícios, à física e pelo aprendizado das inumeras culturas deste Brasil. São eles: Leonardo (Digníssimo!!!), André Lehum, Kleto, Sabrina, Fábio, Marcelo Pires, Tiago Adorno, ..., e em particular ao meu companheiro de sala Cleber Muramoto, pela amizade, pelas discussões relacionadas a física e papos sobre futebol, lugares, mulheres, etc...

Aos meus professores na UFPA, Licurgo Brito, Van Sérgio, Sérgio Vizeu, Marcelo Lima, Wanda Ignácio, Bassalo e Paulo de Tarso, que sempre me incentivaram durante minha graduação.

Em especial quero agradecer, a uma pessoa que se encontra apenas nos pensamentos dos que o conheceram e nas lembranças que tenho dos momentos que compartilhamos na UFPA, no IFUSP, etc. Ao Ivens Martins Carneiro, que mesmo sendo acima da média, sempre teve a humildade no seu jeito de ajudar e ensinar, em qualquer assunto, física, filosofia, matemática, linux, etc..., é isso! Obrigado por ter me dado a oportunidade de 
ser teu amigo e compartilhar todos aqueles momentos (do PET, UFPA e IFUSP) que não voltam, mas que recordo constantemente. Agradeço a ti "Vibens", por a isso tudo que proporcionastes a mim. Sinto muitas saudades...

À minha família todo amor que recebi durante todos esse anos, em especial, Tia Silvia e Tia Ângela, que me tratam como filho.

À meus irmãos Andressa e Alexandre, pela amizade, amor e pela infância maravilhosa que tivemos, e ao meus Pais Manoel e Ana Lúcia por todo amor, confiança, amizade, ensinamentos, que moldaram minha personalidade.

À minha esposa Rafaela por todo apoio, amor, compreensão e pela filha maravilhosa que temos. E a minha filha Maria Eduarda que veio sem avisar, mas que mudou meu jeito de ver o mundo com aquele sorriso lindo e pureza no olhar, sendo ela o melhor resultado que obtive em minha vida.

À todos que fizeram parte de minha formação, de maneira silenciosa desempenhando suas funções, nos ajudando a realizar nossos sonhos e atingir nossos objetivos. Meus sinceros agradecimentos, a todos vocês, da secretaria (Dna. Olga, Paulo e Mônica), da limpeza, do RU, HU e da portaria (em especial ao Samuel e Campos) que permitiram que eu me sentisse seguro durante as madrugadas e finais de semana de trabalho. 
Dedico este trabalho:

A meus pais Manoel e Ana Lúcia, aos meus irmãos Andressa e Alexandre, e as minhas esposa e filha Rafaela e Maria Eduarda. 
...tudo indo bem como sempre, as vezes mais difícil, outras vezes mais difícil ainda, mas sempre bem, temos sempre que pensar o mais positivo possivel, apesar das dificuldades,...

Ivens Martins Carneiro. 


\section{Sumário}

1 Introdução 1

2 Introdução ao espaço não comutativo $\quad 6$

2.1 Histórico . . . . . . . . . . . . . . . . . . . . 6

2.2 Quantização de Weyl . . . . . . . . . . . . . . . . . . . . . 9

2.3 O produto estrela . . . . . . . . . . . . . . . . . . 12

3 Interação em espaços comutativos $\quad 15$

3.1 A estrutura da teoria . . . . . . . . . . . . . . . 15

3.1.1 Transformações de coordenadas . . . . . . . . . . . . . . . 16

3.2 Dinâmica . . . . . . . . . . . . . . . . . . . . . . . . . . . . . 18

3.2.1 Gravitação como uma teoria de campos . . . . . . . . . . . . . . . 20

3.2 .2 Propagador do Gráviton . . . . . . . . . . . . . . . . . . . 24

3.3 Ação para o campo escalar interagindo com o gráviton . . . . . . . . . 26

3.3.1 Método alternativo . . . . . . . . . . . . . . . . 28

4 Interação em espaços não comutativos 34

4.1 Os Problemas de uma gravitação não comutativa . . . . . . . . . . . . 34 
4.2 Transformação restrita de coordenadas

e gravidade unimodular . . . . . . . . . . . . . . . . . . . 36

4.3 Cálculo da ação em espaços não comutativos . . . . . . . . . . . . . . . . . 41

5 Considerações finais

A Breve revisão sobre teorias de gauge

A.1 Quantização e regras de Feynman . . . . . . . . . . . . . . . . . 56

A.2 Teoria de Yang-Mills em espaços não-comutativos . . . . . . . . . . . . 66

B Cálculo das constates do tensor $C^{(n)}$

B.1 Cálculo de $C^{(1)} \ldots \ldots \ldots \ldots \ldots \ldots \ldots \ldots$

B.2 Cálculo de $C^{(2)} \ldots \ldots \ldots \ldots \ldots$. . . . . . . . . . . . . . . . . . 71 


\section{Capítulo 1}

\section{Introdução}

A descrição das interações fundamentais da natureza em termos de teorias de campos tem como ponto de partida uma formulação em termos da ação clássica para esses campos. A natureza dos campos (escalar, vetorial ou tensorial) e a forma analítica da densidade de lagrangiana fornecem toda a informação para descrever a propagação e interação entre as partículas associadas a cada tipo de campo. Desse modo, as leis fundamentais ficam completamente determinadas a partir do conhecimento da ação.

Quando tratamos apenas da propagação e das interações entre partículas de spin e massa determinados, sem considerar a interação gravitacional, um dos requisitos básicos da ação é a invariância sob transformações de Lorentz. Ou seja, a ação deve ser um escalar de Lorentz. Essa importante restrição é suficiente para caracterizar a propagação das partículas, mas não fixa a forma das interações. De fato, podemos escrever uma infinidade de termos de interação que não violam a invariância de Lorentz.

Uma das mais importantes idéias introduzidas na física do século passado, foi o princípio geral segundo o qual as leis físicas mais fundamentais podem ser "descobertas" utilizando princípios de simetria, ou seja, impondo a invariância da ação sob determinados grupos de transformação. A idéia de que todas as interações da natureza são fundamentalmente descritas por teorias de campos possuindo invariância sob transformaçôes de gauge 
locais constitui um dos princípios mais importantes da física das partículas elementares. Antes que esse princípio se consolidasse da forma como o entendemos atualmente, um longo caminho foi percorrido. Weyl em 1918 introduziu o conceito de invariância de gauge $^{1}$ generalizando a Teoria da Relatividade Geral de modo a descrever a gravitação e o eletromagnetismo de maneira unificada [1]. Embora a idéia original de Weyl tenha falhado como uma teoria física ${ }^{2}$, esse foi um importantíssimo passo na direção do entendimento do significado da invariância de gauge e de suas implicações físicas. O passo seguinte foi dado por Fock em 1926 ao estabelecer a conexão entre a ambigüidade de escolha dos potenciais vetor e escalar na teoria de Maxwell e a transformação de fase local da função de onda do elétron. É importante ressaltar que a formulação do princípio de invariância local, da maneira como o entendemos hoje, está dada nas equações (5) (transformações dos potenciais) e (9) (transformação de fase local) do artigo de Fock em [2]. Logo depois, o próprio Weyl [3], inspirado também por idéias de London [4], consagrou o princípio de invariância de gauge no contexto fisicamente correto. Com essa interpretação, foi possível obter um entendimento das interações eletromagnéticas em termos de um princípio simples, bem como estabelecer a relação entre a conservação da carga elétrica e a invariância local (um exemplo da relação entre simetrias e leis de conservação que já era conhecido no caso das simetrias de espaço-tempo).

Ao mesmo tempo, ficava claro que outras interações estavam envolvidas nos fenômenos que ocorrem na escala sub-nuclear. Já por volta da década de 1950 as interações fortes haviam sido bastante estudadas do ponto de vista fenomenológico. Uma interessante questão, do ponto de vista teórico, era a possibilidade de descrever todas interações em termos de teorias possuindo as mesmas características de simplicidade do Eletromagne-

\footnotetext{
${ }^{1} \mathrm{O}$ termo "gauge", que pode ser traduzido como calibre,teve sua origem na idéia desenvolvida por Weyl, segundo a qual escalas de comprimentos e tempos seriam calibrados por fatores não integráveis $\mathrm{e}^{\frac{e}{\gamma} \int \mathrm{d} x_{\mu} A^{\mu}}$, onde $A^{\mu}$ seria identificado com o potencial eletromagnético. Assim, da mesma forma que a interação gravitacional pode ser descrita em termos da conexão entre referenciais locais, o eletromagnetismo seria descrito em termos da conexão entre escalas locais.

${ }^{2} \mathrm{~A}$ natureza quântica da matéria introduz uma escala natural, dada pelo comprimento de onda Compton de uma partícula de massa $m, \lambda_{C}=h / m c$, a qual não pode depender da posição. Por outro lado, a idéia de de invariância local de escala estaria em contradição com este fato.
} 
tismo de Maxwell e, especialmente, de sua versão quantizada, ou seja, a Eletrodinâmica Quântica (QED)

Yang e Mills, em 1954, aplicaram o princípio de invariância local de gauge às interações entre nucleons (prótons e nêutrons), impondo que a simetria sob transformações do spin isotópico fosse localmente realizada [5]. Ao contrário do que ocorre na QED, onde o potencial eletromagnético $A_{\mu}$ não possui carga e portanto não interage consigo mesmo (a QED é uma teoria linear), a construção de Yang-Mills leva naturalmente a equações não-lineares para os potenciais. Tais potenciais são denominados campos de gauge não abelianos. Uma importante característica destes campos vetoriais é a sua massa nula. Embora as interações entre prótons e nêutrons não pudessem ser descritas como sendo mediadas por partículas de massa nula ${ }^{3}$ a idéia de Yang-Mills tornou-se o protótipo para os avanços que vieram a ocorrer cerca de vinte anos depois com o desenvolvimento da Cromodinâmica Quântica (QCD), teoria de gauge que descreve as interações fortes em termos dos constituintes mais elementares dos nucleons, e da teoria unificada das interações eletrofracas. Pela segunda vez o princípio de invariância local teve que esperar mais um pouco para que seu conteúdo físico pudesse ser devidamente apreciado. De qualquer forma, já era claro que tanto a QED como a gravitação obedeciam a este princípio. Restava saber se as interações que se manifestam na escala microscópica também poderiam ser entendidas com base no mesmo princípio.

A idéia básica da simetria de gauge pode ser exemplificada da seguinte forma. Consideremos um sistema físico descrito por uma lagrangiana invariante sob transformações de um grupo de simetria contínuo $G$. Ao promovermos a simetria a uma simetria local, fazendo $G \rightarrow G(x)$, onde $x=x_{\mu}(\mu=0,1,2,3)$ são as coordenadas do espaço-tempo, somos obrigados a generalizar as derivadas usuais $\partial_{\mu}$, substituindo-as por derivadas covariantes $D_{\mu}=\partial_{\mu}-i A_{\mu}$. As grandezas $A_{\mu}$ são potenciais vetores possuindo componentes nos geradores da álgebra de Lie do grupo $G$ e se transformam de tal forma que $D_{\mu}$ se

\footnotetext{
${ }^{3} \mathrm{O}$ curto alcance das interações nucleares, mediadas por píons massivos, já era conhecido há bastante tempo. A massa destes mediadores havia sido prevista teoricamente por Yukawa em 1935 [6] e confirmada experimentalmente por Lattes, Occhialini e Powell em 1947 [7]. Yukawa e Powell foram laureados com o prêmio Nobel em 1949 e 1950, respectivamente
} 
transforme co-variantemente. Portanto, a simetria local nos força a introduzir o campo $A_{\mu}$ o que por sua vez determina a forma das interações entre $A_{\mu}$ e os campos de matéria, assim como suas auto-interações (estas, como veremos, surgem quando o grupo de simetria é não-abeliano). No caso das interações eletrofracas há um conjunto de potenciais $A_{\mu}$ que podem ser identificados com o fóton e os bósons vetoriais $Z^{0}, W^{ \pm}$, enquanto na QCD $A_{\mu}$ contém os campos dos glúons. A fim de ilustrar explicitamente a aplicação do princípio de gauge, apresentamos no apêndice A uma breve revisão sobre esse tópico.

O foco da presente dissertação é a investigação das interações entre o quantum do campo gravitacional (o gráviton) e as outras partículas elementares, no contexto da teoria de campos formulada no espaço-tempo não comutativo, ou seja, quando o produto local dos campos é substituido pelo produto Moyal (produto $\star$ ). Desta forma, queremos (precisamos) saber qual é a forma da ação. Ao investigarmos essa questão, saberemos também se é ou não consistente introduzir o produto $\star$ da forma como foi feita, por exemplo, em [8]. Com o intuito de tornar a análise simples e transparente, trataremos apenas do setor envolvendo os campos do gráviton e escalar (real) sem massa.

A fim de esboçar a idéia geral da nossa abordagem é conveniente antes lembrar alguns aspectos básicos da versão não-comutativa da QED (NCQED). Sabemos que essa teoria possui uma estrutura muito semelhante a da teoria não abeliana de gauge (ver apêndice A). Tendo em vista a analogia existente entre a teoria de gauge do campo eletromagnético $A_{\mu} \mathrm{e}$ a teoria de gauge do campo de gráviton $h_{\mu \nu}$ é tentador supor que a versão não comutativa das interações entre grávitons e escalares preserve a simetria básica de invariância sob transformações gerias de coordenadas. No entanto, não podemos saber a priori qual deve ser a forma da versão não comutativa da ação.

Há porém uma abordagem bastante adequada para essa investigação. Primeiramente levamos em conta que estamos tratando das interações envolvendo campos de massa e spin definidos (gráviton de spin 2 e massa zero e escalar de spin e massa zero). Uma tal formulação requer uma métrica de fundo de Minkowski tal como ocorre em uma expansão de campo gravitacional fraco. A ação procurada tem então uma forma geral 
tal que, em ordem zero, é dada pela ação do campo escalar livre. O termo seguinte, envolvendo a interação com o gráviton, é linear em $h_{\mu \nu}$, e assim por diante. Embora não saibamos a priori qual deva ser a versão não comutativa do termo linear em $h_{\mu \nu}$, podemos invocar um argumento de simetria semelhante ao que existe na versão comutativa. No caso comutativo, sabemos que o termo linear (bem como todos os subseqüentes) pode ser obtido utilizando a identidade de Ward que resulta da invariância sob transformações gerias de coordenadas. No entanto, não podemos supor que a invariância sob transformações gerias de coordenadas possa ser empregada também no caso não comutativo. Veremos, porém, que mesmo no caso não comutativo há ainda uma simetria residual cujo parâmetro infinitesimal é restrito pela condição de unimodularidade. Em princípio, esse procedimento pode ser estendido indefinidamente. Na prática, em cálculos perturbativos, uma dada ordem da expansão de campos fracos é suficiente para determinar completamente um dado processo.

No próximo capítulo faremos uma breve introdução aos conceitos básicos de teoria de campos formulada em espaços não comutativos [9]. No capítulo 3 revisaremos alguns conceitos de gravitação. No capítulo 4 trataremos da determinação da ação não comutativa seguindo o esboço descrito acima. Finalmente, no capítulo 5 apresentaremos algumas conclusões. Para não quebrar a continuidade da apresentação, acrescentamos apêndices.

Ao longo de todo o texto, utilizaremos o sistema de unidades $\hbar=c=k_{B}=1$ ( $k_{B}$ é a constante de Boltzmann). As coordenadas de espaço-tempo $x^{\mu}$ são rotuladas por índices gregos $(\mu, \nu, \alpha \cdots=0,1,2,3)$, com $x^{0}=t$, e as coordenadas espaciais $x^{i}$ por índices latinos $(i=1,2,3)$. Os sinais da métrica de Minkowski $\eta^{\mu \nu}$ são $(+,-,-,-)$. A métrica do espaço-tempo curvo é denotada por $g_{\mu \nu}$. 


\section{Capítulo 2}

\section{Introdução ao espaço não comutativo}

\subsection{Histórico}

A formulação de teorias de campos em espaços não comutativos é um tema investigado a algum tempo. Na verdade, a não comutatividade é um conceito fundamental para expressar relações de incerteza em mecânica quântica [10]. A idéia de que as coordenadas espaço-temporais possam também não comutar em escalas de distâncias muito pequenas foi formalizada pela primeira vez por Snyder em 1947 [11, 12 $]^{1}$. Na mecânica quântica a não comutatividade aplica-se aos observáveis posição e momento, de modo que trabalhamos com funções $f(x)$ definidas num espaço "comutativo", no qual as coordenadas $x^{i}$ satisfazem a seguinte relação de comutação,

$$
\left[x^{i}, x^{j}\right]=0 ; \quad i, j=1,2,3 .
$$

De modo que para expressar a relação de incerteza em mecânica quântica, o espaço de fase deixa de ser contínuo e passa a ser discreto. Desta forma, passamos de um espaço de fase clássico para um espaço de fase quântico ao substituirmos as variáveis canônicas posição e momento, $x^{i}$ e $p^{j}$, pelos operadores Hermitianos $\mathbf{x}^{i}$ e $\mathbf{p}^{j}$, que obedeçam a relação de

\footnotetext{
${ }^{1}$ Consta que essas idéias já haviam sido consideradas por Heisemberg em uma carta escrita para Pierls. A idéia teria se propagado até Snyder pela via Pierls $\rightarrow$ Pauli $\rightarrow$ Oppenheimer $\rightarrow$ Snyder.
} 
comutação de Heisenberg $\left[\mathbf{x}^{i}, \mathbf{p}^{j}\right]=i \hbar \delta^{i j}$. A constante $\hbar$ passa a definir a "área" mínima no espaço de fase, conhecida como célula de Planck. No limite de $\hbar \rightarrow 0$, retorna-se ao espaço de fase clássico.

Um outro tipo de discretização pode ser aplicado sobre as coordenadas de posição, na qual as variáveis $x^{\mu}$ passam a ser mapeadas em operadores Hermitianos $\hat{x}^{\nu}$ de uma álgebra não comutativa de funções definida em um espaço comutativo, de modo que as coordenadas do espaço-tempo não comutem, satisfazendo a seguinte relação de comutação,

$$
\left[\hat{x}^{\mu}, \hat{x}^{\nu}\right]=i \theta^{\mu \nu}, \quad \mu, \nu=0,1,2,3
$$

na qual, o parâmetro $\theta$ é um tensor anti-simétrico com dimensão de comprimento ao quadrado $^{2}$. A não comutatividade das coordenadas introduz células mínimas de espaçotempo, nas quais há uma indeterminação dos respectivos observáveis. Assim, a interação pontual deixa de existir e as divergências ultravioletas talvez possam ser regularizadas por uma escala análoga à constante de Planck na mecânica quântica.

As implicações da relação (2.2) em uma teoria física são muito profundas e por vezes surpreendentes. Há várias razões para abordá-las, mas há também questões delicadas envolvidas, as quais fizeram com que até pouco tempo a teoria não fosse estudada mais seriamente. Talvez a principal razão para isso, é que ao postular-se uma relação de incerteza entre as medidas de posição levaria a uma teoria não local cujas propriedades não são bem conhecidas. No entanto, é possível postular a não-comutatividade das coordenadas espaço-temporais, mas apesar de muitas motivações físicas, a teoria de campos não comutativa não foi formulada por físicos e sim pelos matemáticos Connes e Riefel em 1987.

Uma das considerações em que a não comutatividade das coordenadas surge, procede de um argumento heurístico muito interessante [13]. Ao aplicarmos o princípio da incerteza de Heisemberg à gravitação clássica, teríamos uma medida das coordenadas em distâncias muito pequenas, assim teríamos uma indeterminação no momento perturbando o campo gravitacional nesta região.

\footnotetext{
${ }^{2} \mathrm{~A}$ álgebra de operadores foi extensivamente estudada, sendo conhecidas muitas propriedades.
} 
Uma leitura das Equações de Einstein, ver equação (3.15), nos mostra que devido a uma indeterminação no momento, uma energia seria transmitida ao sistema por meio do tensor energia-momento. Isto por sua vez, afetaria o campo gravitacional. Mais claramente, uma medida de posição em uma região a no espaço gera uma indeterminação de ordem 1/a no espaço dos momentos. Temos então pela proporcionalidade entre os campos gravitacional e a indeterminação no espaço dos momentos, que, quanto maior a precisão da medida nas coordenadas, maior a perturbação do campo gravitacional no ponto de medida, ou seja, maior será o campo gravitacional gerado pelo processo de medida. A localização deixará de ter um significado operacional quando a intensidade do campo gravitacional for suficientemente grande a ponto de impedir que qualquer sinal saia da região de localização. Tal efeito surgiria em medidas na ordem da escala de Planck ${ }^{3}$, logo a natureza quântica do campo gravitacional possivelmente deverá ser levada em conta neste cenário. Este argumento reforça a suspeita de que a teoria quântica da gravitação traria consigo a modificação da geometria na escala de Planck de modo a caminhar para uma descrição não local.

Historicamente, em teoria quântica de campos, a estrutura de não comutatividade para as coordenadas de espaço-tempo surgiu no final da década de 1940 [11, 12]. Neste contexto inicial, a idéia era utilizar a não comutatividade em escalas de comprimento muito pequenas para introduzir um corte na região de altos momentos como meio de controlar as divergências ultravioletas na eletrodinâmica quântica que até então não tinham solução. Devido ao sucesso obtido pelo procedimento de renormalização realizado na época, a abordagem foi logo esquecida.

Nos últimos anos o interesse em teorias não comutativas foi renovado quando se descobriu que a dinâmica da corda aberta na presença de um campo anti-simétrico pode ser descrita, em certos limites, em termos de teorias de calibre formuladas em espaços não comutativo [14]. O estudo dentro de um contexto mais amplo revelou que teorias não comutativas não são variações arbitrárias de teorias usuais de campos, e sim uma classe de teorias cujas propriedades merecem ser estudadas dentro de uma investigação de auto

\footnotetext{
${ }^{3}$ No que se refere à distâncias espaciais o comprimento é da ordem de $10^{-33} \mathrm{~cm}$.
} 
consistência.

Faremos nas próximas seções uma rápida apresentação dos conceitos básicos da teoria mais diretamente relacionados com o contexto do estudo desenvolvido nesta dissertação.

\subsection{Quantização de Weyl}

O formalismo que descreve a não comutatividade do espaço-tempo se inspira em algumas idéias fundamentais da mecânica quântica. Uma destas idéias, introduzida por Weyl, associava as variáveis $x^{i}$ definidas no espaço Euclidiano $\mathbb{R}^{D}$, a operadores $\hat{x}^{i}$ definido no espaço Euclidiano $\mathbb{R}_{\theta}^{D}$ não comutativo, em que, os operadores obedecem a relação $(2.2)$. Esta idéia, permite descrever o espaço não comutativo de maneira bastante geral e estudar sistematicamente as teorias de campos definidas no espaço Minkowski. Supondo que os campos desta teoria sejam representados por funções $f(x)$, satisfazendo as "condições

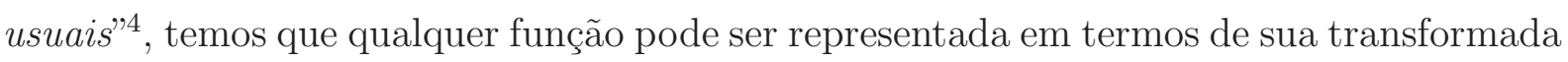
de Fourier

$$
\tilde{f}(k)=\int d^{4} x e^{-i k_{\mu} x^{\mu}} f(x) . \quad \quad \mu, \nu=0,1,2,3 .
$$

Assim, os operadores $\hat{x}^{\mu}$ são os geradores de uma álgebra não comutativa. De modo que a quantização de Weyl estabelece uma correspondência entre a álgebra dos campos em espaços comutativos e a álgebra dos operadores em espaços não comutativos. Dadas as componentes de Fourier de um campo qualquer, introduzimos o símbolo de Weyl por

$$
\hat{\mathcal{W}}[f]=\int \frac{d^{4} k}{(2 \pi)^{4}} \tilde{f}(k) \hat{T}(k, \hat{x}),
$$

no qual, o operador $\hat{T}(k, \hat{x})$ está representado por,

$$
\hat{T}(k, \hat{x})=e^{i k_{\mu} \hat{x}^{\mu}} .
$$

Como podemos perceber da definição (2.4), para cada $f(x)$ com $x$ pertencente ao espaço comutativo existe um $\hat{W}[f]$ no espaço não-comutativo.

\footnotetext{
${ }^{4}$ As derivadas de qualquer ordem se anulam no infinito tanto no espaço de posição quanto no espaço de momento [15].
} 
Podemos escrever o símbolo de Weyl de forma explicita através de um operador que chamaremos de $\hat{\Delta}(x)$, expresso por:

$$
\hat{\Delta}(x)=\int \frac{d^{4} k}{(2 \pi)^{4}} e^{i k_{\mu} \hat{x}^{\mu}} e^{-i k_{\mu} x^{\mu}}
$$

de forma que, o símbolo de Weyl expresso pela equação (2.4), poderá ser escrito por,

$$
\hat{\mathcal{W}}[f]=\int d^{4} x \tilde{f}(x) \hat{\Delta}(x) .
$$

Notamos que no caso comutativo quando $\theta^{\mu \nu}=0$ o operador (2.6) se reduz à função delta de Dirac $\delta^{4}(\hat{x}-x)$, de modo que $\hat{\Delta}(x)$ faz o mapeamento dos campos $f(x)$ com os operadores de Weyl.

É possível também mapear as derivadas usuais em "derivadas" de operadores definidas pelo operador anti-hermitiano $\hat{\partial}_{i}$ que são definidos pelas relações de comutações

$$
\left[\hat{\partial}_{\mu}, \hat{x}^{\nu}\right]=\delta_{\mu}^{\nu} \quad, \quad\left[\hat{\partial}_{\mu}, \hat{\partial}^{\nu}\right]=0
$$

Então de posse destas relações fica fácil mostrar que,

$$
\left[\hat{\partial}_{\mu}, \hat{\Delta}(x)\right]=-\partial_{\mu} \hat{\Delta}(x)
$$

de modo que ao substituirmos a equação (2.9) em (2.7), e realizando integrações por parte nesta, obtemos a relação

$$
\left[\hat{\partial}_{\mu}, \hat{\mathcal{W}}[f]\right]=\int d^{4} \partial_{\mu} f \hat{\Delta}(x)=\hat{\mathcal{W}}\left[\partial_{\mu} f\right]
$$

na qual, temos que o comutador do operador $\hat{\partial}_{i}$ com o símbolo de Weyl $\hat{\mathcal{W}}[f]$ atua como uma derivada usual no campo $f(x)$.

Introduzindo agora a operação de traço, denotada por Tr, segue de (2.7) que o traço do símbolo de Weyl será representado pela seguinte expressão,

$$
\operatorname{Tr} \hat{\mathcal{W}}[f]=\int d^{4} f(x) \operatorname{Tr} \hat{\Delta}(x) .
$$

Escolhendo a normalização $\operatorname{Tr} \hat{\Delta}(x)=1$, segue de (2.11) que

$$
\operatorname{Tr} \hat{\mathcal{W}}[f]=\int d^{4} f(x)
$$


De forma que o traço dos operadores de Weyl é representado por uma integração dos campos do espaço-tempo.

Assim, a relação (2.7) nos dá diretamente os operadores de Weyl a partir dos campos, ou seja, $f(x) \stackrel{\hat{\Delta}(x)}{\longmapsto} \hat{\mathcal{W}}[f]$, evidenciando ainda mais que (2.6) faz o mapeamento dos campos $f(x)$, no espaço comutativo, com os operadores de Weyl $\hat{\mathcal{W}}[f]$ no novo espaço não comutativo.

Agora que sabemos que $\hat{\Delta}(x)$ relaciona os elementos do espaço comutativo com os elementos do espaço não-comutativo, devemos discutir a multiplicação entre dois elementos $\hat{\mathcal{W}}[f]$ e $\hat{\mathcal{W}}[g]$. Como veremos na próxima seção, desta relação iremos naturalmente definir o produto Grönewold - Moyal entre duas funções $f(x)$ e $g(x)$ (também conhecido como produto estrela). Mas para isso, deve-se conhecer o produto de dois operadores (2.6) em pontos quaisquer do espaço-tempo. Usando esta equação e a fórmula de Baker-CampbellHausdorff,

$$
e^{\hat{A}+\hat{B}}=e^{\frac{1}{2}[\hat{A}, \hat{B}]} e^{\hat{A}+\hat{B}}
$$

podemos escrever o produto das exponenciais abaixo usando (2.2) como,

$$
e^{i k_{\mu} \hat{x}^{\mu}} e^{i k_{\nu}^{\prime} \hat{x}^{\nu}}=e^{\frac{1}{2}\left[i k_{\mu} \hat{x}, i k_{\nu}^{\prime} \hat{x}^{\nu}\right]} e^{i\left(k_{\mu}+k_{\mu}^{\prime}\right) \hat{x}^{\mu}}=e^{-\frac{i}{2} \theta^{\mu \nu} k_{\mu} k_{\nu}^{\prime}} e^{i k_{\mu} \hat{x}^{\mu}+i k_{\nu}^{\prime} \hat{x}^{\nu}}
$$

de modo que teremos

$$
\begin{aligned}
\hat{\Delta}(x) \hat{\Delta}(y) & =\iint \frac{d^{4} k}{(2 \pi)^{4}} \frac{d^{4} k^{\prime}}{(2 \pi)^{4}} e^{-\frac{i}{2} \theta^{\mu \nu} k_{\mu} k_{\nu}^{\prime}} e^{i k_{\mu} \hat{x}^{\mu}+i k_{\nu}^{\prime} \hat{x}^{\nu}} e^{-i k_{\mu} x^{\nu}-i k_{\mu}^{\prime} x^{\nu}} \\
& =\iint \frac{d^{4} k}{(2 \pi)^{4}} \frac{d^{4} k^{\prime}}{(2 \pi)^{4}} \int d^{4} z e^{i\left(k+k^{\prime}\right)_{\mu} z^{\mu}} \hat{\Delta}(z) e^{-\frac{i}{2} \theta^{\mu \nu} k_{\mu} k_{\nu}^{\prime}} e^{i k_{\mu} \hat{x}^{\mu}+i k_{\nu}^{\prime} \hat{x}^{\nu}}
\end{aligned}
$$

Calculando as integrais gaussianas em $k$ e $k^{\prime}$ (estamos assumindo que a matriz $\theta$ possui inversa), teremos

$$
\hat{\Delta}(x) \hat{\Delta}(y)=\frac{1}{\pi^{4}|\operatorname{det} \theta|} \int d^{4} z \hat{\Delta}(z) e^{-2 i\left(\theta^{-1}\right)_{\mu \nu}(x-z)^{\mu}(y-z)^{\nu}} .
$$

Usando a normalização do traço de $\Delta$ e a anti-simetria de $\theta^{-1}$, obtemos a seguinte condição de ortonormalidade

$$
\operatorname{Tr}(\hat{\Delta}(x) \hat{\Delta}(y))=\delta^{4}(x-y)
$$


Multiplicando o lado esquerdo de $(2.7)$ por $\hat{\Delta}(y)$, tomando o traço e usando a relação acima, obtemos a seguinte relação,

$$
f(x)=\operatorname{Tr}(\hat{\mathcal{W}}[f] \hat{\Delta}(y))
$$

na qual, a função $f(x)$ assim obtida a partir do correspondente operador quântico é denominada função de distribuição de Wigner e o mapeamento realizado por $\hat{\Delta}(x)$ é o análogo da correspondência da teoria quântica usual. Desta forma, a relação (2.16) irá contribuir para obtermos a representação entre os dois elementos $\hat{\mathcal{W}}[f]$ e $\hat{\mathcal{W}}[g]$.

\subsection{O produto estrela}

Conforme vimos na seção anterior, estabelecemos uma relação entre os elementos do espaço comutativo e o espaço não comutativo. Agora iremos verificar uma propriedade muito importante que é o produto dos operadores de Weyl $\hat{\mathcal{W}}[f]$ e $\hat{\mathcal{W}}[g]$. Uma tal expressão constitui o ponto de partida para que possamos mapear os operadores de Wigner de uma teoria de campos qualquer. Por exemplo, o produto de dois operadores possui a seguinte expressão no espaço de coordenadas

$$
\operatorname{Tr}(\hat{\mathcal{W}}[f] \hat{\mathcal{W}}[g] \hat{\Delta}(x))=\frac{1}{\pi^{4}|\operatorname{det} \theta|} \iint d^{4} y d^{4} z f(y) g(z) e^{-2 i\left(\theta^{-1}\right)_{\mu \nu}(x-y)^{\mu}(x-z)^{\nu}}
$$

Usando a expressão (2.4) no produto entre $\hat{\mathcal{W}}[f]$ e $\hat{\mathcal{W}}[g]$, substituindo as componentes de Fourier por (2.3) e levando em conta a fórmula de Baker-Campbell-Hausdorff, temos

$$
\hat{\mathcal{W}}[f] \hat{\mathcal{W}}[g]=\hat{\mathcal{W}}[f \star g]
$$

na qual, 


$$
\begin{aligned}
f(x) \star g(x) & =\iint \frac{d^{4} k}{(2 \pi)^{4}} \frac{d^{4} k^{\prime}}{(2 \pi)^{4}} \tilde{f}(k) \tilde{g}\left(k^{\prime}-k\right) e^{-\frac{i}{2} \theta^{\mu \nu} k_{\mu} k_{\nu}^{\prime}} e^{i k_{\mu}^{\prime} x^{\mu}} \\
& =f(x) e^{\frac{i}{2} \partial_{\mu} \theta^{\mu \nu} \partial_{\nu}} g(x) \\
& =f(x) g(x) \\
& +\sum_{n=1}\left(\frac{i}{2}\right)^{n}\left[\frac{1}{n !} \theta^{\mu_{1} \nu_{1}} \cdots \theta^{\mu_{n} \nu_{n}} \partial_{\mu_{1}} \cdots \partial_{\mu_{n}} f(x) \partial_{\nu_{1}} \cdots \partial_{\nu_{n}} g(x)\right]
\end{aligned}
$$

é o produto de Grönenwold-Moyal [16, 17]. É interessante considerar algumas conseqüências simples da expressão (2.20). Por exemplo usando a anti-simetria de $\theta^{\mu \nu}$, o comutador e o anti-comutador de Grönenwold-Moyal podem ser escritos como

$$
\begin{aligned}
{[f(x), g(x)]_{\star} } & \equiv f(x) \star g(x)-g(x) \star f(x) \\
& =2 i f(x) \operatorname{sen}\left(\frac{1}{2} \partial_{\mu} \theta^{\mu \nu} \partial_{\nu}\right) g(x)
\end{aligned}
$$

e

$$
\begin{aligned}
\{f(x), g(x)\}_{\star} & \equiv f(x) \star g(x)+g(x) \star f(x) \\
& =2 f(x) \cos \left(\frac{1}{2} \partial_{\mu} \theta^{\mu \nu} \partial_{\nu}\right) g(x)
\end{aligned}
$$

No caso geral, em que tivermos vários operadores, somos levados a considerar a seguinte extensão da fórmula (2.20)

$$
f_{1}\left(x_{1}\right) \star \cdots \star f_{n}\left(x_{n}\right)=\prod_{a<b} e^{\left(\frac{i}{2} \theta^{\mu \nu} \frac{\partial}{\partial x_{a}^{\mu}} \frac{\partial}{\partial x_{b}^{\nu}}\right)} f_{1}\left(x_{1}\right) \cdots f_{n}\left(x_{n}\right) .
$$

Assim temos que tal produto constitui uma alternativa ao produto de operadores de Weyl. A não comutatividade se manifesta como uma "deformação" do produto de funções das coordenadas do espaço-tempo. No caso do produto de vários operadores, temos que integrando este produto em todo espaço-tempo e usando a equação (2.11) obtemos a seguinte relação entre as duas maneiras de representar a não-comutatividade.

$$
\operatorname{Tr}\left(\hat{\mathcal{W}}\left[f_{1}\right] \cdots \hat{\mathcal{W}}\left[f_{n}\right]\right)=\int d^{4} x f_{1}(x) \star \cdots \star f_{n}(x)
$$


Levando em conta a propriedade de ciclicidade do traço, concluímos que a integral de um produto Grönenwold-Moyal permanece invariante quando permutamos ciclicamente as funções $f_{a}(x)$. E no caso especial de duas funções teremos

$$
\int d^{4} x f(x) \star g(x)=\int d^{4} x g(x) \star f(x)=\int d^{4} x f(x) g(x)
$$

Esta igualdade pode ser verificada diretamente usando a equação (2.20) e notando que os termos envolvendo $\theta$ se anulam ao serem integrados por partes. Vimos que a integração no espaço de coordenadas é formalmente uma operação de "traço" do produto deformado de funções. Estas propriedades serão muito úteis quando estivermos tratando abordagem não-comutativa no contexto das interações gravitacionais. 


\section{Capítulo 3}

\section{Interação entre grávitons e campos escalares em espaços comutativos}

Antes de pensarmos em uma teoria de gravitação em espaços não-comutativos, é necessário fazermos uma revisão sobre a teoria da relatividade. Esta revisão nos ajudará a entender a notação adotada, e será relevante para o entendimento dos tópicos subseqüentes.

\subsection{A estrutura da teoria}

O Princípio da Relatividade Especial, afirma que as leis da natureza são invariantes sob um grupo particular de transformações das coordenadas do espaço-tempo, chamado transformações de Lorentz. Esta afirmação foi feita por Einstein, já que no início do século $X X$ havia um conflito nas transformações de Galileu ${ }^{1}$, uma vez que as equações de Maxwell não são invariantes sobre tais transformações. E para resolver este conflito, Einstein modificou as leis de movimento de modo que estas fossem invariantes de Lorentz. Vejamos rapidamente como se deu esta modificação.

\footnotetext{
${ }^{1}$ Estas transformações relacionavam dois sistemas de coordenadas espaciais distintos, com o tempo absoluto, graças ao movimento de translação uniforme na direção de um dos eixos.
} 


\subsubsection{Transformações de coordenadas}

Uma transformação de Lorentz é dada pela mudança de um sistema de coordenadas do espaço-tempo $x^{\alpha}$ em um outro sistema $x^{\prime \alpha}$ e expressa por,

$$
x^{\prime \alpha}=\Lambda_{\beta}^{\alpha} x^{\beta}
$$

com a condição,

$$
\eta_{\mu \nu}=\Lambda_{\mu}^{\alpha} \Lambda_{\nu}^{\beta} \eta_{\alpha \beta}
$$

em que, $\eta_{\mu \nu}=(1,-1,-1,-1)$ é uma matriz diagonal, chamada métrica de Minkowski. No entanto, as transformações de Lorentz, refletem a invariância por transformações globais, e a propriedade fundamental que caracteriza esta transformação, é a invariância do intervalo de espaço-tempo de Minkowski, definido por,

$$
\mathrm{d} s^{2}=\eta_{\mu \nu} \mathrm{d} x^{\prime \mu} \mathrm{d} x^{\prime \nu}=\eta_{\alpha \beta} \mathrm{d} x^{\alpha} \mathrm{d} x^{\beta},
$$

em que, este resultado é a explicação teórica para o experimento de Michelson e Morley, no qual, a velocidade da luz, é a mesma em todas as direções analisadas, ou seja, em todos os sistemas inerciais.

No entanto, há um problema quando uma teoria é invariante apenas globalmente. Sabemos que a invariância devido as transformações de Lorentz constituem a base para o princípio da relatividade especial, que está caracterizada pelo movimento uniforme entre os dois observadores. A principal preocupação de Einstein era estender o princípio da relatividade para todos os observadores, pois seu objetivo era lidar com duas questões, a gravidade e os observadores acelerados. Por este motivo, Einstein sentia uma forte necessidade de generalizar o princípio da relatividade dos movimentos uniformes aos movimentos arbitrários. Em 1907, ao preparar um artigo de revisão encomendado por Johannes Stark, tem a idéia brilhante de analisar (localmente) o campo gravitacional, no referencial de um observador em queda livre. Para fazer isso, Einstein formulou o princípio da equivalência que lhe permitiu generalizar a relatividade dos movimentos uniformes aos movimentos acelerados. O princípio da equivalência afirma que em "cada ponto do espaçotempo localizado em um campo gravitacional arbitrário é possível escolher um sistema de 
coordenadas, tal que, dentro de uma região suficientemente pequena nas proximidades do ponto em questão, as leis da natureza possuem a mesma forma como se estivesse em um sistema cartesiano de coordenadas sem aceleração, na ausência da gravidade"[18].

Para verificarmos esta afirmação, usamos um método que é uma versão alternativa ao princípio da equivalência conhecido como princípio da covariância geral, este método afirma que uma equação física é válida na ausência de um campo gravitacional, se ela concorda com as leis da relatividade especial, quando o tensor métrico $g_{\mu \nu}$ é igual ao tensor métrico de Minkowski $\eta_{\mu \nu}$, e quando ela preserva sua forma quando realizamos uma transformação geral de coordenadas $x \rightarrow x^{\prime}$.

O método da covariância geral, foi utilizado por Einstein para escrever equações físicas que são invariantes por uma transformação geral de coordenadas. Desta forma, na ausência de um campo gravitacional, o tensor métrico é definido em um sistema geral de coordenadas $x^{\mu}$ por

$$
g_{\mu \nu} \equiv \eta_{\alpha \beta} \frac{\partial \xi^{\alpha}}{\partial x^{\mu}} \frac{\partial \xi^{\beta}}{\partial x^{\nu}}
$$

em que, $\xi^{\alpha}$ é a coordenada de um sistema localmente inercial. Tomando (3.4) em um sistema de coordenadas diferente expresso por $x^{\prime \mu}$, o tensor métrico será,

$$
g^{\prime}{ }_{\mu \nu} \equiv \eta_{\alpha \beta} \frac{\partial \xi^{\alpha}}{\partial x^{\prime \mu}} \frac{\partial \xi^{\beta}}{\partial x^{\prime \nu}}=\eta_{\alpha \beta} \frac{\partial \xi^{\alpha}}{\partial x^{\rho}} \frac{\partial x^{\rho}}{\partial x^{\prime \mu}} \frac{\partial \xi^{\beta}}{\partial x^{\sigma}} \frac{\partial x^{\sigma}}{\partial x^{\prime \nu}} .
$$

De acordo com (3.4) temos que o tensor métrico escrito em termos de uma transformação geral de coordenadas é dado por,

$$
g_{\mu \nu}^{\prime}=g_{\rho \sigma} \frac{\partial x^{\rho}}{\partial x^{\prime \mu}} \frac{\partial x^{\sigma}}{\partial x^{\prime \nu}} .
$$

O tensor $g_{\mu \nu}$ é conhecido como tensor métrico covariante e seu inverso é um tensor contravariante definido por $g^{\gamma \mu}$, de modo que,

$$
g^{\gamma \mu} g_{\mu \nu}=\delta_{\nu}^{\gamma}
$$

Além da invariância das equações físicas, o princípio da covariância geral, permitenos construir um tensor que é formado pelo tensor métrico e suas primeiras e segundas 
derivadas, este tensor é conhecido como tensor de curvatura e é definido por,

$$
R_{\mu \nu \kappa}^{\lambda}=\partial_{\kappa} \Gamma_{\mu \nu}^{\lambda}-\partial_{\nu} \Gamma_{\mu \kappa}^{\lambda}+\Gamma_{\mu \nu}^{\tau} \Gamma_{\kappa \tau}^{\lambda}-\Gamma_{\mu \kappa}^{\tau} \Gamma_{\nu \tau}^{\lambda}
$$

em sua forma contraída é conhecido como tensor de Ricci

$$
R_{\mu \nu}=R_{\mu \lambda \nu}^{\lambda}
$$

sendo a curvatura escalar expressa por,

$$
R=g^{\mu \nu} R_{\mu \nu}
$$

O símbolo de Christoffel $\Gamma_{\mu \nu}^{\lambda}$ é dado em termos das derivadas do tensor métrico.

$$
\Gamma_{\mu \nu}^{\lambda}=\frac{1}{2} g^{\lambda \rho}\left(\partial_{\mu} g_{\rho \nu}+\partial_{\nu} g_{\rho \mu}-\partial_{\rho} g_{\mu \nu}\right)
$$

A existência do tensor de curvatura, reafirma a questão de que o princípio da equivalência juntamente com o princípio da covariância geral determinam unicamente os efeitos da gravitação em sistemas arbitrários.

A utilização destas propriedades do tensor métrico nos ajudarão a construir uma teoria de interação gravitacional. No entanto, estudaremos o caso mais simples que é a interação do gráviton com o campo escalar.

\subsection{Dinâmica}

A ação que descreve a dinâmica da métrica é,

$$
S=\bar{M}_{p}^{2} \int d^{4} x \sqrt{-g} R ; \quad \bar{M}_{p}^{2}=\frac{1}{16 \pi G}
$$

na qual, $g$ é o determinante do tensor métrico $g_{\mu \nu}$ do espaço-tempo. A constante $G$ é a constante de Newton, que possui dimensão de inverso da massa ao quadrado, e $\bar{M}_{p}$ é a massa de Planck. A dependência explícita na métrica $g_{\mu \nu}$ está encapsulada nas relações (3.8). 
É interessante destacar, no presente contexto, as seguintes característica da ação de Einstein-Hilbert. Como vimos na seção anterior a primeira delas é a evidente não linearidade, ou seja, a ação contém termos envolvendo o produto de mais de dois tensores $g_{\mu \nu}$. Uma outra propriedade evidente é que todos os termos da ação possuem exatamente duas potências da derivada $\partial$, como pode ser verificado diretamente nas relações acima. Por último, o mais importante, é que a forma desta ação é determinada pela invariância sob transformações gerais de coordenadas do tipo

$$
x^{\mu} \rightarrow x^{\prime \mu}=x^{\mu}-w^{\mu}(x) .
$$

Embora a gravitação tenha sido originalmente desenvolvida por Einstein em termos estritamente geométricos, é também possível adotar um ponto de vista onde as interações gravitacionais são descritas, de maneira análoga às outras interações conhecidas, pela troca dos quanta de um campo gravitacional, ou seja, grávitons.

Antes de prosseguir, é necessário também entender de que maneira são descritas as interações pela troca de grávitons. O procedimento mais simples e natural consiste em modificar a ação da teoria de campos, originalmente formulada no espaço de Minkowski e invariante de Lorentz, de modo a torná-la invariante sob as transformações (3.11). Esta condição será satisfeita se adotarmos as seguintes prescrições mínimas

$$
\begin{aligned}
\int d^{4} x & \longrightarrow \int d^{4} x \sqrt{-g} \\
\eta^{\mu \nu} & \longrightarrow g^{\mu \nu} \\
\partial_{\mu} & \longrightarrow D_{\mu}
\end{aligned}
$$

na qual, $D_{\mu}$ é a derivada co-variante da relatividade geral satisfazendo

$$
\begin{gathered}
D_{\mu} \phi=\partial_{\mu} \phi \\
D_{\nu} A_{\mu}=\partial_{\nu} A_{\mu}-\Gamma_{\mu \nu}^{\alpha} A_{\alpha}, \\
D_{\sigma} A_{\mu \nu}=\partial_{\sigma} A_{\mu \nu}-\Gamma_{\mu \sigma}^{\alpha} A_{\alpha \nu}-\Gamma_{\nu \sigma}^{\alpha} A_{\mu \alpha}, \\
D_{\sigma} A_{\mu \nu \ldots}=\partial_{\sigma} A_{\mu \nu \ldots}-\text { um } \Gamma \text { para cada índice. }
\end{gathered}
$$


Seguindo as prescrições acima, temos que a ação para a teoria de campos escalares em interação com campos gravitacionais é dada por,

$$
S_{\text {scal. }}[\phi, g]=\frac{1}{2} \int d^{4} x \sqrt{-g}\left(g^{\mu \nu} \partial_{\mu} \phi \partial_{\nu} \phi-m^{2} \phi\right) .
$$

As equações de movimento clássicas são obtidas usando o princípio variacional na ação total $^{2}$. Fazendo isso, via formalismo de Euler-Lagrange, obtemos a equação de Einstein

$$
R_{\mu \nu}-\frac{1}{2} R g_{\mu \nu}=-8 \pi G T_{\mu \nu}
$$

na qual, $T_{\mu \nu}$ é o tensor de energia-momento associado aos diversos campos de natureza não gravitacional ( $T^{\mu 0}$ e $T^{\mu i}$ são respectivamente a densidade e o fluxo de energia-momento).

\subsubsection{Gravitação como uma teoria de campos}

Vamos agora introduzir o contexto físico no qual a noção do gráviton, mencionada anteriormente, emerge naturalmente. Nosso ponto de partida será um dos resultados mais profundos da teoria de gravitação, sintetizado na relação

$$
T^{\mu \nu}=-\frac{2}{\sqrt{-g}} \frac{\delta S_{M}}{\delta g_{\mu \nu}} .
$$

Esta equação nos informa que o tensor de energia-momento $T^{\mu \nu}$ pode ser obtido considerando a ação $S_{M}$, de quaisquer outros campos, formulada no espaço de métrica $g_{\mu \nu}$ (seguindo as prescrições dadas na seção anterior) e tomando a variação em relação à $g_{\mu \nu}$, mantendo $x^{\mu}$ fixo. De fato, introduzindo o campo $h_{\mu \nu}$ tal que ${ }^{3}$

$$
g_{\mu \nu}=\eta_{\mu \nu}+h_{\mu \nu}
$$

expandindo a ação $S_{M}$ até primeira ordem em $h_{\mu \nu}$, e levando em conta (3.16), teremos

$$
S_{M}=S_{M}(h=0)-\int d^{4} x\left(\frac{1}{2} h_{\mu \nu} T^{\mu \nu}+\mathcal{O}\left(h^{2}\right)\right),
$$

\footnotetext{
${ }^{2} \mathrm{~A}$ ação total é escrita da seguinte forma, $S_{T}=S+S_{\text {scal }}$.

${ }^{3}$ Estamos absorvendo um fator $\sqrt{32 \pi G}$ em $h_{\mu \nu}$. Portanto, $h_{\mu \nu}$ é admensional
} 
ou seja, o efeito da gravitação é "sentido" por todos os outros campos através do acoplamento de $h_{\mu \nu}$ com o tensor energia-momento no espaço plano. Do ponto de vista físico este é um bom motivo para se atribuir a característica de campo do gráviton ao tensor $h_{\mu \nu}$. Temos assim um cenário no qual a interação gravitacional ocorre no espaço plano de Minkowski, analogamente à interação do campo do fóton $A_{\mu}$ com a corrente eletromagnética $J^{\mu}$.

É interessante notar que esta abordagem permite também obter certas propriedades gerais do tensor de energia-momento. De fato, considerando a forma da ação

$$
S_{M}=\int d^{4} x \sqrt{-g}\left(A+g^{\mu \nu} B_{\mu \nu}+g^{\mu \nu} g^{\lambda \rho} C_{\mu \nu \lambda \rho}+\cdots\right)
$$

expandindo a métrica até ordem em $h_{\mu \nu}$ (mais detalhes na próxima seção)

$$
\sqrt{-g}=1+\eta^{\mu \nu} h_{\mu \nu}+\mathcal{O}\left(h^{2}\right) ; \quad g^{\mu \nu}=\eta^{\mu \nu}-h^{\mu \nu}+\mathcal{O}\left(h^{2}\right)
$$

e comparando com (3.18), obtemos a seguinte expressão geral para o tensor de energiamomento no espaço plano

$$
T_{\mu \nu}=2\left(B_{\mu \nu}+2 C_{\mu \nu \lambda \rho} \eta^{\lambda \rho}+\cdots\right)-\eta_{\mu \nu} \mathcal{L}
$$

na qual, $\mathcal{L}$ é a lagrangiana no espaço de Minkowski. Tomando o traço

$$
\eta^{\mu \nu} T_{\mu \nu}=-4 A-2 \eta^{\mu \nu} B_{\mu \nu}
$$

Vemos que não há uma contribuição do termo $g^{\mu \nu} g^{\lambda \rho} C_{\mu \nu \lambda \rho}$ para o traço do tensor energiamomento. É precisamente este tipo de termo que seria gerado se aplicarmos as prescrições (3.12) à lagrangiana de Maxwell. Portanto, o tensor de energia momento do campo eletromagnético possui traço nulo.

Substituindo (3.17) na ação gravitacional (3.10), vamos obter uma ação para o campo do gráviton dada por uma soma de infinitos termos em ordem crescente de "potências" de $h_{\mu \nu}$, iniciando pelo termo quadrático. Como vimos anteriormente, cada termo tem duas derivadas $\partial$. Esquematicamente podemos escrever

$$
S=\bar{M}_{p}^{2} \int d^{4} x \sqrt{g}\left(\partial h \partial h+h \partial h \partial h+h^{2} \partial h \partial h+\cdots\right) .
$$


Cada um dos termos acima representa, na verdade, uma combinação das diversas possíveis contrações dos campos $h_{\mu \nu}$ e das derivadas $\partial_{\mu}$. Obviamente estas contrações são feitas com o tensor de Minkowski $\eta_{\mu \nu}$ e cada termo individual é um escalar de Lorentz. Deixando de lado as óbvias dificuldades de cálculo algébrico ${ }^{4}$, temos aqui um cenário conceitualmente idêntico ao das teorias de campos usuais nos quais a ação possui um termo quadrático e termos de interação. Os termos de interação em (3.23) descrevem o auto acoplamento do gráviton de maneira análoga aos termos de interação das teorias de Yang-Mills. Enquanto a auto-interação dos campos de Yang-Mills ocorre por que os campos carregam carga, no caso dos grávitons a auto-interação se dá porque o gráviton (como qualquer outra partícula) carrega energia.

A forma explícita de cada um dos termos em (3.23) pode ser obtida diretamente substituindo (3.17) em (3.8a) e (3.10) e expandindo a métrica, sua inversa e seu determinante até a ordem desejada. É possível também explorar uma interessante alternativa baseada em considerações de simetria, que além de tornar os cálculos muito mais simples, permite identificar o campo $h_{\mu \nu}$ como um autêntico campo de gauge, análogo ao campo de Yang-Mills. O ponto chave consiste em notar que a ação (3.23) é invariante sob as transformações (3.11), na qual a métrica transforma-se como

$$
\begin{aligned}
g_{\mu \nu}^{\prime}\left(x^{\prime}\right) & =\frac{\partial x^{\alpha}}{\partial x^{\prime \mu}} \frac{\partial x^{\beta}}{\partial x^{\prime \nu}} g_{\alpha \beta}(x) \\
& =\left(\delta_{\mu}^{\alpha}+\partial_{\mu} w^{\alpha}(x)\right)\left(\delta_{\nu}^{\beta}+\partial_{\nu} w^{\beta}(x)\right) g_{\alpha \beta}(x) \\
g_{\mu \nu}^{\prime}(x)-w^{\alpha}(x) \partial_{\alpha} g_{\mu \nu}^{\prime}(x) & =g_{\mu \nu}(x)+g_{\alpha \nu}(x) \partial_{\mu} w^{\alpha}(x)+g_{\alpha \mu}(x) \partial_{\nu} w^{\alpha}(x) \\
\delta g_{\mu \nu}(x) & =g_{\alpha \nu} \partial_{\mu} w^{\alpha}+g_{\alpha \mu} \partial_{\nu} w^{\alpha}+w^{\alpha} \partial_{\alpha} g_{\mu \nu}
\end{aligned}
$$

Na última linha, $\delta g_{\mu \nu}(x) \equiv g_{\mu \nu}^{\prime}-g_{\mu \nu}$ e $w^{\mu}$ são funções de $x^{\mu}$. Usando a equação $(3.13 \mathrm{~b})$ a última linha da equação acima pode ser escrita de maneira bem mais elegante como

$$
\delta g_{\mu \nu}=D_{\mu} w_{\nu}+D_{\nu} w_{\mu}
$$

Portanto, usando (3.17), vemos que o campo gravitacional se transforma como

$$
h^{\prime}{ }_{\mu \nu}-h_{\mu \nu} \equiv \delta h_{\mu \nu}=D_{\mu} w_{\nu}+D_{\nu} w_{\mu}
$$

\footnotetext{
${ }^{4}$ Tais dificuldades podem ser tratadas utilizando-se computação simbólica
} 
A semelhança ${ }^{5}$ formal entre a transformação de gauge para os campos de Yang-Mills é evidente. É importante ressaltar que a transformação (3.26) é linear em $h_{\mu \nu}$, como pode ser constatado substituindo (3.17) na última linha de (3.24). De forma que a gravitação formulada como uma teoria de campos revela sua invariância de gauge de maneira equivalente à teoria de Yang-Mills.

Agora, considerando separadamente cada termo da ação (3.23), é possível verificar que estes termos não são invariantes sob as transformações (3.24). No entanto, a invariância da ação faz com que um termo de ordem $h^{n}$ esteja relacionado com um termo de ordem $h^{n+1}$. Para verificar essas identidades começaremos pelo termo quadrático da ação, escrevendo todas as possíveis contrações compatíveis com a invariância de Lorentz e contendo duas derivadas. A ação mais geral que satisfaz este critério é ${ }^{6}$

$$
S^{0}[h]=\int \mathrm{d}^{4} x\left(a \partial_{\alpha} h^{\mu \nu} \partial^{\alpha} h_{\mu \nu}+b \partial_{\alpha} h_{\mu}^{\mu} \partial^{\alpha} h_{\nu}^{\nu}+c \partial_{\alpha} h^{\alpha \nu} \partial^{\mu} h_{\mu \nu}+d \partial^{\mu} h_{\alpha}^{\alpha} \partial^{\nu} h_{\mu \nu}\right)
$$

Impondo a condição de invariância $\delta S^{0}[h]=S^{0}\left[h^{\prime}\right]-S^{0}[h]=0$, com $h_{\mu \nu}^{\prime}$ dado por (3.26), e usando integração por partes para a contribuição linear em $h_{\mu \nu}$, teremos a seguinte condição ${ }^{7}$

$$
(4 a+2 c) \omega^{\nu} \partial^{2} \partial^{\mu} h_{\mu \nu}+(4 b+2 d) \omega^{\nu} \partial^{2} \partial^{\nu} h+(2 c+2 d) \omega^{\nu} \partial_{\nu} \partial^{\mu} \partial^{\lambda} h_{\mu \lambda}=0 .
$$

A existência de três estruturas independentes e quatro constantes a serem determinadas é uma excelente oportunidade de fazer contato com o limite newtoniano, fixando a normalização em termos da constante de Newton $G$. Isso requer uma normalização tal que, em

\footnotetext{
${ }^{5}$ Os campos vetoriais de gauge se transformam da seguinte forma,

$$
A_{\mu}^{a} \rightarrow A_{\mu}^{a}+D^{a b}{ }_{\mu} w^{b}
$$

em que,

$$
D^{a b}{ }_{\mu} \equiv \delta^{a b} \partial_{\mu}-f^{a b c} A^{c}{ }_{\mu} .
$$

Mais detalhes sobre a teoria de Yang-Mills, podem ser encontrados no apêndice A.

${ }^{6}$ Devemos considerar todas as contrações de pares de índices em $\partial^{\alpha_{1}} h^{\mu_{1} \nu_{1}} \partial^{\alpha_{2}} h^{\mu_{2} \nu_{2}}$. Há dois termos com $\alpha_{1}$ contraído com $\alpha_{2}$ e dois termos com $\alpha_{1}$ contraído com um dos índices de $h$. Esta sistemática pode ser generalizada para ordens superiores.

${ }^{7} \mathrm{O}$ termo quadrático em $h$ se combina com a variação da parte cúbica da ação produzindo as condições que determinam a ação em ordem $h^{3}$.
} 
unidades de $\bar{M}_{P}^{2}$, as constantes em (3.28) sejam escolhidas como $a=1, b=-1, c=-2$ e $d=2$. Substituindo estes valores em (3.27), teremos

$$
\begin{array}{r}
S^{0}[h]=\frac{\bar{M}_{P}^{2}}{2} \int \mathrm{d}^{4} x\left(\frac{1}{2} \partial_{\alpha} h^{\mu \nu} \partial^{\alpha} h_{\mu \nu}-\frac{1}{2} \partial_{\alpha} h_{\mu}^{\mu} \partial^{\alpha} h_{\nu}^{\nu}-\right. \\
\left.\partial_{\alpha} h^{\alpha \nu} \partial^{\mu} h_{\mu \nu}+\partial^{\mu} h_{\alpha}^{\alpha} \partial^{\nu} h_{\mu \nu}\right) .
\end{array}
$$

Como não poderia deixar de ser, se tivéssemos feito o cálculo direto, tedioso e não instrutivo, usando a expansão da métrica na equação (3.10), teríamos obtido o mesmo resultado acima.

Em princípio podemos prosseguir com este algoritmo, obtendo termos de ordem superior em $h$ como os indicados simbolicamente em (3.23).

\subsubsection{Propagador do Gráviton}

Por completeza, faremos uma breve digressão no sentido de determinar o propagador do gráviton. Desta forma, a obtenção das regras de Feynman para os vértices que descrevem as auto-interações do gráviton (vértice cúbico, quártico, etc) é um procedimento automatizável que não envolve qualquer tipo de dificuldade conceitual. Como já mencionamos, "qualquer computador", devidamente programado, pode fazer isso. Por isso, vamos focalizar aqui a obtenção do propagador do gráviton. Um texto clássico sobre este assunto é a referência [19], onde o ponto de partida é a construção do propagador de uma partícula de spin 2, levando em conta que as duas outras possibilidades mais simples, spin 0 ou spin 1, são descartadas por fatos experimentais elementares ${ }^{8}$.

A fim de exibir explicitamente o problema envolvido na obtenção do propagador do gráviton é conveniente usar integração por partes de modo a escrever (3.29) como

$$
S^{0}[h]=\frac{\bar{M}_{P}^{2}}{2} \int \mathrm{d}^{4} x \int \mathrm{d}^{4} x h_{\mu \nu} Q_{\text {transv. }}^{\mu \nu ; \alpha} h_{\alpha \beta},
$$

\footnotetext{
${ }^{8} \mathrm{O}$ campo gravitacional não pode ser escalar ( $\operatorname{spin} 0$ ) uma vez que a gravitação se acopla à energia, a qual não é uma grandeza escalar. Um campo vetorial (spin 1) também deve ser descartado, tendo em vista que partículas e anti-partículas, de qualquer tipo, interagem gravitacionalmente da mesma maneira, já que a gravitação é sempre atrativa.
} 
onde $Q_{\text {transv. }}^{\mu \nu ; \alpha \beta}$ atua no espaço linear de tensores (de Minkowski) simétricos de dois índices.

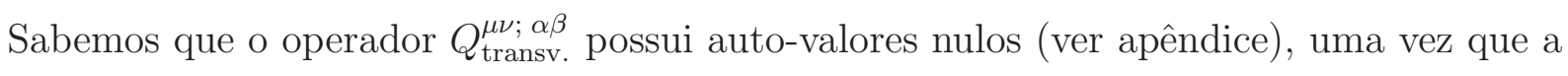
invariância de gauge, ver equação (3.26) implica em

$$
Q_{\text {transv. }}^{\mu \nu ; \alpha \beta} \partial_{\alpha} \omega_{\beta}=0
$$

Portanto, não é possível obter o operador inverso de $Q_{\text {transv. }}^{\mu \nu ; \alpha}$, ou seja, não podemos obter o propagador do gráviton. A solução para este problema é a mesma empregada no caso da QED, em que, fazemos uma escolha de gauge, impondo uma condição sobre os campos $h_{\mu \nu}$ de tal forma que a integração funcional seja feita somente sobre regiões não conectadas por transformações de gauge. Seguindo um procedimento análogo ao adotado para a teoria de Yang-Mills (ver apêndice A), introduzimos um termo extra à ação, o qual viola a invariância sob transformações de coordenadas, e modifica o termo quadrático de tal forma a torna-lo inversível. Uma escolha usual para esta fixação de gauge consiste em adicionar um termo tal que a equação de movimento resultante é a equação de onda $\partial^{2} h_{\mu \nu}=0$, no vácuo. Por isso esta escolha é as vezes denominada gauge harmônico. Esta escolha também resulta na forma mais simples para o propagador, analogamente ao caso do gauge de Feynman na QED. Para ver como isso pode ser feito, consideremos inicialmente a forma explícita do termo quadrático em (3.30) a qual pode ser escrita equivalentemente como ${ }^{9}$.

$$
\begin{aligned}
Q_{\text {transv. }}^{\mu \nu ; \alpha \beta} & =\frac{1}{4}\left[-K^{\mu \nu ; \alpha \beta} \partial^{2}+\eta^{\mu \nu} \eta^{\alpha \beta} \partial^{2}+\right. \\
& +\eta^{\nu \beta} \partial^{\mu} \partial^{\alpha}+\eta^{\mu \beta} \partial^{\nu} \partial^{\alpha}+\eta^{\nu \alpha} \partial^{\mu} \partial^{\beta}+\eta^{\mu \alpha} \partial^{\nu} \partial^{\beta} \\
& \left.-2 \eta^{\mu \nu} \partial^{\alpha} \partial^{\beta}-2 \eta^{\alpha \beta} \partial^{\mu} \partial^{\mu}\right],
\end{aligned}
$$

onde foi introduzida a grandeza

$$
K_{\mu \nu ; \alpha \beta} \equiv \frac{1}{2}\left(\eta_{\mu \alpha} \eta_{\nu \beta}+\eta_{\mu \beta} \eta_{\nu \alpha}-\eta_{\mu \nu} \eta_{\alpha \beta}\right)
$$

É fácil verificar que (aqui as contrações são efetuadas com a métrica de Minkowski $\eta_{\mu \nu}$ )

$$
K_{\mu \nu ; \rho \sigma} K^{\rho \sigma ; \alpha \beta}=\frac{1}{2}\left(\delta_{\mu}^{\alpha} \delta_{\nu}^{\beta}+\delta_{\mu}^{\beta} \delta_{\nu}^{\alpha}\right) \equiv I_{\mu \nu}^{\alpha \beta},
$$

\footnotetext{
${ }^{9}$ Esta forma é obtida usando integração por partes em (3.29), simetrizando nos pares de índices de cada campo e levando em conta a simetria bosônica $\mu \nu \leftrightarrow \alpha \beta$.
} 
onde $I^{\alpha \beta}{ }_{\mu \nu}$ é a matriz identidade no espaço de tensores simétricos de dois índices. A identidade (3.34) sugere que uma escolha de gauge conveniente é aquela que elimina todos os termos de (3.32), exceto o primeiro. Esse critério pode ser implementado adicionando à ação gravitacional o seguinte termo de fixação de gauge

$$
S_{\text {fix. }}^{0}[h]=\frac{\bar{M}_{P}^{2}}{2} \frac{1}{\xi} \int \mathrm{d}^{4} x f^{\mu}(h) f_{\mu}(h) \operatorname{com} f^{\mu}(h) \equiv \partial_{\alpha} h^{\alpha \mu}-\frac{1}{2} \partial^{\mu} h_{\lambda}^{\lambda}
$$

e fazendo $\xi=1^{10}$. Desse modo, o novo termo quadrático pode ser facilmente invertido, usando (3.34), levando à seguinte expressão para o propagador do gráviton no espaço de momento

$$
\begin{aligned}
& D_{\mu \nu \alpha \beta}(k)=\frac{1}{2 k^{2}}\left\{\eta_{\mu \alpha} \eta_{\nu \beta}+\eta_{\nu \alpha} \eta_{\mu \beta}-\eta_{\mu \nu} \eta_{\alpha \beta}\right. \\
\mu \nu & \alpha \beta
\end{aligned}
$$

Este é o análogo do propagador no gauge de Feynman na QED. Na gravitação este gauge é freqüentemente denominado gauge de Feynman-De Donder. Uma classe mais geral de gauges co-variantes (co-variância de Lorentz) é caracterizada por um parâmetro real $\xi$ qualquer.

Nesta dissertação não analisaremos os vértices de auto-interação.

\subsection{Ação para o campo escalar interagindo com o gráviton}

Nossos estudos estão sendo direcionados à investigação de uma ação não-comutativa, que represente as interações de campos escalares com o gráviton como foi explicitado na

\footnotetext{
${ }^{10}$ Note que este termo é de fato não invariante sob transformações gerais de coordenadas.
} 
seção anterior. No entanto, para estruturarmos nossa investigação é necessário primeiramente analisarmos o contexto usual de uma teoria comutativa de um campo escalar sem massa interagindo com grávitons. Para isso, consideraremos a ação (3.14), sem o termo de massa. Esse é o modelo mais simples envolvendo interações gravitacionais e que pretendemos generalizar para espaços não-comutativos, no próximo capítulo. Tomando $m=0$ na equação (3.14) teremos,

$$
S(\phi, g)=\frac{1}{2} \int d^{4} x \sqrt{-g}\left(g^{\mu \nu} \partial_{\mu} \phi \partial_{\nu} \phi\right)
$$

em que, a introdução do campo do gráviton $h_{\mu \nu}$ pode ser feita usando a expressão do tensor métrico $g_{\mu \nu}$, através da equação (3.17), como segue abaixo

$$
g_{\mu \nu} \equiv \eta_{\mu \nu}+\kappa h_{\mu \nu}
$$

em que, $\kappa \equiv \sqrt{32 \pi G}$. Usando a equação (3.7), calculamos as expressões em (3.20), fazendo a expansão em $\kappa$ para tensor métrico, assim como para $\sqrt{-g}$, de modo que teremos sua forma contravariante, dada por,

$$
g^{\mu \nu} \cong \eta^{\mu \nu}-\kappa h^{\mu \nu}+\kappa^{2} h^{\mu \alpha} h_{\alpha}^{\nu}+\cdots
$$

e $\sqrt{-g}$, como mostra o seguinte desenvolvimento,

$$
\begin{aligned}
g & =\operatorname{det}\left(g_{\mu \nu}\right) \\
& =\exp \left\{\operatorname{Tr}\left[\ln \left(\eta_{\mu \nu}+\kappa h_{\mu \nu}\right)\right]\right\} \\
& =\operatorname{det}\left(\eta_{\mu \alpha}\right) \exp \left\{\operatorname{Tr}\left[\ln \left(\delta^{\alpha}{ }_{\nu}+\kappa h^{\alpha}{ }_{\nu}\right)\right]\right\} \\
& =-\exp \left\{\operatorname{Tr}\left[\kappa h^{\alpha}{ }_{\nu}-\frac{\kappa^{2}}{2} h^{\alpha}{ }_{\beta} h^{\beta}{ }_{\nu}\right]\right\} \\
& =-\exp \left\{\kappa h^{\alpha}{ }_{\alpha}-\frac{\kappa^{2}}{2} h^{\alpha}{ }_{\beta} h^{\beta}{ }_{\alpha}\right\} \\
& =-1-\kappa h^{\alpha}{ }_{\alpha}+\frac{\kappa^{2}}{2}\left(h^{\alpha}{ }_{\beta} h^{\beta}{ }_{\alpha}-\frac{1}{2} h_{\alpha}^{\alpha} h_{\beta}{ }_{\beta}\right)
\end{aligned}
$$

em que,

$$
\sqrt{-g} \cong 1+\frac{\kappa}{2} h_{\alpha}^{\alpha}+\frac{\kappa^{2}}{4}\left(\frac{1}{2} h_{\alpha}^{\alpha} h_{\beta}^{\beta}-h_{\beta}^{\alpha} h_{\alpha}^{\beta}\right)+\cdots
$$

Assim, temos usando (3.39) e (3.41) e escrevendo como foi feito na seção anterior, que o produto $\sqrt{-g} g^{\mu \nu}$ será:

$$
\sqrt{-g} g^{\mu \nu} \equiv \eta^{\mu \nu}-h^{\mu \nu}+\frac{1}{2} \eta^{\mu \nu} h_{\alpha}^{\alpha}+\cdots
$$


Neste momento, estamos absorvendo $\kappa$ em $h_{\mu \nu}$ por uma questão de simplicidade (neste caso, $h_{\mu \nu}$ passa a ser admensional). Ao fazermos isso, temos que a ação (3.37), pode ser escrita em termos da expansão (3.42), da seguinte forma,

$$
S=S^{(0)}+S^{(1)}+S^{(2)}+\cdots+S^{(n)},
$$

em que, os índices de cada termo expressam sua ordem em $h$. Desse modo obtemos,

$$
\begin{aligned}
S^{(0)} & =\frac{1}{2} \int \mathrm{d}^{4} x \eta^{\mu \nu} \partial_{\mu} \phi \partial_{\nu} \phi, \\
S^{(1)} & =\frac{1}{2} \int \mathrm{d}^{4} x\left(\frac{1}{2} \eta^{\mu \nu} h_{\alpha}^{\alpha}-h^{\mu \nu}\right) \partial_{\mu} \phi \partial_{\nu} \phi .
\end{aligned}
$$

Os termos subseqüentes $\left(S^{(2)}, S^{(3)}, \cdots, S^{(n)}\right)$ produzem uma série de ações, as quais descrevem a interação de dois campos escalares e qualquer número de grávitons.

\subsubsection{Método alternativo}

Vamos agora mostrar como as contribuições $S^{(1)}, S^{(2)}, S^{(3)}, \cdots$, podem ser obtidas usando a invariância da ação sob transformações de coordenadas. Isso pode ser feito de forma análoga a que foi feita na seção anterior, quando afirmamos que a invariância da ação faz com que o termo de ordem $h^{n}$ esteja relacionado ao de ordem $h^{n+1}$. Supondo que não conhecemos a ação que descreve a interação entre grávitons e campos escalares, teríamos a seguinte forma para a ação $S^{(n)}$,

$$
\begin{aligned}
S^{(0)} & =\frac{1}{2} \int \mathrm{d}^{4} x \eta^{\mu \nu} \partial_{\mu} \phi \partial_{\nu} \phi \\
S^{(1)} & =\frac{1}{2} \int \mathrm{d}^{4} x C^{(1) \mu \nu \mu_{1} \nu_{1}} h_{\mu_{1} \nu_{1}} \partial_{\mu} \phi \partial_{\nu} \phi \\
S^{(2)} & =\frac{1}{2} \int \mathrm{d}^{4} x C^{(2) \mu \nu \mu_{1} \nu_{1} \mu_{2} \nu_{2}} h_{\mu_{1} \nu_{1}} h_{\mu_{2} \nu_{2}} \partial_{\mu} \phi \partial_{\nu} \phi \\
\vdots & \\
S^{(n)} & =\frac{1}{2} \int \mathrm{d}^{4} x C^{(n) \mu \nu \mu_{1} \nu_{1} \cdots \mu_{n} \mu_{n}} h_{\mu_{1} \nu_{1}} \cdots h_{\mu_{n} \nu_{n}} \partial_{\mu} \phi \partial_{\nu} \phi .
\end{aligned}
$$

Neste ponto, estamos apenas supondo que as estruturas $C^{(n) \mu \nu \mu_{1} \nu_{1} \cdots \mu_{n} \mu_{n}}$ são tensores de Lorentz. Deste modo, cada forma $S^{(n)}$ é automaticamente um escalar de Lorentz. 
Para pensarmos numa forma mais geral de escrevermos os tensores $C^{(n)}$, como por exemplo, $C^{(1)}$ e $C^{(2)}$, precisamos analisar os termos em (3.45). Esta análise nos leva a concluir que tais tensores devem ser considerados simétricos na troca $\mu \leftrightarrow \nu$ e $\mu_{n} \leftrightarrow \nu_{n}$ com $n=1,2,3, \cdots$, uma vez que o produto das derivadas do campo escalar e o tensor $h_{\mu_{n} \nu_{n}}$ são simétricos na troca de seus índices. Levando em conta a dimensionalidade da ação, em unidades naturais, podemos chegar a conclusão de que o único objeto que pode ser usado para manter cada expressão em (3.45) invariante de Lorentz, é a métrica de Minkowisk $\eta_{\mu \nu}$. Tomando como exemplo $C^{(1)}$ e $C^{(2)}$, temos, que suas bases tensoriais podem ser escritas como uma combinação de produtos da métrica de Minkowski, como mostram as expressões a seguir,

$$
\begin{aligned}
C^{(1) \mu \nu \mu_{1} \nu_{1}}= & a \eta^{\mu \nu} \eta^{\mu_{1} \nu_{1}}+b \eta^{\mu \mu_{1}} \eta^{\nu \nu_{1}}+c \eta^{\mu \nu_{1}} \eta^{\mu_{1} \nu} \\
C^{(2) \mu \nu \mu_{1} \nu_{1} \mu_{2} \nu_{2}}= & c_{1} \eta^{\mu \nu} \eta^{\mu_{1} \nu_{1}} \eta^{\mu_{1} \nu_{1}}+c_{2} \eta^{\mu \nu} \eta^{\mu_{1} \mu_{2}} \eta^{\nu_{1} \nu_{2}}+c_{3} \eta^{\mu \nu_{1}} \eta^{\mu_{1} \nu} \eta^{\mu_{2} \nu_{2}} \\
& +c_{4} \eta^{\mu \nu_{1}} \eta^{\mu_{1} \mu_{2}} \eta^{\nu \nu_{2}}+c_{5} \eta^{\mu \nu_{2}} \eta^{\mu_{1} \nu_{1}} \eta^{\mu_{2} \nu}+c_{6} \eta^{\mu \nu_{2}} \eta^{\mu_{1} \mu_{2}} \eta^{\nu \nu_{1}}
\end{aligned}
$$

De forma análoga, este procedimento pode ser usado para encontrarmos a forma de todos os $C^{(n) 11}$.

Desta forma, para reescrevermos a ação (3.37) em termos de $C^{(n)}$, devemos levar em conta as questões de simetria discutidas acima. Através delas percebemos que para (3.46a) os segundo e terceiro termos devem ser combinados com o mesmo coeficiente, o que nos leva a concluir que $b=c$. De modo que, a expressão para $C^{(1)}$ será

$$
C^{(1) \mu \nu \mu_{1} \nu_{1}}=a \eta^{\mu \nu} \eta^{\mu_{1} \nu_{1}}+b\left(\eta^{\mu \mu_{1}} \eta^{\nu \nu_{1}}+\eta^{\mu \nu_{1}} \eta^{\mu_{1} \nu}\right)
$$

em que, $a$ e $b$ são números a serem determinados.

Ao contrário do que acontece na invariância de cada termo da ação (3.45) sob transformações de Lorentz, as ações individuais $S^{(n)}$ não são invariantes sob transformações de coordenadas gerais (3.11). No entanto, essas transformações implicam em,

$$
\begin{aligned}
\delta h_{\mu \nu} & =\partial_{\mu} w_{\nu}+\partial_{\nu} w_{\mu}+h_{\alpha \nu} \partial_{\mu} w^{\alpha}+w^{\alpha} \partial_{\alpha} h_{\mu \nu}, \\
\delta \phi & =w^{\mu} \partial_{\mu} \phi,
\end{aligned}
$$

\footnotetext{
${ }^{11}$ Este método será muito útil no próximo capítulo, pois ele ilustra o procedimento que será empregado no caso não comutativo.
} 
no qual, $w(x)$ é o mesmo parâmetro infinitesimal introduzido nas transformações de coordenadas, $x^{\mu}=x^{\mu}-w^{\mu}$. Impondo a invariância da ação, sob transformações gerais de coordenadas, usando (3.48) em,

$$
\delta\left[S^{(0)}+S^{(1)}+S^{(2)}+\cdots+S^{(n)}\right]=0 .
$$

obtemos as relações entre $S^{(n)}$ e $S^{(n+1)}$, dadas pela seguinte relação

$$
\left[\delta\left(S^{(n)}+S^{(n+1)}\right)\right]^{(n)}=0 ; \quad n=0,1,2, \cdots,
$$

em que, o expoente externo é um índice que denota a ordem em $h$ da variação $\delta$. Podemos então escrever (3.50), para cada ordem da variação como segue,

$$
\begin{gathered}
\left\{\delta\left[S^{(0)}+S^{(1)}\right]\right\}^{(0)}=0 \\
\left\{\delta\left[S^{(1)}+S^{(2)}\right]\right\}^{(1)}=0, \\
\vdots \\
\left\{\delta\left[S^{(n)}+S^{(n+1)}\right]\right\}^{(n)}=0 .
\end{gathered}
$$

Estas equações produzem as identidades de Ward a nível de árvore relacionando os vértices $n$ e $n+1$ do gráviton e doi escalares.

Neste momento, somos capazes de determinar as constantes $a$ e $b$ em (3.47). Substituindo (3.45) em (3.51), e tomando a variação de ordem zero em $\delta$, teremos

$$
\delta\left[S^{(0)}+S^{(1)}\right]^{(0)}=\delta\left\{\frac{1}{2} \int \mathrm{d}^{4} x\left[\eta^{\mu \nu}+C^{(1) \mu \nu \mu_{1} \nu_{1}} h_{\mu_{1} \nu_{1}}\right] \partial_{\mu} \phi \partial_{\nu} \phi\right\}
$$




$$
\begin{aligned}
= & \frac{1}{2} \int \mathrm{d}^{4} x\left[\eta^{\mu \nu} \delta\left(\partial_{\mu} \phi \partial_{\nu} \phi\right)+C^{(1) \mu \nu \mu_{1} \nu_{1}} \delta\left(h_{\mu_{1} \nu_{1}} \partial_{\mu} \phi \partial_{\nu} \phi\right)\right] \\
= & \frac{1}{2} \int \mathrm{d}^{4} x\left\{\eta^{\mu \nu}\left[\delta\left(\partial_{\mu} \phi\right) \partial_{\nu} \phi+\partial_{\mu} \phi \delta\left(\partial_{\nu} \phi\right)\right]\right. \\
& \left.+C^{(1) \mu \nu \mu_{1} \nu_{1}}\left[\delta\left(h_{\mu_{1} \nu_{1}}\right) \partial_{\mu} \phi \partial_{\nu} \phi+h_{\mu_{1} \nu_{1}} \delta\left(\partial_{\mu} \phi\right) \partial_{\nu} \phi+h_{\mu_{1} \nu_{1}} \partial_{\mu} \phi \delta\left(\partial_{\nu} \phi\right)\right]\right\} \\
= & \frac{1}{2} \int \mathrm{d}^{4} x\left\{\eta^{\mu \nu}\left[\partial_{\mu}(\delta \phi) \partial_{\nu} \phi+\partial_{\mu} \phi \partial_{\nu}(\delta \phi)\right]\right. \\
& \left.+C^{(1) \mu \nu \mu_{1} \nu_{1}}\left[\delta\left(h_{\mu_{1} \nu_{1}}\right) \partial_{\mu} \phi \partial_{\nu} \phi+h_{\mu_{1} \nu_{1}} \partial_{\mu}(\delta \phi) \partial_{\nu} \phi+h_{\mu_{1} \nu_{1}} \partial_{\mu} \phi \partial_{\nu}(\delta \phi)\right]\right\}
\end{aligned}
$$

em que, as transformações dos campos (3.48) até ordem zero em $h$ fornecem

$$
\begin{aligned}
\delta\left[S^{(0)}+S^{(1)}\right]^{(0)}= & \frac{1}{2} \int \mathrm{d}^{4} x\left\{\eta^{\mu \nu}\left[\partial_{\mu}\left(w^{\alpha} \partial_{\alpha} \phi\right) \partial_{\nu} \phi+\partial_{\mu} \phi \partial_{\nu}\left(w^{\beta} \partial_{\beta} \phi\right)\right]\right. \\
& \left.+C^{(1) \mu \nu \mu_{1} \nu_{1}}\left[\left(\partial_{\mu_{1}} w_{\nu_{1}}+\partial_{\nu_{1}} w_{\mu_{1}}\right) \partial_{\mu} \phi \partial_{\nu} \phi\right]\right\} .
\end{aligned}
$$

Realizando algumas integrações por partes e levando em conta que,

$$
w^{\alpha} \partial_{\alpha} \phi=w^{\nu_{1}} \partial_{\nu_{1}} \phi=\eta^{\mu_{1} \nu_{1}} w_{\mu_{1}} \partial_{\nu_{1}} \phi
$$

temos para (3.52a) a seguinte forma,

$$
\delta\left[S^{(0)}+S^{(1)}\right]^{(0)}=\frac{1}{2} \int \mathrm{d}^{4} x w_{\mu_{1}} \partial_{\nu} \phi \partial_{\nu_{1}}\left(\partial_{\mu} \phi\right)\left[\eta^{\nu_{1} \mu} \eta^{\mu_{1} \nu}+2 C^{(1) \mu \nu \mu_{1} \nu_{1}}\right] .
$$

Neste momento, é conveniente escrever a transformada de Fourier, para $w_{\mu_{1}}$ e para o campo $\phi$,

$$
\begin{aligned}
w_{\mu_{1}}(x) & =\int \mathrm{d}^{4} k \tilde{w}_{\mu_{1}}(k) e^{-i k x} \\
\phi(x) & =\int \mathrm{d}^{4} k \tilde{\phi}(k) e^{-i k x}
\end{aligned}
$$

de forma que a equação (3.52d) será tomada no espaço dos momentos, facilitando nossos cálculos para a obtenção das constantes $a$ e $b$. Assim, (3.52d) no espaço dos momentos 
será,

$$
\begin{aligned}
\delta\left[S^{(0)}+S^{(1)}\right]^{(0)}= & -i \int \mathrm{d}^{4} x \int \mathrm{d}^{4} k_{1} \mathrm{~d}^{4} k_{2} \mathrm{~d}^{4} k_{3} \tilde{w}_{\mu_{1}}\left(k_{1}\right) \tilde{\phi}\left(k_{2}\right) \tilde{\phi}\left(k_{2}\right) e^{-i\left(k_{1}+k_{2}+k_{3}\right) x} \\
& \left\{k_{2 \nu} k_{3 \mu} k_{3 \nu_{1}}\left[\eta^{\nu_{1} \mu} \eta^{\mu_{1} \nu}+2 C^{(1) \mu \nu \mu_{1} \nu_{1}}\right]\right\} \\
= & -i \int \mathrm{d}^{4} k_{1} \mathrm{~d}^{4} k_{2} \mathrm{~d}^{4} k_{3} \tilde{w}_{\mu_{1}}\left(k_{1}\right) \tilde{\phi}\left(k_{2}\right) \tilde{\phi}\left(k_{2}\right) \delta\left(k_{1}+k_{2}+k_{3}\right) \\
& \left\{k_{2 \nu} k_{3 \mu} k_{3 \nu_{1}}\left[\eta^{\nu_{1} \mu} \eta^{\mu_{1} \nu}+2 C^{(1) \mu \nu \mu_{1} \nu_{1}}\right]\right\}
\end{aligned}
$$

em que, os termos $k_{2 \nu}, k_{3 \mu}$ e $k_{3 \nu_{1}}$ carregam os índices referentes as derivadas da exponencial em (3.52d). Usando a equação (3.47), teremos,

$$
\begin{aligned}
k_{3}^{2} k_{2}^{\mu_{1}}+2 a k_{2} \cdot k_{3} k_{3}^{\mu_{1}}+2 b k_{2} \cdot k_{3} k_{3}^{\mu_{1}}+2 b k_{3}^{2} k_{2}^{\mu_{1}} & = \\
k_{3}^{2} k_{2}^{\mu_{1}}(1+2 b)+k_{2} \cdot k_{3} k_{3}^{\mu_{1}}(2 a+2 b) & =0
\end{aligned}
$$

Notando que as estruturas $k_{3}^{2} k_{2}^{\mu_{1}}$ e $k_{2} \cdot k_{3} k_{3}^{\mu_{1}}$ são independentes, obtém-se $b=-\frac{1}{2}$ e $a=-b$, que após serem substituídas em (3.45), fornecem o resultado esperado para a ação dada por,

$$
\begin{aligned}
S & \approx \frac{1}{2} \int \mathrm{d}^{4} x\left\{\eta^{\mu \nu}+\left[\frac{1}{2} \eta^{\mu \nu} \eta^{\mu_{1} \nu_{1}}-\frac{1}{2}\left(\eta^{\mu \mu_{1}} \eta^{\nu \nu_{1}}+\eta^{\mu \nu_{1}} \eta^{\mu_{1} \nu}\right)\right] h_{\mu_{1} \nu_{1}}\right\} \partial_{\mu} \phi \partial_{\nu} \phi \\
& \approx \frac{1}{2} \int \mathrm{d}^{4} x\left\{\eta^{\mu \nu}+\frac{1}{2} \eta^{\mu \nu} \eta^{\mu_{1} \nu_{1}} h_{\mu_{1} \nu_{1}}-\frac{1}{2} \eta^{\mu \mu_{1}} \eta^{\nu \nu_{1}} h_{\mu_{1} \nu_{1}}-\frac{1}{2} \eta^{\mu \nu_{1}} \eta^{\mu_{1} \nu} h_{\mu_{1} \nu_{1}}\right\} \partial_{\mu} \phi \partial_{\nu} \phi \\
& \approx \frac{1}{2} \int \mathrm{d}^{4} x\left\{\eta^{\mu \nu}+\frac{1}{2} \eta^{\mu \nu} h_{\alpha}^{\alpha}-\frac{1}{2} h^{\mu \nu}-\frac{1}{2} h^{\nu \mu}\right\} \partial_{\mu} \phi \partial_{\nu} \phi \\
& \approx \frac{1}{2} \int \mathrm{d}{ }^{4} x\left\{\eta^{\mu \nu}+\frac{1}{2} \eta^{\mu \nu} h_{\alpha}^{\alpha}-h^{\mu \nu}\right\} \partial_{\mu} \phi \partial_{\nu} \phi
\end{aligned}
$$

Comparando este resultado, encontrado pela imposição da invariância por transformações gerais de coordenadas, com o resultado encontrado pela expansão até primeira ordem em $h$, percebemos que nosso método é compatível para reescrevermos a ação (3.37), pelo menos até primeira ordem.

No entanto, para a confirmação de nosso método, precisamos resolver a ordem seguinte, $C^{(2) \mu \nu \mu_{1} \nu_{1} \mu_{2} \nu_{2}}$ usando a simetria na troca de $\mu \rightarrow \nu$ e $\mu_{n} \rightarrow \nu_{n}$. Neste caso, precisaremos 
da variação até primeira ordem em $h$, ou seja, realizaremos o mesmo procedimento para a determinação de (3.56) na equação (3.51b). Com isso temos a seguinte forma para $C^{(2) \mu \nu \mu_{1} \nu_{1} \mu_{2} \nu_{2}} 12$

$$
\begin{aligned}
C^{(2) \mu \nu \mu_{1} \nu_{1} \mu_{2} \nu_{2}}= & {\left[c_{1} \eta^{\mu \nu} \eta^{\mu_{1} \nu_{1}} \eta^{\mu_{1} \nu_{1}}+c_{2} \eta^{\mu \nu} \eta^{\mu_{1} \mu_{2}} \eta^{\nu_{1} \nu_{2}}+c_{3} \eta^{\mu \nu_{1}} \eta^{\mu_{1} \nu} \eta^{\mu_{2} \nu_{2}}+c_{4} \eta^{\mu \nu_{1}} \eta^{\mu_{1} \mu_{2}} \eta^{\nu \nu_{2}}\right.} \\
& \left.+c_{5} \eta^{\mu \nu_{2}} \eta^{\mu_{1} \nu_{1}} \eta^{\mu_{2} \nu}+c_{6} \eta^{\mu \nu_{2}} \eta^{\mu_{1} \mu_{2}} \eta^{\nu \nu_{1}}+\mu_{1} \leftrightarrow \nu_{1}\right]+\mu_{2} \leftrightarrow \nu_{2}
\end{aligned}
$$

em que, levando em conta a simetria pela troca de índices, alguns termos serão combinados com o mesmo coeficiente, o que nos permite escrever $c_{3}=c_{5}$ e $c_{4}=c_{6}$, logo,

$$
\begin{aligned}
C^{(2) \mu \nu \mu_{1} \nu_{1} \mu_{2} \nu_{2}}= & {\left[c_{1} \eta^{\mu \nu} \eta^{\mu_{1} \nu_{1}} \eta^{\mu_{1} \nu_{1}}+c_{2} \eta^{\mu \nu} \eta^{\mu_{1} \mu_{2}} \eta^{\nu_{1} \nu_{2}}+c_{3}\left(\eta^{\mu \nu_{1}} \eta^{\mu_{1} \nu} \eta^{\mu_{2} \nu_{2}}+\eta^{\mu \nu_{2}} \eta^{\mu_{1} \nu_{1}} \eta^{\mu_{2} \nu}\right)\right.} \\
& \left.+c_{4}\left(\eta^{\mu \nu_{1}} \eta^{\mu_{1} \mu_{2}} \eta^{\nu \nu_{2}}+\eta^{\mu \nu_{2}} \eta^{\mu_{1} \mu_{2}} \eta^{\nu \nu_{1}}\right)+\mu_{1} \leftrightarrow \nu_{1}\right]+\mu_{2} \leftrightarrow \nu_{2}
\end{aligned}
$$

Prosseguindo de forma análoga a que foi feita para a determinação das constantes de $C^{(1)}$, temos que as constantes em (3.58) são dadas por

$$
c_{1}=-\frac{1}{16}, \quad c_{2}=c_{3}=\frac{1}{8}, \quad c_{4}=-\frac{1}{4} .
$$

que fornecem o resultado análogo a expansão da ação (3.37) até segunda ordem em $h$.

No entanto, este processo para determinação da ação é dispensável no caso da interação entre campos escalares e grávitons na teoria quântica de campos comutativa. Ele ilustra uma possibilidade alternativa para encontrar a generalização não-comutativa das interações entre campos escalares e grávitons, uma vez que o processo de expansão em campo fraco é bem mais complicado na generalização não comutativa, do que na comutativa.

Veremos no próximo capítulo o quanto este processo será útil para a determinação da ação na generalização não comutativa.

\footnotetext{
${ }^{12}$ Este processo para a obtenção de $C^{(2)}$ é realizado através de cálculos algébricos feitos em comutador, como mostra o apêndice B.2.
} 


\section{Capítulo 4}

\section{Interação entre grávitons e campos}

\section{escalares em espaços não}

\section{comutativos}

\subsection{Os Problemas de uma gravitação não comutativa}

O principal problema para a formulação de uma teoria de gravitação em espaços nãocomutativos, é a dificuldade encontrada na implementação de simetrias na teoria, como por exemplo, a invariância por transformação geral de coordenadas. Esta invariância, é explicitamente violada pela álgebra não-comutativa, que satisfaz a relação (2.2), representada pela seguinte equação.

$$
\left[x^{\mu}, x^{\nu}\right]=i \theta^{\mu \nu}, \quad \mu, \nu=0,1,2,3
$$

A possibilidade de que os efeitos de não-comutatividade possam influenciar as interações gravitacionais tem sido um tema bastante investigado recentemente. Fazendo uma busca em http://www.slac.stanford.edu/spires/hep/, usando palavras tais como "gravity" e "noncommutative" termos uma longa lista de referências. Dentre os diversos trabalhos aqueles que mais se aproximam do enfoque desta dissertação são aqueles que 
tratam da não comutatividade na sua forma mais simples, ou seja, substituindo o produto usual dos campos pelo produto Moyal dado pela equação (2.20).

Uma das primeiras tentativas nessa direção foi feita em [8], ${ }^{1}$ onde a não comutatividade foi introduzida na expansão perturbativa da ação de Einstein substituindo o produto entre campos gravitacionais pelo produto Moyal. No entanto, a invariância sob as transformações de coordenadas não é evidente. Como veremos mais adiante, a transformação geral de coordenadas utilizada na equação (21) de [8] não é compatível com o comutador em (4.1).

Dentre as diversas abordagens mais recentes (com graus distintos de sofisticação matemática) há alguns trabalhos que trataram esse problema utilizando deformações da álgebra de difeomorfismos [20, 21]. Isso permitiu obter a forma da ação até segunda ordem em uma expansão no parâmetro $\theta$. Há ainda abordagens baseadas nas simetrias físicas que produziram resultados distintos para os termos de segunda ordem [22, 23].

No presente capítulo utilizaremos uma abordagem semelhante a que tem sido utilizada pelos autores de [22, 23], porém, no contexto mais simples de campos escalares sem massa, interagindo com grávitons. No entanto, ao invés de restringir a uma expansão no parâmetro $\theta$, procuraremos obter a versão não comutativa determinando a ação ordem a ordem em uma expansão em potências do campo do gráviton $h_{\mu \nu}$ de forma análoga a que foi feita no capítulo anterior. Neste sentido, nossa abordagem é parecida com a que foi utilizada em [8], com a diferença que seremos mais cuidadosos em identificar a simetria da ação.

\footnotetext{
${ }^{1}$ A motivação principal era investigar as propriedades de renormalizabilidade da versão não-comutativa da gravitação, tendo sido concluído que a teoria permanece não renormalizável.
} 


\subsection{Transformação restrita de coordenadas e gravidade unimodular}

A transformação de coordenadas mais geral da teoria de campos comutativa usual no espaço-tempo curvo é

$$
x^{\mu} \rightarrow x^{\mu}=x^{\mu}-w^{\mu} .
$$

Na versão não-comutativa da teoria de campos, sabemos que, as coordenadas do espaço-tempo satisfazem a relação de comutação

$$
\left[x^{\mu}, x^{\nu}\right]=x^{\mu} \star x^{\nu}-x^{\nu} \star x^{\mu}
$$

Analisando estas duas equações, está claro que a transformação (4.2) não pode ser consistente com a equação (4.3) se insistirmos que $w^{\mu}=\tilde{w}^{\mu}(x)$, é uma função geral das coordenadas, e que $\theta^{\mu \nu}$ e independente das coordenadas.

Portanto, podemos explorar as possibilidades de uma simetria local sob transformações infinitesimais com um parâmetro $\tilde{w}^{\mu}(x)$. Para isso, precisamos encontrar uma classe de transformações de coordenadas restrita que preserve a forma de (4.3). Podemos então pensar em uma transformação de coordenadas com parâmetro infinitesimal, $\tilde{w}^{\mu}(x)$, dada por,

$$
x^{\mu}=x^{\mu}-\tilde{w}^{\mu}(x) .
$$

Impondo que a relação de comutação mantenha-se inalterada, ou seja,

$$
\begin{aligned}
{\left[x^{\prime \mu}, x^{\prime \nu}\right]=} & {\left[x^{\mu}-w^{\mu}, x^{\nu}-w^{\nu}\right] } \\
= & x^{\mu} \star x^{\nu}-x^{\mu} \star \tilde{w}^{\nu}-\tilde{w}^{\mu} \star x^{\nu}+ \\
& -x^{\nu} \star x^{\mu}+x^{\nu} \star \tilde{w}^{\mu}+\tilde{w}^{\nu} \star x^{\mu}+\mathcal{O}\left(\tilde{w}^{2}\right) \\
= & {\left[x^{\mu}, x^{\nu}\right]-\left\{\left[x^{\mu}, \tilde{w}^{\nu}\right]+\left[\tilde{w}^{\mu}, x^{\nu}\right]\right\}+\mathcal{O}\left(\tilde{w}^{2}\right) } \\
= & i \theta^{\mu \nu}-\left\{\left[x^{\mu}, \tilde{w}^{\nu}\right]+\left[\tilde{w}^{\mu}, x^{\nu}\right]\right\}+\mathcal{O}\left(\tilde{w}^{2}\right),
\end{aligned}
$$

devemos ter

$$
\left[x^{\mu}, \tilde{w}^{\nu}\right]+\left[\tilde{w}^{\mu}, x^{\nu}\right]=0
$$


ou escrevendo explicitamente,

$$
\left[x^{\mu}, \tilde{w}^{\nu}\right]+\left[\tilde{w}^{\mu}, x^{\nu}\right]=x^{\mu} \star \tilde{w}^{\nu}-\tilde{w}^{\nu} \star x^{\mu}+\tilde{w}^{\mu} \star x^{\nu}-x^{\nu} \star \tilde{w}^{\mu}
$$

e usando o produto Moyal, dado em (2.20), temos,

$$
\begin{aligned}
{\left[x^{\mu}, \tilde{w}^{\nu}\right]+\left[\tilde{w}^{\mu}, x^{\nu}\right]=} & x^{\mu} \tilde{w}^{\nu}+\frac{i}{2} \partial_{\alpha} x^{\mu} \theta^{\alpha \beta} \partial_{\beta} \tilde{w}^{\nu}-\tilde{w}^{\nu} x^{\mu}-\frac{i}{2} \partial_{\alpha} \tilde{w}^{\nu} \theta^{\alpha \beta} \partial_{\beta} x^{\mu} \\
& +\tilde{w}^{\mu} x^{\nu}+\frac{i}{2} \partial_{\alpha} \tilde{w}^{\mu} \theta^{\alpha \beta} \partial_{\beta} x^{\nu}-x^{\nu} \tilde{w}^{\mu}-\frac{i}{2} \partial_{\alpha} x^{\nu} \theta^{\alpha \beta} \partial_{\beta} \tilde{w}^{\mu}
\end{aligned}
$$

Como o produto usual é comutativo, a expressão (4.7b) será,

$$
\begin{aligned}
{\left[x^{\mu}, \tilde{w}^{\nu}\right]+\left[\tilde{w}^{\mu}, x^{\nu}\right]=} & \frac{i}{2} \partial_{\alpha} x^{\mu} \theta^{\alpha \beta} \partial_{\beta} \tilde{w}^{\nu}-\frac{i}{2} \partial_{\alpha} \tilde{w}^{\nu} \theta^{\alpha \beta} \partial_{\beta} x^{\mu} \\
& +\frac{i}{2} \partial_{\alpha} \tilde{w}^{\mu} \theta^{\alpha \beta} \partial_{\beta} x^{\nu}-\frac{i}{2} \partial_{\alpha} x^{\nu} \theta^{\alpha \beta} \partial_{\beta} \tilde{w}^{\mu} \\
= & \frac{i}{2} \delta^{\mu}{ }_{\alpha} \theta^{\alpha \beta} \partial_{\beta} \tilde{w}^{\nu}-\frac{i}{2} \partial_{\alpha} \tilde{w}^{\nu} \theta^{\alpha \beta} \delta^{\mu}{ }_{\beta} \\
& +\frac{i}{2} \partial_{\alpha} \tilde{w}^{\mu} \theta^{\alpha \beta} \delta^{\nu}-\frac{i}{2} \delta^{\nu}{ }_{\alpha} \theta^{\alpha \beta} \partial_{\beta} \tilde{w}^{\mu} \\
= & \frac{i}{2} \theta^{\mu \beta} \partial_{\beta} \tilde{w}^{\nu}-\frac{i}{2} \partial_{\alpha} \tilde{w}^{\nu} \theta^{\alpha \mu}+\frac{i}{2} \partial_{\alpha} \tilde{w}^{\mu} \theta^{\alpha \nu}-\frac{i}{2} \theta^{\nu \beta} \partial_{\beta} \tilde{w}^{\mu} \\
= & i \theta^{\mu \beta} \partial_{\beta} \tilde{w}^{\nu}-i \theta^{\nu \alpha} \partial_{\alpha} \tilde{w}^{\mu} .
\end{aligned}
$$

Sabendo por (4.6) que este resultado é nulo, temos a seguinte equação diferencial,

$$
\theta^{\mu \beta} \partial_{\beta} \tilde{w}^{\nu}=\theta^{\nu \alpha} \partial_{\alpha} \tilde{w}^{\mu}
$$

que deve possuir uma solução que não seja trivial. Desta forma, devemos ter um valor para $\tilde{w}^{\mu}$ diferente de zero. Para isso supomos então que $\tilde{w}^{\mu}$ tenha a seguinte forma,

$$
\tilde{w}^{\mu}(x)=-i\left[x^{\mu}, \Lambda(x)\right]
$$

em que, $\Lambda(x)$ é um parâmetro arbitrário dependente das coordenadas. Substituindo (4.10) em (4.6), temos

$$
\begin{aligned}
{\left[x^{\mu},-i\left[x^{\nu}, \Lambda\right]\right]+\left[-i\left[x^{\mu}, \Lambda\right], x^{\nu}\right] } & =0 \\
-i\left\{\left[x^{\mu},\left[x^{\nu}, \Lambda\right]\right]+\left[\left[x^{\mu}, \Lambda\right], x^{\nu}\right]\right\} & =0 \\
{\left[x^{\mu},\left[\Lambda, x^{\nu}\right]\right]+\left[x^{\nu},\left[x^{\mu}, \Lambda\right]\right] } & =0
\end{aligned}
$$


em que, podemos somar um terceiro termo que possui valor nulo, devido ao fato do comutador interno possuir um valor constante, por (4.1), ou seja,

$$
\left[\Lambda,\left[x^{\nu}, x^{\mu}\right]\right]=-i\left[\Lambda, \theta^{\mu \nu}\right]=0
$$

de modo que escrevemos (4.11) como segue,

$$
\left[x^{\mu},\left[\Lambda, x^{\nu}\right]\right]+\left[x^{\nu},\left[x^{\mu}, \Lambda\right]\right]+\left[\Lambda,\left[x^{\nu}, x^{\mu}\right]\right]=0
$$

que é a identidade de Jacobi.

Com este resultado mostramos que (4.6) é satisfeita por esta tentativa do parâmetro $\tilde{w}^{\mu}(x)$, no entanto, precisamos verificar ainda como podemos escrevê-lo, após desenvolvermos o comutador em (4.10). Desta forma, temos,

$$
\begin{aligned}
\tilde{w}^{\mu}(x) & =-i\left[x^{\mu}, \Lambda(x)\right] \\
& =-i\left(x^{\mu} \star \Lambda(x)-\Lambda(x) \star x^{\mu}\right) \\
& =-i\left[x^{\mu}\left(1+\frac{i}{2} \overleftarrow{\partial}_{\alpha} \theta^{\alpha \beta} \vec{\partial}_{\beta}\right) \Lambda(x)-\Lambda(x)\left(1+\frac{i}{2} \overleftarrow{\partial}_{\alpha} \theta^{\alpha \beta} \vec{\partial}_{\beta}\right) x^{\mu}\right] \\
& =-i\left[x^{\mu} \Lambda(x)+\frac{i}{2} \partial_{\alpha} x^{\mu} \theta^{\alpha \beta} \partial_{\beta} \Lambda(x)-\Lambda(x) x^{\mu}-\frac{i}{2} \partial_{\alpha} \Lambda(x) \theta^{\alpha \beta} \partial_{\beta} x^{\mu}\right] \\
& =-i\left[\frac{i}{2} \delta^{\mu}{ }_{\alpha} \theta^{\alpha \beta} \partial_{\beta} \Lambda(x)-\frac{i}{2} \partial_{\alpha} \Lambda(x) \theta^{\alpha \beta} \delta^{\mu}{ }_{\beta}\right] \\
& =-i\left[\frac{i}{2} \theta^{\mu \beta} \partial_{\beta} \Lambda(x)-\frac{i}{2} \theta^{\alpha \mu} \partial_{\alpha} \Lambda(x)\right] \\
& =\theta^{\mu \beta} \partial_{\beta} \Lambda(x)
\end{aligned}
$$

com $\tilde{w}^{\mu}(x)$ satisfazendo a seguinte condição,

$$
\partial_{\mu} \tilde{w}^{\mu}(x)=\partial_{\mu} \theta^{\mu \beta} \partial_{\beta} \Lambda(x)=\theta^{\mu \beta} \partial_{\mu} \partial_{\beta} \Lambda(x)=0,
$$

em que, temos o produto de um termo anti-simétrico por outro simétrico resultando em um valor nulo, o que já era esperado para que (4.9) tivesse uma solução não trivial.

Desta forma,

$$
\left[x^{\prime \mu}, x^{\prime \nu}\right]=\left[x^{\mu}, x^{\nu}\right]=i \theta^{\mu \nu}
$$

é invariante pelas transformações restritas de coordenadas. 
Esta transformação restrita nos conduzem a importantes consequências, como por exemplo, a determinação do Jacobiano, dada por,

$$
\begin{aligned}
\operatorname{det}\left(\frac{\partial x^{\prime \mu}}{\partial x^{\nu}}\right) & =\operatorname{det}\left(\frac{\partial x^{\mu}}{\partial x^{\nu}}+\frac{\partial \tilde{w}^{\mu}}{\partial x^{\nu}}\right) \\
& =\operatorname{det}\left(\delta_{\nu}^{\mu}+\partial_{\nu} \tilde{w}^{\mu}\right) \\
& =\exp \left[\operatorname{Tr} \ln \left(\delta_{\nu}^{\mu}+\partial_{\nu} \tilde{w}^{\mu}\right)\right] \\
& =\exp \left[\operatorname{Tr}\left(\partial_{\nu} \tilde{w}^{\mu}\right)\right] \\
& =\exp \left(\partial_{\mu} \tilde{w}^{\mu}\right) \\
& =1+\partial_{\mu} \tilde{w}^{\mu}
\end{aligned}
$$

em que,

$$
\operatorname{det}\left(\frac{\partial x^{\prime \mu}}{\partial x^{\nu}}\right)=1
$$

Desta forma, temos com o Jacobiano da transformação igual a um $(J=1)$, que o elemento de volume é invariante, $\mathrm{d}^{4} x^{\prime}=\mathrm{d}^{4} x$, de modo que a teoria baseada na preservação do volume é uma versão da Relatividade Geral conhecida como teoria unimodular da gravitação. Um consequência direta da teoria unimodular da gravitação é a forma como se transforma o determinante da métrica.

Sabemos que na teoria comutativa ele se transforma como um escalar exceto pelo valor extra do Jacobiano, devido ao termo da derivada de $w(x)$, proveniente da transformação geral de coordenadas como verificamos podemos verificar em [18]. Na teoria unimodular, temos que esta derivada se anula como vimos em (4.15). No entanto, para estudarmos a não comutatividade na interação precisamos encontrar a forma como se transforma a raiz do determinante da métrica, dada pela seguinte propriedade,

$$
\delta \sqrt{-g}=\frac{1}{2} \sqrt{-g} g^{\mu \nu} \delta g_{\mu \nu}
$$


Para isso, substituímos a expressão (3.24), em (4.19), e obtemos

$$
\begin{aligned}
\delta \sqrt{-g} & =\frac{1}{2} \sqrt{-g} g^{\mu \nu}\left(g_{\alpha \nu} \partial_{\mu} \tilde{w}^{\alpha}+g_{\alpha \mu} \partial_{\nu} \tilde{w}^{\alpha}+\tilde{w}^{\alpha} \partial_{\alpha} g_{\mu \nu}\right) \\
& =\frac{1}{2} \sqrt{-g}\left(g^{\mu \nu} g_{\alpha \nu} \partial_{\mu} \tilde{w}^{\alpha}+g^{\mu \nu} g_{\alpha \mu} \partial_{\nu} \tilde{w}^{\alpha}+g^{\mu \nu} \tilde{w}^{\alpha} \partial_{\alpha} g_{\mu \nu}\right) \\
& =\frac{1}{2} \sqrt{-g}\left(2 \partial_{\alpha} \tilde{w}^{\alpha}+g^{\mu \nu} \tilde{w}^{\alpha} \partial_{\alpha} g_{\mu \nu}\right) \\
& =\sqrt{-g} \partial_{\alpha} \tilde{w}^{\alpha}+\tilde{w}^{\alpha} \frac{1}{2} \sqrt{-g} g^{\mu \nu} \partial_{\alpha} g_{\mu \nu} \\
& =\sqrt{-g} \partial_{\alpha} \tilde{w}^{\alpha}+\tilde{w}^{\alpha} \partial_{\alpha} \sqrt{-g} \\
& =\tilde{w}^{\alpha} \partial_{\alpha} \sqrt{-g} .
\end{aligned}
$$

Com esta transformação restrita de coordenadas verificamos em (4.20) que a raiz do determinante da métrica se transforma como um escalar, de maneira análoga a (3.48b). O que nos leva a escrever a seguinte condição,

$$
\delta \sqrt{-g}=0
$$

uma vez que, $g=-1$ implicando em $\sqrt{-g}=1$ que é um valor que satisfaz a condição de matriz unimodular como verificamos na referência [18]. Assim, a variação da ação fica vinculada pela equação (4.21). Na próxima seção esta condição nos ajudará a interpretar os cálculos usados na determinação da ação não-comutativa.

Em [22] acredita-se que a tentativa de uma unificação da Mecânica Quântica com a Relatividade Geral requer uma pequena modificação na distância do espaço-tempo, ou seja, supõe-se a existência de um comprimento fundamental. No entanto, os modelos em teorias de gauge que incorporam a noção de um comprimento fundamental são os formulados em espaços não comutativos, mas o principal problema na formulação de uma teoria de gravitação em espaços não-comutativos é a implementação de simetrias, como a por transformações gerais de coordenadas.

Como aparentemente resolvemos a dificuldade gerada pela implementação da simetria por transformações de coordenadas, podemos tentar, de uma forma simples mas com consequências profundas, construir uma teoria de interação gravitacional em espaços não comutativos. 
Os resultados obtidos nesta seção são muito importantes para nossa tentativa, uma vez que as simetrias do espaço-tempo não comutativo indicam o caminho para uma versão da interação entre grávitons e campos escalares não comutativos. Neste sentido, as condições impostas pela gravitação unimodular, nos ajudarão a determinar a forma da ação não comutativa que será calculada na próxima seção.

\subsection{Cálculo da ação em espaços não comutativos}

No capítulo anterior analisamos o contexto de uma teoria comutativa com interação entre grávitons e campos escalares. Mostramos ainda na seção anterior que as simetrias por transformações gerais de coordenadas nos conduziram a uma versão não comutativa da gravitação unimodular. Com base nestas descobertas vamos investigar como ocorre a interação entre grávitons e campos escalares na representação de espaços não comutativos.

Vimos que a ação (3.37) pode ser escrita usando-se a invariância por transformações gerais de coordenadas. No entanto, a forma desta ação não foi verificada apenas pela expansão da métrica $g^{\mu \nu}$. Usamos um outro método para obter (3.37), e concluímos que ele foi capaz de reescrever a ação.

Iremos então seguir os passos realizados no capítulo anterior para tentarmos escrever a ação de interação entre grávitons e campos escalares neste novo espaço, ou seja, substituindo o produto usual pelo produto Moyal.

Reescrevendo (3.45) como segue,

$$
\begin{aligned}
S^{(0)} & =\frac{1}{2} \int \mathrm{d}^{4} x \eta^{\mu \nu} \partial_{\mu} \phi \star \partial_{\nu} \phi, \\
S^{(1)} & =\frac{1}{2} \int \mathrm{d}^{4} x C^{(1) \mu \nu \mu_{1} \nu_{1}} h_{\mu_{1} \nu_{1}} \star \partial_{\mu} \phi \star \partial_{\nu} \phi, \\
S^{(2)} & =\frac{1}{2} \int \mathrm{d}^{4} x C^{(2) \mu \nu \mu_{1} \nu_{1} \mu_{2} \nu_{2}} h_{\mu_{1} \nu_{1}} \star h_{\mu_{2} \nu_{2}} \star \partial_{\mu} \phi \star \partial_{\nu} \phi, \\
\vdots & \\
S^{(n)} & =\frac{1}{2} \int \mathrm{d}^{4} x C^{(n) \mu \nu \mu_{1} \nu_{1} \cdots \mu_{n} \mu_{n}} h_{\mu_{1} \nu_{1}} \star \cdots \star \mu_{n} \nu_{n} \star \partial_{\mu} \phi \star \partial_{\nu} \phi,
\end{aligned}
$$


temos a versão não comutativa para o método adotado no capítulo anterior

Tomando as relações (3.51) modificadas pela introdução do produto *

$$
\begin{gathered}
\left\{\delta\left[S^{(0)}+S^{(1)}\right]_{\star}\right\}^{(0)}=0 \\
\left\{\delta\left[S^{(1)}+S^{(2)}\right]_{\star}\right\}^{(1)}=0 \\
\vdots \\
\left\{\delta\left[S^{(n)}+S^{(n+1)}\right]_{\star}\right\}^{(n)}=0
\end{gathered}
$$

podemos encontrar a forma da ação, quando soubermos os valores das constantes $a$ e $b$ substituirmos na equação (3.47), expressa por,

$$
C^{(1) \mu \nu \mu_{1} \nu_{1}}=a \eta^{\mu \nu} \eta^{\mu_{1} \nu_{1}}+b\left(\eta^{\mu \mu_{1}} \eta^{\nu \nu_{1}}+\eta^{\mu \nu_{1}} \eta^{\mu_{1} \nu}\right)
$$

em (4.23), já que este foi o procedimento adotado para a investigação da ação comutativa. Seguindo tal procedimento, substituímos em (4.23a) as expressões equivalentes de (4.22), para obtermos,

$$
\begin{aligned}
\delta\left[S^{(0)}+S^{(1)}\right]_{\star}{ }^{(0)}= & \frac{1}{2} \int \mathrm{d}^{4} x \delta\left\{\eta^{\mu \nu} \partial_{\mu} \phi \star \partial_{\nu} \phi+C^{(1) \mu \nu \mu_{1} \nu_{1}} h_{\mu_{1} \nu_{1}} \star \partial_{\mu} \phi \star \partial_{\nu} \phi\right\}, \\
= & \frac{1}{2} \int \mathrm{d}^{4} x\left\{\eta^{\mu \nu}\left[\delta\left(\partial_{\mu} \phi\right) \star \partial_{\nu} \phi+\partial_{\mu} \phi \star \delta\left(\partial_{\nu} \phi\right)\right]\right. \\
& \left.+C^{(1) \mu \nu \mu_{1} \nu_{1}} \delta\left(h_{\mu_{1} \nu_{1}} \star \partial_{\mu} \phi \star \partial_{\nu} \phi\right)\right\} \\
= & \frac{1}{2} \int \mathrm{d}^{4} x\left\{\eta^{\mu \nu}\left[\partial_{\mu}(\delta \phi) \star \partial_{\nu} \phi+\partial_{\mu} \phi \star \partial_{\nu}(\delta \phi)\right]\right. \\
& \left.+C^{(1) \mu \nu \mu_{1} \nu_{1}} \delta h_{\mu_{1} \nu_{1}} \star \partial_{\mu} \phi \star \partial_{\nu} \phi\right\}
\end{aligned}
$$


em que, tomando as seguintes transformação para os campos do gráviton e escalares²,

$$
\begin{aligned}
\delta \phi & =\frac{1}{2}\left\{w^{\alpha}, \partial_{\alpha} \phi\right\}, \\
\delta h_{\alpha \beta} & =\partial_{\alpha} \tilde{w}_{\beta}+\partial_{\beta} \tilde{w}_{\alpha},
\end{aligned}
$$

e considerando apenas termos de ordem zero, teremos,

$$
\begin{gathered}
\delta\left[S^{(0)}+S^{(1)]_{\star}{ }^{(0)}=} \frac{1}{2} \int \mathrm{d}^{4} x\left\{\eta^{\mu \nu}\left[\partial_{\mu}\left(\frac{1}{2}\left\{\tilde{w}^{\alpha}, \partial_{\alpha} \phi\right\}\right) \star \partial_{\nu} \phi+\partial_{\mu} \phi \star \partial_{\nu}\left(\frac{1}{2}\left\{\tilde{w}^{\alpha}, \partial_{\alpha} \phi\right\}\right)\right]\right.\right. \\
\left.+C^{(1) \mu \nu \mu_{1} \nu_{1}}\left(\partial_{\mu_{1}} \tilde{w}_{\nu_{1}}+\partial_{\nu_{1}} \tilde{w}_{\mu_{1}}\right) \star \partial_{\mu} \phi \star \partial_{\nu} \phi\right\}, \\
=\frac{1}{2} \int \mathrm{d}^{4} x\left\{\eta^{\mu \nu} \frac{1}{2}\left[\partial_{\mu}\left\{\tilde{w}^{\alpha}, \partial_{\alpha} \phi\right\} \star \partial_{\nu} \phi+\partial_{\mu} \phi \star \partial_{\nu}\left\{\tilde{w}^{\alpha}, \partial_{\alpha} \phi\right\}\right]\right. \\
\left.+C^{(1) \mu \nu \alpha \beta}\left(\partial_{\alpha} \tilde{w}_{\beta} \star \partial_{\mu} \phi \star \partial_{\nu} \phi+\partial_{\beta} \tilde{w}_{\alpha} \star \partial_{\mu} \phi \star \partial_{\nu} \phi\right)\right\} .
\end{gathered}
$$

Usando a propriedade de ciclicidade do traço, dada em (2.25), e a simetria em $C^{(1) \mu \nu \alpha \beta}$ na troca de $\alpha \leftrightarrow \beta$, segue que,

$$
\begin{aligned}
& \delta\left[S^{(0)}+S^{(1)}\right]_{\star}{ }^{(0)}=\frac{1}{2} \int \mathrm{d}^{4} x\left\{\eta^{\mu \nu} \partial_{\mu} \phi \star \partial_{\nu}\left\{\tilde{w}^{\alpha}, \partial_{\alpha} \phi\right\}\right. \\
& \left.+2 C^{(1) \mu \nu \alpha \beta} \partial_{\beta} \tilde{w}_{\alpha} \star \partial_{\mu} \phi \star \partial_{\nu} \phi\right\} . \\
& =\frac{1}{2} \int \mathrm{d}^{4} x\left\{\eta^{\mu \nu} \partial_{\mu} \phi \star \partial_{\nu}\left\{\tilde{w}^{\alpha}, \partial_{\alpha} \phi\right\}+2 C^{(1) \mu \nu \alpha \beta} \partial_{\beta} \tilde{w}_{\alpha} \star \partial_{\mu} \phi \star \partial_{\nu} \phi\right\} .
\end{aligned}
$$

é a forma da variação de ordem zero em $\delta$ para o caso não comutativo. Agora usando o

${ }^{2}$ Estamos aqui adotando a prescrição,

$$
w^{\alpha} \partial_{\alpha} \phi \rightarrow \frac{1}{2}\left(w^{\alpha} \star \partial_{\alpha} \phi-\partial_{\alpha} \phi \star w^{\alpha}\right)
$$

que é simétrica sob $\theta_{\mu \nu} \rightarrow-\theta_{\nu \mu}$ 
resultado encontrado em (4.14), teremos a seguinte equação para (4.28b),

$$
\begin{aligned}
& \delta\left[S^{(0)}+S^{(1)}\right]_{\star}{ }^{(0)}= \frac{1}{2} \int \mathrm{d}^{4} x\left\{\eta^{\mu \nu} \partial_{\mu} \phi \star \partial_{\nu}\left\{\theta^{\alpha \beta} \partial_{\beta} \Lambda, \partial_{\alpha} \phi\right\}\right. \\
&\left.+2 C^{(1) \mu \nu \alpha \beta} \partial_{\beta}\left(\theta_{\alpha \lambda} \partial^{\lambda} \Lambda\right) \star \partial_{\mu} \phi \star \partial_{\nu} \phi\right\} \\
&=\frac{1}{2} \int \mathrm{d}^{4} x\left\{\eta^{\mu \nu} \partial_{\mu} \phi \star \partial_{\nu}\left\{\theta^{\alpha \beta} \partial_{\beta} \Lambda, \partial_{\alpha} \phi\right\}\right\} \\
&+\frac{1}{2} \int \mathrm{d}^{4} x\left\{2\left[a \eta^{\mu \nu} \eta^{\alpha \beta}+b\left(\eta^{\mu \alpha} \eta^{\nu \beta}+\eta^{\mu \beta} \eta^{\nu \alpha}\right)\right] \theta_{\alpha \lambda} \partial_{\beta} \partial^{\lambda} \Lambda \star \partial_{\mu} \phi \star \partial_{\nu} \phi\right\}
\end{aligned}
$$

Analisando o resultado em (4.29b) percebemos que o parâmetro $a$ não pode ser determinado pela transformação de coordenadas restrita, uma vez que o termo gerado após a contração dos índices, é formado pelo produto de um termo anti-simétrico com um termo simétrico, ou seja,

$$
a \eta^{\mu \nu} \eta^{\alpha \beta} \theta_{\alpha \lambda} \partial_{\beta} \partial^{\lambda} \Lambda=a \eta^{\mu \nu} \theta^{\alpha \lambda} \partial_{\beta} \partial_{\lambda} \Lambda=0
$$

Desta forma, não é possível determinar a constante $a$ em (4.29b). Prosseguindo, quando contraímos os termos que acompanham a constate $b$ e desenvolvemos o anti-comutador em (4.29b), temos,

$$
\begin{aligned}
\delta\left[S^{(0)}+S^{(1)}\right]_{\star}{ }^{(0)}= & \frac{1}{2} \theta^{\alpha \beta} \int \mathrm{d}^{4} x\left\{\eta^{\mu \nu} \partial_{\mu} \phi \star \partial_{\nu}\left[\partial_{\beta} \Lambda \star \partial_{\alpha} \phi+\partial_{\alpha} \phi \star \partial_{\beta} \Lambda\right]\right\} \\
& +\theta_{\alpha \lambda} \int \mathrm{d}^{4} x\left\{b \left[\eta^{\mu \alpha} \eta^{\nu \beta} \partial_{\beta} \partial^{\lambda} \Lambda \star \partial_{\mu} \phi \star \partial_{\nu} \phi\right.\right. \\
& \left.\left.+\eta^{\mu \beta} \eta^{\nu \alpha} \partial_{\beta} \partial^{\lambda} \Lambda \star \partial_{\mu} \phi \star \partial_{\nu} \phi\right]\right\}
\end{aligned}
$$




$$
\begin{aligned}
\delta\left[S^{(0)}+S^{(1)}\right]_{\star}{ }^{(0)}= & \frac{1}{2} \theta^{\alpha \beta} \int \mathrm{d}^{4} x\left\{\eta^{\mu \nu} \partial_{\mu} \phi \star \partial_{\nu} \partial_{\beta} \Lambda \star \partial_{\alpha} \phi\right. \\
& +\eta^{\mu \nu} \partial_{\mu} \phi \star \partial_{\beta} \Lambda \star \partial_{\nu} \partial_{\alpha} \phi \\
& +\eta^{\mu \nu} \partial_{\mu} \phi \star \partial_{\nu} \partial_{\alpha} \phi \star \partial_{\beta} \Lambda \\
& \left.+\eta^{\mu \nu} \partial_{\mu} \phi \star \partial_{\alpha} \phi \star \partial_{\nu} \partial_{\beta} \Lambda\right\} \\
& +\theta_{\alpha \lambda} \int \mathrm{d}^{4} x\left\{b \left[\partial_{\beta} \partial^{\lambda} \Lambda \star \partial^{\alpha} \phi \star \partial^{\beta} \phi\right.\right. \\
& \left.\left.+\partial_{\beta} \partial^{\lambda} \Lambda \star \partial^{\beta} \phi \star \partial^{\alpha} \phi\right]\right\} .
\end{aligned}
$$

Logo, analisando (4.29e) é possível mostrar pelas propriedades de ciclicidade do traço e por integração por partes que os, segundo e terceiro, termos da primeira integral se cancelam. Desta forma, podemos escrever esta equação como segue,

$$
\begin{aligned}
\delta\left[S^{(0)}+S^{(1)}\right]_{\star}{ }^{(0)}= & \frac{1}{2} \theta^{\alpha \beta} \int \mathrm{d}^{4} x\left\{\eta^{\mu \nu} \partial_{\mu} \phi \star \partial_{\nu} \partial_{\beta} \Lambda \star \partial_{\alpha} \phi\right. \\
& \left.+\eta^{\mu \nu} \partial_{\mu} \phi \star \partial_{\alpha} \phi \star \partial_{\nu} \partial_{\beta} \Lambda\right\} \\
& +b \theta_{\alpha \lambda} \int \mathrm{d}^{4} x\left\{\partial_{\beta} \partial^{\lambda} \Lambda \star \partial^{\alpha} \phi \star \partial^{\beta} \phi\right. \\
& \left.+\partial_{\beta} \partial^{\lambda} \Lambda \star \partial^{\beta} \phi \star \partial^{\alpha} \phi\right\},
\end{aligned}
$$

em que, podemos ajeitá-la de forma que encontremos o valor da constante $b$. Para isso, reescreveremos a segunda integral utilizando a propriedade de ciclicidade do traço e introduzindo a métrica de Minkowski contravariante para organizar os índices.Esta 
equação fica então da seguinte forma,

$$
\begin{aligned}
\delta\left[S^{(0)}+S^{(1)}\right]_{\star}{ }^{(0)}= & \frac{1}{2} \theta^{\alpha \beta} \int \mathrm{d}^{4} x\left\{\eta^{\mu \nu} \partial_{\mu} \phi \star \partial_{\nu} \partial_{\beta} \Lambda \star \partial_{\alpha} \phi\right. \\
& \left.+\eta^{\mu \nu} \partial_{\mu} \phi \star \partial_{\alpha} \phi \star \partial_{\nu} \partial_{\beta} \Lambda\right\} \\
& +b \theta^{\alpha \beta} \int \mathrm{d}^{4} x\left\{\eta^{\mu \nu} \partial_{\mu} \phi \star \partial_{\nu} \partial_{\beta} \Lambda \star \partial_{\alpha} \phi\right. \\
& \left.+\eta^{\mu \nu} \partial_{\mu} \phi \star \partial_{\alpha} \phi \star \partial_{\nu} \partial_{\beta} \Lambda\right\},
\end{aligned}
$$

em que, encontramos o valor de $b=-\frac{1}{2}$, pela imposição de que (4.31) seja nulo.

Neste momento, podemos tomar as equações (3.43) e (4.22), como segue,

$$
\begin{aligned}
S \approx & \frac{1}{2} \int \mathrm{d}^{4} x\left[\eta^{\mu \nu} \partial_{\mu} \phi \star \partial_{\nu} \phi+C^{(1) \mu \nu \mu_{1} \nu_{1}} h_{\mu_{1} \nu_{1}} \star \partial_{\mu} \phi \star \partial_{\nu} \phi\right], \\
S \approx & \frac{1}{2} \int \mathrm{d}^{4} x\left\{\eta^{\mu \nu} \partial_{\mu} \phi \star \partial_{\nu} \phi+\left[a \eta^{\mu \nu} \eta^{\mu_{1} \nu_{1}}\right.\right. \\
& \left.\left.+b\left(\eta^{\mu \mu_{1}} \eta^{\nu \nu_{1}}+\eta^{\mu \nu 1} \eta^{\mu_{1} \nu}\right)\right] h_{\mu_{1} \nu_{1}} \star \partial_{\mu} \phi \star \partial_{\nu} \phi\right\} \\
S \approx & \frac{1}{2} \int \mathrm{d}^{4} x\left\{\eta^{\mu \nu} \partial_{\mu} \phi \star \partial_{\nu} \phi+\left[a \eta^{\mu \nu} \eta^{\mu_{1} \nu_{1}} h_{\mu_{1} \nu_{1}} \star \partial_{\mu} \phi \star \partial_{\nu} \phi\right.\right. \\
& \left.\left.+b\left(\eta^{\mu \mu_{1}} \eta^{\nu \nu_{1}} h_{\mu_{1} \nu_{1}} \star \partial_{\mu} \phi \star \partial_{\nu} \phi+\eta^{\mu \nu_{1}} \eta^{\mu_{1} \nu} h_{\mu_{1} \nu_{1}} \star \partial_{\mu} \phi \star \partial_{\nu} \phi\right)\right]\right\}, \\
S \approx & \frac{1}{2} \int \mathrm{d}^{4} x\left\{\eta^{\mu \nu} \partial_{\mu} \phi \star \partial_{\nu} \phi+\left[a \eta^{\mu \nu} \eta^{\mu_{1} \nu_{1}} h_{\mu_{1} \nu_{1}} \star \partial_{\mu} \phi \star \partial_{\nu} \phi\right.\right. \\
& \left.\left.+b\left(\eta^{\mu \mu_{1}} \eta^{\nu \nu_{1}} h_{\mu_{1} \nu_{1}} \star \partial_{\mu} \phi \star \partial_{\nu} \phi+\eta^{\mu \nu_{1}} \eta^{\mu_{1} \nu} h_{\mu_{1} \nu_{1}} \star \partial_{\mu} \phi \star \partial_{\nu} \phi\right)\right]\right\},
\end{aligned}
$$

e substituindo o valor de $b$, teremos

$$
\begin{aligned}
S \approx & \frac{1}{2} \int \mathrm{d}^{4} x\left\{\eta^{\mu \nu} \partial_{\mu} \phi \star \partial_{\nu} \phi+a \eta^{\mu \nu} h_{\nu_{1}}^{\nu_{1}} \star \partial_{\mu} \phi \star \partial_{\nu} \phi\right. \\
& \left.-\frac{1}{2}\left(h^{\mu \nu} \star \partial_{\mu} \phi \star \partial_{\nu} \phi+h^{\nu \mu} \star \partial_{\mu} \phi \star \partial_{\nu} \phi\right)\right\}
\end{aligned}
$$

O valor de $a$ não foi substituído em (4.32d), devido ao fato de não podermos determinálo, pela condição em (4.29c). No entanto, temos ainda que a condição segundo a qual o limite de $\theta \rightarrow 0$, deve fornecer o resultado dado por (3.37). De acordo com o valor 
obtido no capítulo anterior esse resultado deve ser $a=-b$. Assim, no limite de $\theta \rightarrow 0$, temos que a expressão $(4.32 \mathrm{~d})$ concorda com o caso comutativo para a ação. No entanto, como veremos a seguir, este valor de a não contribuirá para a construção da ação não comutativa, uma vez que o termo que acompanha tal constante possui valor nulo.

Mostraremos que não estamos em contradição com o caso comutativo, pois ao tomarmos a equação (3.41), dada por,

$$
\sqrt{-g} \cong 1+\frac{\kappa}{2} h_{\alpha}^{\alpha}+\frac{\kappa^{2}}{4}\left(\frac{1}{2} h_{\alpha}^{\alpha} h_{\beta}^{\beta}-h_{\beta}^{\alpha} h_{\alpha}^{\beta}\right)+\cdots,
$$

em que, pela condição de unimodularidade, o valor da raiz do determinante da métrica deve ser $\sqrt{-g}=1$, como verificado na seção anterior, o que nos leva a concluir que o traço de $h$ é $h_{\alpha}^{\alpha}=0$, ou seja, o termo de primeira ordem em (4.33) que acompanha a constante $a$ na ação, deve ser nulo. Desta forma, a equação (4.32d) será

$$
\begin{aligned}
S & \approx \frac{1}{2} \int \mathrm{d}^{4} x\left\{\eta^{\mu \nu} \partial_{\mu} \phi \star \partial_{\nu} \phi-\frac{1}{2}\left(h^{\mu \nu} \star \partial_{\mu} \phi \star \partial_{\nu} \phi+h^{\nu \mu} \star \partial_{\mu} \phi \star \partial_{\nu} \phi\right)\right\}, \\
& \approx \frac{1}{2} \int \mathrm{d}^{4} x\left(\eta^{\mu \nu} \partial_{\mu} \phi \star \partial_{\nu} \phi-\frac{1}{2} h^{\mu \nu} \star\left\{\partial_{\mu} \phi, \partial_{\nu} \phi\right\}\right),
\end{aligned}
$$

que é a forma da ação não comutativa até primeira ordem em $h$.

De forma análoga à desenvolvida até aqui, é possível, em princípio, encontrarmos as ordens superiores e verificarmos que no limite de $\theta \rightarrow 0$, concordam com a forma comutativa da ação de interação entre grávitons e campos escalares.

Com base nestes resultados, nosso método mostrou que é possível construirmos uma ação não comutativa para esta interação simples. No entanto, ele nos fornece também interpretações profundas, uma vez que a introdução da unimodularidade é fundamental para termos uma ação que seja invariante por transformações de coordenadas, mas para isso é preciso que ela seja uma transformação restrita, ou seja, temos uma imposição da teoria. 


\section{Capítulo 5}

\section{Considerações finais}

A Ação é a construção matemática que permite o entendimento das leis fundamentais da física, uma vez que produz as equações de movimento, e a análise de sua invariância nos levam a quantidades conservadas.

Neste trabalho, um problema que tentamos entender foi a possibilidade de que os efeitos de não comutatividade possam influenciar também as interações gravitacionais. O primeiro passo para iniciar esta investigação constitui em saber qual seria a forma da ação que descreve as interações entre campos escalar e gravitacional, quando a não comutatividade é levada em conta. Este problema tem sido investigado em diversos trabalhos com diferentes níveis de profundidade. Em vários trabalhos recentes (ver por exemplo $\{[24],[25]\}$ e referências lá citadas), tem sido investigada a possibilidade de se obter a generalização não comutativa da ação de Einstein-Hilbert. Tendo em vista as dificuldades envolvidas, os resultados existentes se restringem a uma expansão em potências dos parâmetros $\theta^{\mu \nu}$. Nossa abordagem é de certa forma complementar, na medida em que tratamos especificamente das interações entre grávitons e os bosons escalares, porém, sem nos restringirmos a uma expansão em $\theta^{\mu \nu}$. A técnica que utilizamos visa obter sistematicamente (utilizando as identidades de Ward associadas à simetria restrita) a expansão de campo gravitacional fraco. 
Ao analisarmos a invariância da ação sob as transformações (3.25) na seção (3.2.1), vimos que a ordem mais baixa da expansão da ação de Einstein-Hilbert, dada por (3.27), é obtida. Mencionamos que este procedimento poderia ser continuado até obtermos as ordens superiores. Assim, aplicamos este procedimento na construção da versão não comutativa da ação (3.37) que é o modelo mais simples envolvendo interações gravitacionais.

Esta construção foi realizada a partir da substituição do produto usual pelo produto Moyal, no método alternativo que desenvolvido na seção (3.3.1), dado pelas equações (3.45). Nestas equações, impomos que cada $S^{(n)}$ era um escalar de Lorentz, o que nos levou a supor estruturas $C^{(n) \mu \nu \mu_{1} \nu_{1} \cdots \mu_{n} \mu_{n}}$ que envolviam combinações do produto da métrica de Minkowisk, ou seja, tínhamos construído tensores de Lorentz para mantermos a invariância de casa $S^{(n)}$ por transformações de Lorentz.

Com a substituição do produto usual pelo produto Moyal, as equações (3.45) foram reescritas de modo que, sua forma é dada por (4.22), na qual a ordem zero é simplesmente a teoria escalar livre, uma vez que o produto $\star$ não modifica a parte quadrática como vimos em (2.25). Note, no entanto, que as ações individuais $S^{(n)}$ não são invariantes de Lorentz no caso não comutativo. Mesmo assim, é possível ter, em princípio, uma simetria local que relaciona $S^{(n)}$ com $S^{(n+1)}$. Isso ocorre, por exemplo, em teorias de campos de gauge a temperatura finita. De fato, a ação efetiva térmica dessas teorias possui invariância de gauge local, mas a simetria de Lorentz é quebrada pela presença do banho térmico [26]. Nesse caso o objeto que caracteriza a quebra de simetria de Lorentz (análogo a $\theta^{\mu \nu}$ ) é a velocidade do banho térmico $u^{\mu}$.

No entanto, a introdução deste produto em (3.45), não permite que tenhamos invariância sob transformações de coordenadas, uma vez que a relação de comutação (4.3) não foram consistente com a equação (4.2). No entanto, é possível uma simétria local sob uma classe de transformações infinitesimais restritas, através da utilização de um parâmetro que chamamos de $\tilde{w}(x)$. Pelas equações (4.6) e (4.7b) encontramos a equação diferencial (4.9) que possui solução não trivial que nos leva a forma do parâmetro infinitesimal restrito $\tilde{w}(x)$. Este resultado nos conduziu a invariância da relação de comutação em (4.16). 

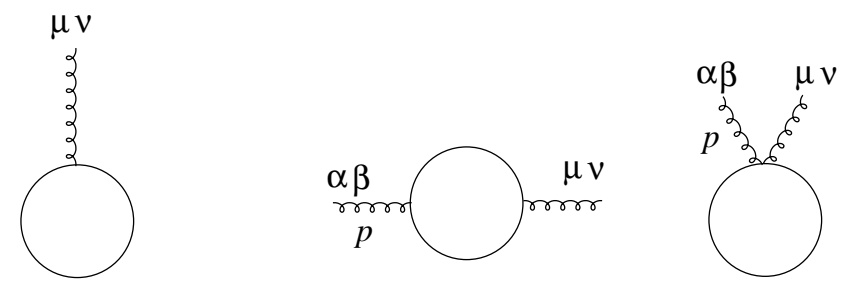

Figura 5.1:

O mais interessante neste desenvolvimento, foram as consequências geradas por essa transformação restrita.

A primeira dessas consequências é a preservação do elemento de volume $\mathrm{d}^{4} x^{\prime}=\mathrm{d}^{4} x$, conhecida como teoria unimodular da gravitação. A segunda, permitiu eliminarmos o termo extra do Jacobiano na transformação do determinante da métrica, fazendo com que a variação (4.20) fosse nula, uma vez que $\sqrt{-g}$ possui um valor constante, já que a métrica satisfaz a condição de matriz unimodular. A terceira e principal consequência permitiu encontrarmos a forma da ação não comutativa até primeira ordem em $h$.

É interessante observar que o resultado que obtivemos na primeira ordem em $h_{\mu \nu}$ envolve apenas o anti-comutador Moyal entre as derivadas do campo escalar. Disso segue imediatamente que a expansão em potências de $\theta^{\mu \nu}$ não possui o termo linear em $\theta^{\mu \nu}$. Esse resultado está de acordo com o que foi notado recentemente em [24].

Finalmente, embora a técnica que utilizamos forneça apenas a expansão de campos fracos (não saberíamos determinar a forma fechada da ação), podemos em princípio determinar iterativamente os termos subsequentes. Isso é ilustrado no apêndice, para o caso comutativo, utilizando computação simbólica. A partir do termo de primeira ordem podemos usar novamente a identidade de Ward e determinar o termo seguinte. Desse modo, teremos os dois vértices de interação entre o gráviton e bosons escalares, necessários para o cálculo de processos tais como as correções quânticas para o tensor de energia momento e de auto-energia mostradas na figura (5.1). 


\section{Apêndice A}

\section{Breve revisão sobre teorias de gauge}

Consideremos inicialmente a Lagrangiana de Dirac para férmions livres de massa $m$

$$
\mathcal{L}^{(0)}=i \bar{\psi} \gamma^{\mu} \partial_{\mu} \psi-m \bar{\psi} \psi
$$

A fim de descrever fenômenos de interação entre os férmions teríamos que adicionar um termo de interação a $\mathcal{L}^{(0)}$, assumindo, da maneira usual, que a interação é mediada por um outro campo. No entanto, há uma infinidade de possibilidades, compatíveis com a invariância de Lorentz, para um tal termo de interação.

Além da simetria sob transformações de Lorentz, a lagrangiana $\mathcal{L}^{(0)}$ é também invariante sob a seguinte transformação de gauge global

$$
\psi \rightarrow \mathrm{e}^{i \omega} \psi
$$

onde $\omega$ é um número real. Esta simetria global também não é suficiente para restringir a forma de um possível termo de interação.

Vejamos o que ocorre quando impomos o princípio de invariância local fazendo $\omega \rightarrow$ $\omega(x)$. Obviamente, a lagrangiana livre (A.1) não é invariante sob transformações locais e se modifica como

$$
\mathcal{L}^{(0)} \rightarrow \mathcal{L}^{(0)}-\bar{\psi} \gamma^{\mu} \psi \partial_{\mu} \omega
$$

Neste ponto, podemos observar que a grandeza proporcional à $\partial_{\mu} \omega$ pode ser cancelada se adotarmos o seguinte procedimento. Primeiro adicionamos à lagrangiana livre um termo 
de interação envolvendo um campo vetorial $A_{\mu}$ de tal forma que a lagrangiana total (livre mais interação) é dada por

$$
\mathcal{L}_{\text {mat }}=i \bar{\psi} \gamma^{\mu} \partial_{\mu} \psi-m \bar{\psi} \psi+\bar{\psi} \gamma^{\mu} \psi A_{\mu}
$$

Em seguida impomos a lei de transformação de $A_{\mu}$ como sendo ${ }^{1}$

$$
A_{\mu} \rightarrow A_{\mu}+\partial_{\mu} \omega
$$

Vemos assim que o princípio de invariância local traz consigo uma previsão fenomenológica bem determinada, a saber, a forma da interação entre os campos da teoria. Temos uma teoria envolvendo espinores a campos vetoriais interagentes, cujas leis de transformação são dadas pelas equações (A.2) (com $\omega$ dependente das coordenadas de espaço-tempo) e (A.5). Resta saber qual é a dinâmica clássica de $A_{\mu}(x)$.

Mencionamos anteriormente que a simetria local pode ser implementada substituindo a derivada usual por uma derivada co-variante $D_{\mu}$. É imediato verificar que, de fato, a lagrangiana (A.4) é obtida de (A.1) substituindo $\partial_{\mu}$ por

$$
D_{\mu}=\partial_{\mu}-i A_{\mu}
$$

Além da simplicidade formal, o procedimento $\partial_{\mu} \rightarrow D_{\mu}$ nos indica um possível caminho para estabelecer a conexão com a forma de lagrangiana de Maxwell. Levando em conta que o tensor de campo eletromagnético $F_{\mu \nu}$ é anti-simétrico nos índices de Lorentz é natural supor que este possa ser escrito em termos do comutador das derivadas co-variantes $\left[D_{\mu}, D_{\nu}\right]$. De fato, podemos verificar facilmente que

$$
F_{\mu \nu}=-i\left[D_{\mu}, D_{\nu}\right]=\partial_{\mu} A_{\nu}-\partial_{\nu} A_{\mu}-i\left[A_{\mu}, A_{\nu}\right]
$$

Naturalmente, o último termo, envolvendo o comutador dos campos de gauge $\left[A_{\mu}, A_{\nu}\right]$ é identicamente nulo no caso da teoria de Maxwell. Mas a generalização para o caso não-abeliano torna-se imediata a partir da equação (A.7).

\footnotetext{
${ }^{1}$ As equações (A.5) e (A.2) correspondem às equações (5) e (9) do artigo de Fock em [2].
} 
A lagrangiana que descreve a dinâmica dos campos de gauge (lagrangiana de Maxwell) é dada por ${ }^{2}$

$$
\mathcal{L}_{\text {Maxwell }}=-\frac{1}{4} F_{\mu \nu} F^{\mu \nu}
$$

onde $F^{\mu \nu}$ é dado por (A.7), sem o termo $\left[A_{\mu}, A_{\nu}\right]$. Podemos observar que $\mathcal{S}_{\text {Maxwell }}$ é a forma mais simples compatível a invariância de Lorentz. É importante ressaltar que a invariância de gauge e de Lorentz ainda permite outros tipos de termos na lagrangiana. Dentre as possibilidades podemos mencionar termos envolvendo o tensor totalmente antisimétrico $\epsilon^{\mu \nu \alpha \beta}$, "potências" maiores de $F_{\mu \nu}$, ou mesmo termos de Chern-Simons $[27,28]$ quando a dimensão do espaço tempo for ímpar. No presente caso, temos uma teoria de campos definida pela lagrangiana da QED

$$
\mathcal{L}_{\mathrm{QED}}=\mathcal{L}_{\text {Maxwell }}+\mathcal{L}_{\text {mat }}
$$

onde $\mathcal{L}_{\text {Maxwell }}$ e $\mathcal{L}_{\text {mat }}$ são respectivamente dadas por (A.8) e (A.4), que é invariante sob as transformações locais (A.2) e (A.5).

Quando o termo $\left[A_{\mu}, A_{\nu}\right]$ em (A.7) é não nulo temos uma teoria não-abeliana de gauge. Existem diversos cenários nos quais essa possibilidade pode ser realizada na natureza. Por exemplo, na QCD ou na teoria eletrofraca ou mesmo no contexto mais atual das teorias de campos formuladas em espaços não comutativos [15]. Tanto no caso da QCD como na teoria eletrofraca o grupo $S U(N)(N=3$ para a QCD e $N=2$ para a teoria eletrofraca) desempenha um papel fundamental como o grupo de transformações não-abelianas.

A grandeza $N$ representa o número de componentes do espinor de Dirac no espaço de simetria interna (Yang e Mills haviam proposto um dubleto de isospin formado pelos estados p (próton) e n (nêutron) e transformando-se sob o grupo $S U(2))$. Suponhamos agora que os espinores em (A.1) são objetos de $N$ componentes, $\psi=\psi_{1}, \psi_{2}, \cdots, \psi_{N}$, e se transformam como

$$
\psi \rightarrow U \psi
$$

onde $U$ é um elemento do grupo $S U(N)$. Como $\bar{\psi} \rightarrow \bar{\psi} U^{\dagger}$ e $U^{\dagger} U=1$ a lagrangiana

\footnotetext{
${ }^{2} \mathrm{O}$ sinal negativo na lagrangiana de Maxwell leva a um coeficiente positivo para $\left(\partial_{0} A_{i}\right)^{2}$, ou seja, quanto maior for a variação temporal maior será a ação.
} 
livre é invariante quando $U$ não depende das coordenadas do espaço tempo. Impondo a condição de localidade das transformações, $(U \rightarrow U(x))$ vemos que

$$
\partial_{\mu} \psi \rightarrow \partial_{\mu}(U \psi)=U \partial \psi+\left(\partial_{\mu} U\right) \psi=U\left[\partial_{\mu} \psi+\left(U^{\dagger} \partial_{\mu} U\right) \psi\right]
$$

A invariância é recuperada fazendo a substituição $\partial_{\mu} \psi \rightarrow D_{\mu} \psi$ onde $D_{\mu}$ é dada pela equação (A.6) com $A_{\mu}$ não-abeliano. Porém agora o potencial $A_{\mu}$ deve ser transformar de tal forma que a condição de co-variância

$$
D_{\mu} \psi \rightarrow U D_{\mu} \psi
$$

seja satisfeita. Usando (A.10), (A.11) e (A.6) a equação (A.12) nos dá

$$
A_{\mu} \rightarrow U A_{\mu} U^{\dagger}-i\left(\partial_{\mu} U\right) U^{\dagger}=U\left(A_{\mu}+i \partial_{\mu}\right) U^{\dagger}
$$

A última igualdade decorre da condição $U U^{\dagger}=1$ que por sua vez permite escrever $\left(\partial_{\mu} U\right) U^{\dagger}=-U\left(\partial_{\mu} U^{\dagger}\right)$

Podemos agora inferir algumas importantes propriedades satisfeitas pelos potenciais não abelianos $A_{\mu}$. Obviamente, tendo em vista a equivalência física dos potenciais relacionados por (A.13), cada $A_{\mu}$ tem que ser uma matriz $N$ por $N$, como é o caso de $U$. Além disso, essas matrizes podem ser tomadas consistentemente como hermitianas, uma vez que (A.13) preserva a condição $A_{\mu}-A_{\mu}^{\dagger}=0$. Escrevendo os elementos do grupo $S U(N)$ explicitamente em termos dos geradores hermitianos $T^{a}$ como

$$
U=\mathrm{e}^{i \omega^{a} T^{a}}
$$

e considerando as transformações com $\omega^{a}$ infinitesimal $U \simeq 1+i \omega^{a} T^{a}$, obtemos a partir de (A.13)

$$
A_{\mu} \rightarrow A_{\mu}+\partial_{\mu} \omega^{a} T^{a}+i \omega^{a}\left[T^{a}, A_{\mu}\right] .
$$

Tomando o traço de (A.15) e usando $\operatorname{tr} T^{a}=0$ bem como a propriedade cíclica no termo envolvendo o comutador, vemos que o traço de $A_{\mu}$ não se transforma. Portanto, podemos tomar $A_{\mu}$ consistentemente como matrizes de traço nulo.

As duas propriedades acima verificadas, a saber, hermiticidade e traço nulo, nos permitem expressar os potenciais não abelianos em termos dos geradores $T^{a}$ da seguinte 
maneira

$$
A_{\mu}=A_{\mu}^{a} T^{a}
$$

onde $A_{\mu}^{a}$ são grandezas reais. Logo, em uma teoria não abeliana de gauge, existem tantos potenciais quantos são os geradores do grupo $S U(N)$, ou seja, $N^{2}-1$ potenciais (8 glúons no caso da QCD). Substituindo em (A.15) a álgebra de Lie dos geradores

$$
\left[T^{a}, T^{b}\right]=i f^{a b c} T^{c}
$$

( $f^{a b c}$ são as constantes de estrutura do grupo) obtemos a seguinte transformação para os potenciais $A_{\mu}^{a}$

$$
A_{\mu}^{a} \rightarrow A_{\mu}^{a}+D_{\mu}^{a b} \omega^{b}
$$

onde

$$
D_{\mu}^{a b} \equiv \delta^{a b} \partial_{\mu}-f^{a b c} A_{\mu}^{c}
$$

é o operador de derivada co-variante na representação adjunta. É interessante observar que para $\omega$ independente das coordenadas de espaço-tempo os potenciais $A_{\mu}^{a}$ se transformam como membros da representação adjunta do grupo (são submetidos à uma "rotação" infinitesimal no espaço de dimensão $N^{2}-1$ ).

Usando (A.13) podemos facilmente verificar que o tensor $F_{\mu \nu}$ em (A.7) se transforma como

$$
F_{\mu \nu} \rightarrow U F_{\mu \nu} U^{\dagger}
$$

Desta relação segue imediatamente que a grandeza $\operatorname{tr} F_{\mu \nu} F^{\mu \nu}$ é invariante sob as transformações locais de $S U(N)$. Portanto, a versão não abeliana da lagrangiana de Maxwell pode ser escrita como

$$
\mathcal{L}_{\text {Maxwell }}^{\text {na }}=-\frac{1}{2} \operatorname{tr} F_{\mu \nu} F^{\mu \nu}
$$

Substituindo a relação (A.16) em (A.7) e usando (A.17), podemos obter a forma explícita das componentes $F_{\mu \nu}^{a}$ de $F_{\mu \nu}=F_{\mu \nu}^{a} T^{a}$ como sendo dadas por

$$
F_{\mu \nu}^{a}=\partial_{\mu} A_{\nu}^{a}-\partial_{\nu} A_{\mu}^{a}+f^{a b c} A_{\mu}^{b} A_{\nu}^{c} .
$$


Usando (A.18), bem como a relação ${ }^{3}$

$$
f^{a b e} f^{c d e}+f^{b c e} f^{a d e}+f^{b d e} f^{c a e}=0,
$$

podemos verificar facilmente que

$$
F_{\mu \nu}^{a} \rightarrow F_{\mu \nu}^{a}-f^{a b c} \omega^{b} F_{\mu \nu}^{c}
$$

Ou seja, $F_{\mu \nu}^{a}$ transforma-se como um membro da representação adjunta.

Levando em conta a normalização

$$
\operatorname{tr} T^{a} T^{b}=\frac{1}{2} \delta^{a b}
$$

a lagrangiana (A.21) pode então ser reescrita em termos de $F_{\mu \nu}^{a}$ como

$$
\mathcal{L}_{\text {Maxwell }}^{\text {na }}=-\frac{1}{4} F_{\mu \nu}^{a} F^{a \mu \nu}
$$

Temos assim, sumarizando, uma teoria definida pela lagrangiana

$$
\mathcal{L}^{\text {na }}=\mathcal{L}_{\text {Maxwell }}^{\text {na }}+\mathcal{L}_{\text {mat }}^{\text {na }}
$$

onde $\mathcal{L}_{\text {Maxwell }}^{\text {na }} \mathcal{L}_{\text {mat }}^{\text {na }}$ são dadas respectivamente por (A.26) e (A.4), considerando, nesta última, $\psi$ como um espinor de $N$ componentes internas. A teoria de campos clássica assim definida é invariante sob as transformações (A.10) e (A.18) (note que a invariância local da QED, segue como um caso particular tomando $f^{a b c}=0$ e $\left.\omega^{a}=\omega\right)$.

\section{A.1 Quantização e regras de Feynman}

A quantização perturbativa de teorias de campo pode ser formalmente sumarizada em termos de integrais de trajetória através da seguinte relação

$$
\begin{aligned}
Z[J] & \equiv \int D \phi \mathrm{e}^{i S[\phi]+i J \cdot \phi}=\int D \phi \mathrm{e}^{-\frac{1}{2} \phi \cdot K \cdot \phi+i V(\phi)+i J \cdot \phi} \\
& =\mathrm{e}^{V\left(\frac{\delta}{i \delta J}\right)} \mathrm{e}^{\frac{1}{2} \cdot J \frac{1}{K} \cdot J} \cdot
\end{aligned}
$$

\footnotetext{
${ }^{3}$ Esta relação segue da identidade de Jacobi $\left[T^{a},\left[T^{b}, T^{c}\right]\right]+$ permut. cíclicas $=0$.
} 
Do lado esquerdo temos o funcional gerador das funções de Green de uma teoria de campos genérica. O campo $\phi$ representa aqui um "vetor" cujas componentes $\phi_{i}$ encapsulam as coordenadas de espaço-tempo, índices de Lorentz, índices de simetria interna (cor), etc. O "produto escalar" "." em (A.28) representa uma soma sobre índices de Lorentz, índices internos e também as coordenadas de espaço-tempo, ou seja, uma integral. A matriz formal $K$ define o termo quadrático da teoria e a grandeza $V(\phi)$ representa todos os termos envolvendo potências de $\phi$ maiores do que dois. Estas grandezas podem ser lidas diretamente na lagrangiana da teoria.

A passagem da primeira para a segunda linha de (A.28) é feita via cálculo de integrais gaussianas. Funções de Green são então obtidas por derivação funcional em relação à $J$, ou seja, aplicando o operador $\delta / \delta J$ no lado direito de (A.28). Este algoritmo produz expressões para as funções de Green que podem ser sinteticamente representadas em termos dos diagramas de Feynman da teoria os quais descrevem processos físicos de propagação e interação (produzindo espalhamento ou decaimento) dos quanta associados aos campos $\phi$ (partículas). Este é o único significado que atribuiremos à expressões como (A.28), ou seja, um algoritmo bastante direto que permite conectar a ação $S[\phi]$ com as regras de Feynman da teoria quântica. Na prática, freqüentemente os problemas são primeiramente reconhecidos e resolvidos via formalismo diagramático e só depois formulados elegantemente na linguagem de integrais funcionais. De fato, foi dessa forma que Feynman [29] descobriu que a teoria quântica não pode ser consistentemente definida utilizando simplesmente a ação clássica de Yang-Mills (além de um termo de fixação de gauge como na QED) em (A.28).

Ao tentarmos aplicar a relação (A.28) no caso da teoria de Yang-Mills, nos deparamos de imediato com um problema fundamental, a saber, $K$ não possui inversa. De fato, podemos verificar facilmente que a matriz $K$ correspondente à expressão (A.21) é proporcional ao operador

$$
Q_{\text {transv. }}^{\mu \nu} \equiv \eta^{\mu \nu} \partial^{2}-\partial^{\mu} \partial^{\nu}
$$

Notando que

$$
\partial_{\nu} \omega(x) Q_{\text {transv. }}^{\mu \nu}=0
$$


ou seja, $Q_{\text {transv }}^{\mu \nu}$ possui auto-valores nulos, concluímos que a matriz $K^{-1}$ não existe no caso da teoria de Yang-Mills. Ao nível clássico esta característica é bem conhecida na teoria de Maxwell, onde sabemos que a solução da equação $\partial_{\mu} F^{\mu \nu}=J^{\nu}$ ou, equivalentemente, $Q_{\mu \nu} A^{\nu}=J^{\nu}$, somente pode ser obtida fazendo uma escolha de gauge para os potenciais $A_{\mu}$. Ou seja, há vários (uma infinidade) potenciais correspondentes a um mesmo sistema físico.

Este problema torna-se mais crítico no caso das teorias não-abelianas e constituiu um importante obstáculo para que a teoria de Yang-Mills pudesse ser aceita, tendo em vista a dificuldade de se obter regras de Feynman e realizar cálculos perturbativos. Progressos neste direção foram também importantes para as primeiras tentativas de se quantizar a gravitação. No início dos anos 1960, Feynman observou que seria necessário introduzir campos fictícios (fantasmas) a fim de cancelar uma aparente violação de unitariedade [29]. Esse procedimento foi depois confirmado por DeWitt em cálculos mais detalhados [30]. Mais tarde, Faddeev e Popov [31] desenvolveram um método sistemático para lidar com este tipo de problema, ao nível quântico, no contexto de integrais de trajetória, ou seja, na formulação sintetizada em (A.28), bem como esclareceram o papel dos fantasmas. Esse método é essencial no caso da teoria de Yang-Mills e também na gravitação (em geral, em teorias de gauge não lineares).

A fim de ilustrar os principais aspectos do método, vamos considerar a expressão do lado esquerdo de (A.28) com $J=0$. Além disso, vamos focalizar apenas a teoria pura de Yang-Mills (sem os férmions) que é onde está o problema. Neste caso, a grandeza relevante é

$$
Z[0]=\int D A \mathrm{e}^{i S_{\text {Max. }}^{\text {na }}[A]} ; \quad S_{\text {Max. }}^{\text {na }}[A]=\int \mathrm{d}^{4} x \mathcal{L}_{\text {Maxwell }}^{\text {na }},
$$

onde $\mathcal{L}_{\text {Maxwell }}^{\text {na }}$ está dada em (A.26). Primeiramente, podemos verificar que a medida de integração $D A$ em (A.31), da mesma forma que a ação $S_{\text {Max. }}^{\text {na }}[A]$, também é invariante. De fato, sob uma transformação de gauge $A \rightarrow A^{\omega}$ teremos

$$
D A \rightarrow D A \operatorname{det}\left(\frac{\partial A_{\mu}^{\omega a}}{\partial A_{\nu}^{b}}\right)=D A \operatorname{det}\left(\delta^{a b}-f^{a b c} \omega^{c}\right)=D A\left(1+\mathcal{O}\left(\omega^{2}\right)\right),
$$


onde utilizamos (A.18) e a identidade

$$
\operatorname{det}(1+L)=\mathrm{e}^{\operatorname{tr} \log (1+L)}=1+\operatorname{tr} L+\mathcal{O}\left(L^{2}\right) .
$$

Podemos então reformular o problema de quantização de uma teoria de gauge da seguinte maneira. Temos que calcular uma integral cujo integrando permanece constante em um sub-espaço das variáveis de integração. Assim o problema consiste em fatorizar explicitamente a integração sobre as chamadas órbitas de gauge, de maneira análoga à fatorização de $(2 \pi)$ que ocorre em uma integral do tipo $\int \mathrm{d} x \mathrm{~d} y \exp \left[S\left(x^{2}+y^{2}\right)\right]$ quando utilizamos coordenadas polares ( $2 \pi$ é o "volume" do grupo de rotações em duas dimensões). Como veremos, ao separarmos este fator, teremos, ao mesmo tempo, resolvido o problema de definir o termo quadrático da ação, uma vez que a matriz $K$ em (A.28) será modificada. Seguindo o procedimento de Faddeev e Popov, definimos inicialmente a grandeza $\Delta[A]$ através da relação

$$
1=\Delta[A] \int D \omega \delta\left[f\left(A^{\omega}\right)\right]
$$

onde $f\left(A^{\omega}\right)$ é uma grandeza dependente de gauge e $\delta$ é o funcional delta de Dirac. Como a integração é feita sobre todo o espaço de gauge, é imediato verificar que $\Delta[A]$ é independente de gauge. Inserindo (A.34) no integrando de (A.31), teremos

$$
Z[0]=\int D \omega \int D A \mathrm{e}^{i S_{\text {Max. }}^{\text {na }}[A]} \Delta[A] \delta\left[f\left(A^{\omega}\right)\right] .
$$

Fazendo a transformação inversa $A \rightarrow A^{\omega^{-1}}$ e usando a invariância de $D A, S_{\text {Max. }}^{\text {na }}[A]$ e $\Delta[A]$ obtemos finalmente

$$
Z[0]=\left(\int D \omega\right) \int D A \Delta[A] \delta[f(A)] \mathrm{e}^{i S_{\text {Max. }}^{\text {na }}[A]}
$$

Embora o volume de um grupo compacto seja finito, a integração $\left(\int D \omega\right)$ é infinita, uma vez que há um elemento do grupo para cada ponto do espaço-tempo. A definição da teoria quântica é feita eliminando este fator infinito do funcional gerador. Equivalentemente, estamos introduzindo um ansatz segundo o qual a medida invariante na integração funcional (mesmo com $J \neq 0$ ) deve ser substituída por $D A \Delta[A] \delta[f(A)]$.

A função $f(A)$ define o tipo de fixação de gauge utilizado para quantizar a teoria. Usualmente ela é tomada como sendo linear em $A$ da seguinte forma

$$
f(A)=G^{\mu} A_{\mu}^{a}-\sigma^{a}
$$


onde $G^{\mu}$ é um operador ou um vetor qualquer e $\sigma^{a}$ é uma função qualquer das coordenadas de espaço-tempo. Por exemplo, a escolha usual na QED é o chamado gauge co-variante onde $G^{\mu}=\partial^{\mu}$. A forma explícita de $\Delta[A]$ pode agora ser facilmente obtida. Substituindo (A.37) em (A.34), teremos

$$
\Delta[A]=\left(\int D \omega \delta\left[G^{\mu} A_{\mu}^{a}-\sigma^{a}\right]\right)^{-1} .
$$

Em seguida, levamos em conta que $\Delta[A]$ sempre aparece multiplicado por $\delta\left[G^{\mu} A_{\mu}^{a}-\sigma^{a}\right]$ e fazemos a transformação (A.18), obtendo

$$
\Delta[A]=\left(\int D \omega \delta\left[G^{\mu}\left(f^{a b c} \omega^{b} A_{\mu}^{c}-\partial_{\mu} \omega^{a}\right)\right]\right)^{-1}+\cdots,
$$

onde $\cdots$ representa os termos que não contribuem quando multiplicados por $\delta\left[G^{\mu} A_{\mu}^{a}-\sigma^{a}\right]$. Definindo o operador $K^{a b}(x, y)$ tal que

$$
\int \mathrm{d}^{4} y K^{a b}(x, y) \omega^{b}(y)=G^{\mu}\left(f^{a b c} \omega^{b} A_{\mu}^{c}-\partial_{\mu} \omega^{a}\right)
$$

e usando a identidade

$$
\int D \omega \delta[K \cdot \omega]=(\operatorname{det} K)^{-1}
$$

obtemos

$$
\Delta[A]=\operatorname{det} K=\int D c_{1} D c_{2} \mathrm{e}^{i S_{\text {ghost }}\left[c_{1}, c_{2}\right]}
$$

onde

$$
S_{\text {ghost }}\left[c_{1}, c_{2}\right]=\int \mathrm{d}^{4} x \mathrm{~d}^{4} y c_{1}^{a}(x) K_{a b}(x, y) c_{2}^{b}(y)
$$

e $c_{i}(x), i=1,2$ são variáveis de Grassmann conhecidas como fantasmas de Faddeev Popov. A denominação "fantasmas" é devida ao fato de que esses campos violam a conexão spinestatística, tendo em vista que, embora sejam campos escalares, eles anti-comutam. Esta violação é aceitável uma vez que tais campos não estão associados com partículas físicas. Na teoria de campos à temperatura finita somos levados à atribuir uma estatística térmica de Bose-Einstein para os fantasmas.

Usando a forma explícita de $K^{a b}$ que pode ser derivada a partir de (A.40), obtemos

$$
S_{\text {ghost }}\left[c_{1}, c_{2}\right]=-\int \mathrm{d}^{4} x c_{1}^{a}(x) G^{\mu}\left(\delta^{a b} \partial_{\mu}-f^{a b c} A_{\mu}^{c}\right) c_{2}^{b}(x) .
$$


Em termos do operador de derivada co-variante em (A.19), teremos

$$
S_{\text {ghost }}\left[c_{1}, c_{2}\right]=-\int \mathrm{d}^{4} x c_{1}^{a}(x) G^{\mu} D_{\mu}^{a b} c_{2}^{b}(x)
$$

É possível também obter uma forma exponenciada para $\delta[f(A)]$. Levando em conta que a integral funcional em (A.36) não depende de $f(A)$ e, em particular, não depende de $\sigma^{a}$, podemos multiplica-la por um funcional qualquer de $\sigma$ e integrar sobre $\sigma$. Uma escolha conveniente é

$$
\mathrm{e}^{-\frac{i}{2 \xi} \int \mathrm{d}^{4} x \sigma(x)^{2}}
$$

onde $\xi$ é uma constante, e usando o funcional delta de Dirac, teremos

$$
Z=\int D A D c_{1} D c_{2} \mathrm{e}^{i S_{\text {Max. }}^{\text {na }}+i S_{\text {ghost }}+i S_{\text {fix }}}
$$

onde

$$
S_{\text {fix }}=-\frac{1}{2 \xi} \int \mathrm{d}^{4} x f(A)^{2}=-\frac{1}{2 \xi} \int \mathrm{d}^{4} x\left(G^{\mu} A_{\mu}^{a}\right)^{2}
$$

Podemos notar que ocorrerá uma grande simplificação no caso da teoria abeliana. De fato, tomando $f^{a b c}=0$, vemos que o campo de fantasmas se desacopla dos campos de gauge e a integração funcional sobre $c_{1}$ e $c_{2}$ resulta em um fator constante que pode ser omitido ${ }^{4}$. Naturalmente, estamos supondo que $G^{\mu}$ depende linearmente dos campos de gauge. Algumas escolhas mais populares para $G^{\mu}$ são as seguintes:

- Gauge de Coulomb: $G^{\mu}=(0, \vec{\partial})$.

- Gauge de Lorentz: $G^{\mu}=\partial^{\mu}$.

- Gauge Axial: $G^{\mu}=n^{\mu}$, onde $n^{\mu}$ define uma direção fixa no espaço-tempo.

- Gauge temporal: $G^{\mu}=(1, \overrightarrow{0})$.

Em cada um destes casos, o parâmetro $\xi$, introduzido em (A.46), pode ser variado produzindo toda uma classe de gauges .

\footnotetext{
${ }^{4}$ No entanto, este fator possui um papel importante na contagem dos graus de liberdade da QED formulada a temperatura finita.
} 
Podemos agora retornar à relação (A.28) considerando seguinte forma para a ação

$$
S\left[A, c_{1}, c_{2}\right]=S_{\text {Max. }}^{\text {na }}+S_{\text {ghost }}+S_{\text {fix }}
$$

Vemos que o termo $S_{\text {fix }}$ modifica o termo quadrático em $S_{\text {Max. }}^{\text {na }}$, de tal forma que agora o propagador da teoria pode ser obtido. Por exemplo, no caso do gauge de Lorentz, teremos, após integração por partes

$$
S\left[A, c_{1}, c_{2}\right]=\int \mathrm{d}^{4} x\left(\frac{1}{2} A_{\mu}^{a} Q_{a b}^{\mu \nu} A_{\nu}^{b}+c_{1}^{a} Q_{a b}^{\text {ghost }} c_{2}^{b}+\mathcal{L}^{\text {int. }}\right),
$$

onde os termos de interação em $\mathcal{L}^{\text {int. }}$ serão explicitados mais adiante e

$$
Q_{a b}^{\text {ghost }}=-\delta_{a b} \partial^{2}
$$

O novo operador quadrático

$$
Q_{a b}^{\mu \nu} \equiv \delta_{a b}\left[\eta^{\mu \nu} \partial^{2}-\left(1-\frac{1}{\xi}\right) \partial^{\mu} \partial^{\nu}\right]=\delta_{a b}\left(Q_{\text {transv. }}^{\mu \nu}-\frac{1}{\xi} \partial^{\mu} \partial^{\nu}\right)
$$

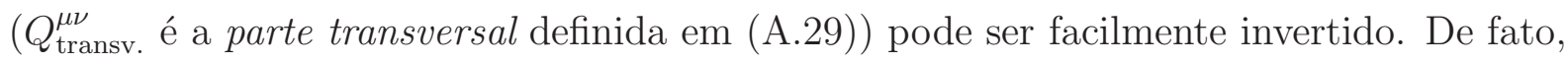
a equação

$$
Q_{a d}^{\mu \alpha} D_{\alpha \nu}^{d b}(x-y)=\delta_{\nu}^{\mu} \delta_{a}^{b} \delta^{4}(x-y)
$$

tem a seguinte solução

$$
D_{a b}^{\mu \nu}(x-y)=i \int \frac{\mathrm{d}^{4} k}{(2 \pi)^{4}} \tilde{D}_{a b}^{\mu \nu}(k) \mathrm{e}^{i k(x-y)},
$$

onde

$$
i \tilde{D}_{a b}^{\mu \nu}(k) \equiv-i \frac{\delta_{a b}}{k^{2}+i \epsilon}\left[\eta^{\mu \nu}-(1-\xi) \frac{k^{\mu} k^{\nu}}{k^{2}}\right]
$$

é o propagador livre no espaço dos momentos. O parâmetro $\epsilon$, implícito na integral (A.47), é infinitesimal e positivo, de tal forma a tornar a integração funcional convergente para grandes valores do integrando. Estamos também levando em conta o fator $(i)$ presente na exponencial em (A.47) (este procedimento será consistentemente adotado mais adiante quando forem definidas as regras de Feynman para o propagador dos fantasmas e para as interações). De maneira semelhante, os propagadores de férmion e de fantasmas são obtidos respectivamente de (A.1) e (A.51), sendo dados por

$$
i \tilde{S}(k) \equiv \frac{i}{\not k-m+i \epsilon}=i \frac{\not k+m}{k^{2}-m^{2}+i \epsilon}
$$


e

$$
i \tilde{D}_{a b}^{\text {ghost }} \equiv \frac{i}{k^{2}+i \epsilon} \delta_{a b}
$$

A fim de obter as regras de Feynman para os vértices da teoria, vamos inicialmente escrever a lagrangiana de interação $\mathcal{L}^{\text {int }}$. Colecionando os termos envolvendo interações entre os férmions, os campos de gauge e os fantasmas, presentes nas expressões (A.4), (A.26) e (A.43), teremos

$$
\begin{aligned}
\mathcal{L}^{\mathrm{int}} & =g \bar{\psi} \gamma^{\mu} A_{\mu}^{a} T^{a} \psi-\frac{1}{2} g f^{a b c}\left(\partial_{\mu} A_{\nu}^{a}-\partial_{\nu} A_{\mu}^{a}\right) A^{b \mu} A^{c \nu} \\
& -\frac{1}{4} g^{2} f^{e a b} f^{e c d} A_{\mu}^{a} A_{\nu}^{b} A^{c \mu} A^{d \nu}+g f^{a b c} c_{1}^{a} c_{2}^{b} G_{\mu} A^{c \mu}
\end{aligned}
$$

Note que estamos introduzindo explicitamente a constante de acoplamento $g$, fazendo $A \rightarrow g A$ e $\mathcal{L} \rightarrow \mathcal{L} / g^{2}$. Levando em conta a simetria bosônica dos campos de gauge e as propriedades de ciclicidade e de anti-simetria das constantes de estrutura $\left(f^{a b c}=f^{b c a}=\right.$ $f^{c a b}$ e $\left.f^{a b c}=-f^{a c b}\right)$, a expressão para $i S^{\text {int }}=i \int \mathrm{d}^{4} x \mathcal{L}^{\text {int }}$ gera os seguintes vértices no espaço dos momentos

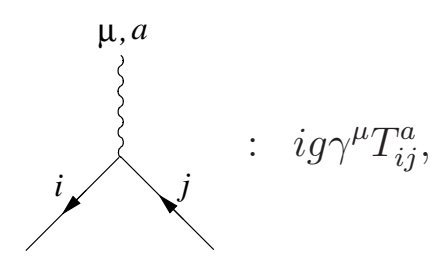

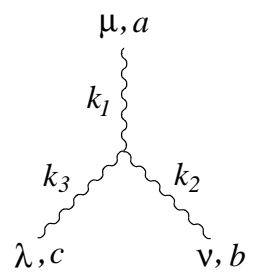

$$
\text { : } g f^{a b c}\left[\left(k_{1}-k_{2}\right)^{\lambda} \eta^{\mu \nu}+\left(k_{2}-k_{3}\right)^{\mu} \eta^{\nu \lambda}+\right.
$$

$$
\left.\left(k_{3}-k_{1}\right)^{\nu} \eta^{\lambda \mu}\right]
$$




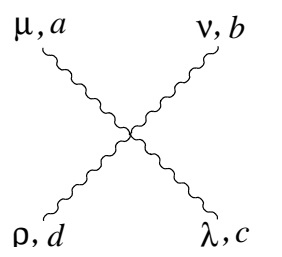

$$
: \quad-i g^{2}\left[f^{a b e} f^{c d e}\left(\eta^{\mu \lambda} \eta^{\nu \rho}-\eta^{\mu \rho} \eta^{\nu \lambda}\right)\right.
$$$$
+f^{a d e} f^{c b e}\left(\eta^{\mu \lambda} \eta^{\nu \rho}-\eta^{\mu \nu} \eta^{\lambda \rho}\right)
$$

$$
\left.+f^{a c e} f^{b d e}\left(\eta^{\mu \nu} \eta^{\lambda \rho}-\eta^{\mu \rho} \eta^{\nu \lambda}\right)\right]
$$

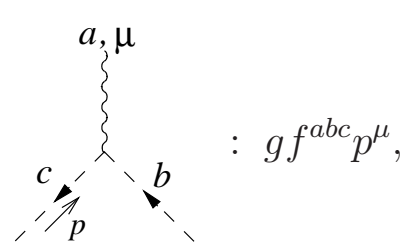

onde está implícito um fator de conservação de momento $(2 \pi)^{4} \delta(\Sigma k)^{5}$. Todos os momentos são tomados entrando nos vértices. Cada um dos propagadores é representado da maneira usual pelos seguintes diagramas

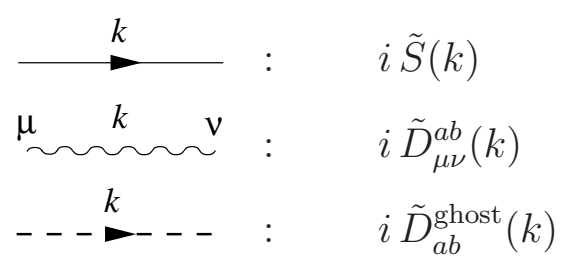

Vimos que a definição da teoria quântica de gauge requer a introdução de termos adicionais na ação de Yang-Mills envolvendo os fantasmas de Faddeev-Popov. Devido ao acoplamento entre os fantasmas e os campos de gauge, as transformações de gauge usuais em (A.10) e (A.18) não são uma simetria da ação modificada em (A.49). Somos então levados naturalmente a supor que os campos de fantasmas também devem se transformar de maneira a compensar a transformação dos campos de gauge. A fim de examinar esta possibilidade, vamos supor que o parâmetro infinitesimal da transformação de gauge possa ser escrito em termos dos campos de fantasmas $c_{i}^{a}(x)$, da seguinte maneira

$$
\omega^{a}(x)=(\delta \lambda) c_{2}^{a}(x)
$$

\footnotetext{
${ }^{5}$ Fatores de simetria serão levados em conta no cálculo explícito das amplitudes quânticas.
} 
A grandeza $\delta \lambda$ é uma variável de Grassmann $\left((\delta \lambda)^{2}=0\right)$ independente das coordenadas de espaço-tempo e que satisfaz a relação de anti-comutação

$$
\left\{(\delta \lambda), c_{2}^{a}(x)\right\}=0
$$

Note que $\delta \lambda$ desempenha o importante papel de manter o caráter bosônico do parâmetro $\omega$. Em termos do ansatz (A.61), as transformações de gauge locais (A.10) e (A.18) podem ser reescritas como

$$
\begin{gathered}
\delta \psi=i(\delta \lambda) T^{a} c_{2}^{a} \psi, \\
\delta A_{\mu}^{a}=(\delta \lambda) D_{\mu}^{a b} c_{2}^{b} .
\end{gathered}
$$

A transformação (A.64) introduz a seguinte modificação no termo de fixação de gauge em (A.48)

$$
-\frac{1}{\xi}\left(G^{\mu} A_{\mu}^{a}\right)(\delta \lambda)\left(G^{\nu} D_{\nu}^{a b} c_{2}^{b}\right)
$$

A invariância é recuperada, se definirmos a variação de $c_{1}$ em (A.45) como sendo dada por

$$
\delta c_{1}^{a}=i(\delta \lambda) \frac{1}{\xi} \partial^{\mu} A_{\mu}^{a}
$$

No entanto, a ação para os campos de fantasmas em (A.45) também vai se transformar sob (A.64) e (A.66). Esta transformação pode ser compensada de tal forma a determinar a correspondente variação do campo $c_{2}$. É possível mostrar, usando integração por partes e a identidade de Jacobi dada em (A.23), que a transformação procurada é

$$
\delta c_{2}^{a}=-\frac{1}{2}(\delta \lambda) f^{a b c} c_{2}^{b} c_{2}^{c}
$$

Observe que a anti-simetria de $f^{a b c}$ e o caráter grasmaniano dos campos $c$ fazem com que o lado direito da expressão acima seja não nulo.

Outra importante propriedade das transformações (A.64), (A.66) e (A.67) é que elas mantém invariante a medida de integração funcional em (A.47). Isto ocorre porque o 
determinante das matrizes (jacobiano)

$$
\begin{aligned}
\frac{\delta\left(A_{\mu}^{a}(x)+\delta A_{\mu}^{a}(x)\right)}{\delta A_{\nu}^{b}(y)} & =\delta^{4}(x-y) \delta_{\mu}^{\nu}\left(\delta^{a b}+(\delta \lambda) f^{a b c} c_{2}^{c}\right) \\
\frac{\delta\left(c_{1}^{a}(x)+\delta c_{1}^{a}(x)\right)}{\delta c_{1}^{b}(y)} & =\delta^{4}(x-y) \delta^{a b} \\
\frac{\delta\left(c_{2}^{a}(x)+\delta c_{2}^{a}(x)\right)}{\delta c_{2}^{b}(y)} & =\delta^{4}(x-y)\left(\delta^{a b}-(\delta \lambda) f^{a b c} c_{2}^{c}\right)
\end{aligned}
$$

é igual a um (isto pode ser facilmente verificado usando a propriedade grasmaniana $(\delta \lambda)^{2}=$ 0 e a anti-simetria de $\left.f^{a b c}\right)$.

Sumarizando, as transformações (A.64), (A.66) e (A.67) deixam a ação (A.49) invariante, generalizando assim a simetria de gauge da ação clássica. A descoberta desta simetria foi feita independentemente por mais de um grupo de pessoas, tendo sido denominadas transformações de BRST [32, 33, 34]. Ela desempenha um papel importante no estudo das propriedades de renormalizabilidade da teoria de gauge. De maneira geral esta simetria permite estabelecer relações entre diferentes amplitudes quânticas (ou térmicas) da teoria.

\section{A.2 Teoria de Yang-Mills em espaços não-comutativos}

O formalismo geral de teoria de campos em espaços não comutativos apresentado na seção (2.2) pode, em princípio, ser aplicado a qualquer teoria de campos. Na versão não comutativa da densidade de lagrangiana da teoria, cada um dos campos e suas derivadas são substituídos pelos correspondentes operadores de Weyl. A ação da teoria é então obtida pela operação de traço, introduzida em (2.12), e a relação (2.24) é empregada. Finalmente, a forma explícita do produto de Grönenwold-Moyal dado por (2.20) é empregado.

Poderíamos então supor que a versão não comutativa da teoria de Yang-Mills descrita pela lagrangiana (A.26) seguiria os passos descritos acima sem nenhuma condição adicional. Há no entanto um importante detalhe físico, específico das teorias de gauge, que deve ser levado em conta. Devemos lembrar que a construção da teoria de Yang-Mills usual poderia ter sido feita levando em conta não apenas a simetria sob transformações de $S(N)$, mas 
sim o grupo $U(N)=U(1) \times S U(N) / \mathbb{Z}_{N}$. Porém, na teoria de campos comutativa, o "fóton", associado à $U(1)$, se desacopla dos $N^{2}-1$ campos de gauge associados à $S U(N)$. O mesmo não vai ocorrer na versão não-comutativa da teoria de Yang-Mills. De fato, se $g$ e $h$ são dois elementos do grupo, em geral $\operatorname{det}(g \star h) \neq \operatorname{det}(g) \star \operatorname{det}(h)$. Portanto, o grupo especial unitário não é um grupo no espaço não-comutativo. Por outro lado, devido à propriedade $(g \star h)^{\dagger}=h^{\dagger} \star g^{\dagger}$, o produto $g \star h$ de duas matrizes unitárias é sempre unitário. A possibilidade de se introduzir outros grupos de gauge é restringida se quisermos manter a associatividade da álgebra não-comutativa [35, 36].

Somos então levados a considerar a seguinte versão não-comutativa da ação de YangMills

$$
S_{\mathrm{nc}}^{\mathrm{YM}}=\int d^{4} x \operatorname{Tr}\left(-\frac{1}{4} F_{\mu \nu} F^{\mu \nu}-\frac{1}{2 \alpha}\left(\partial_{\mu} A^{\mu}\right)^{2}+\frac{1}{2}\left(i c_{1} * \partial_{\mu} D^{\mu} c_{2}-i \partial_{\mu} D^{\mu} c_{2} * c_{1}\right)\right)
$$

onde

$$
F_{\mu \nu}=\partial_{\mu} A_{\nu}-\partial_{\nu} A_{\mu}-i g\left[A_{\mu}, A_{\nu}\right]_{\star}
$$

e

$$
A_{\mu}=A_{\mu}^{A} T^{A}, \quad A=0 \cdots N^{2}-1
$$

A versão não-comutativa da transformação de gauge em (A.15) é6 .

$$
A_{\mu} \rightarrow A_{\mu}+\partial_{\mu} \omega+i\left(\omega \star A_{\mu}-A_{\mu} \star \omega\right)
$$

ou, equivalentemente,

$$
A_{\mu}^{A} T^{A} \rightarrow A_{\mu}^{A} T^{A}+\partial_{\mu} \omega^{A} T^{A}+\frac{i}{2}\left[\omega^{A}, A_{\mu}^{B}\right]_{\star}\left\{T^{A}, T^{B}\right\}+\frac{i}{2}\left\{\omega^{A}, A_{\mu}^{B}\right\}_{\star}\left[T^{A}, T^{B}\right]
$$

Usando as relações,

$$
\left[T^{A}, T^{B}\right]=i f_{A B C} T^{C}, \quad\left\{T^{A}, T^{B}\right\}=d_{A B C} T^{C}, \quad \operatorname{Tr}\left(T^{A} T^{B}\right)=\delta^{A B},
$$

${ }^{6}$ Estas transformações são compatíveis com a condição de unitariedade

$$
U(x) \star U^{\dagger}(x)=U^{\dagger}(x) \star U(x)=I_{N} .
$$


onde as grandezas $f_{A B C}$ e $d_{A B C}$ são as constantes de estrutura do grupo $U(N)$ respectivamente simétrica e anti-simétrica, podemos escrever

$$
A_{\mu}^{A} \rightarrow A_{\mu}^{A}+\partial_{\mu} \omega^{A}+\frac{i}{2}\left[\omega^{A}, A_{\mu}^{B}\right]_{\star} d_{A B C}-\frac{1}{2}\left\{\omega^{A}, A_{\mu}^{B}\right\}_{\star} f_{A B C}
$$

Note que mesmo no caso $N=1$ (QED não comutativa), quando $T^{0}=1$ e $d_{000}=2$, as transformações mantém um caráter não-abeliano.

Usando identidades do tipo

$$
\left[A_{\mu}, A_{\nu}\right]_{\star}=\frac{1}{2}\left\{A_{\mu}^{A}, A_{\nu}^{B}\right\}_{\star}\left[T_{\mu}^{A}, T_{\nu}^{B}\right]+\frac{1}{2}\left[A_{\mu}^{A}, A_{\nu}^{B}\right]_{\star}\left\{T_{\mu}^{A}, T_{\nu}^{B}\right\}
$$

bem como as relações (A.75), a ação (A.69) pode ser expressa diretamente em termos dos campos $A_{\mu}^{A}, c_{1}^{A}$ e $c_{2}^{A}$. Em seguida, usando (2.21) e (2.24) e expressando os campos em termos de suas componentes de Fourier, as regras de Feynman podem ser lidas diretamente de $i S_{\mathrm{nc}}^{\mathrm{YM}}$. Os resultados assim obtidos generalizam as regras de Feynman da teoria usual, dadas em (A.59b), (A.59c) e (A.59d), pela substituição das constantes de estrutura fabc por

$$
C^{A B C}\left(p_{1}, p_{2}\right)=f^{A B C} \cos \left(\frac{p_{1} \times p_{2}}{2}\right)+d^{A B C} \sin \left(\frac{p_{1} \times p_{2}}{2}\right) ; \quad p_{1} \times p_{2} \equiv p_{1}^{\mu} \theta_{\mu \nu} p_{2}^{\nu}
$$

e levando em conta que $p_{1}, p_{2}$ e $p_{3}$ estão associados aos campos cuja cor é respectivamente $A, B$ e $C$. Os propagadores são obtidos de (A.55) e (A.57) fazendo $\delta^{a b} \rightarrow \delta^{A B}$. A teoria perturbativa assim definida possui as propriedades de invariância sob transformações de BRST e é renormalizável [37]. 


\section{Apêndice B}

\section{Cálculo das constates do tensor $C^{(n)}$}

Neste apêndice mostraremos como calculamos os valores das constantes do tensor de Lorentz $C^{(2)}$ e faremos uma breve introdução ao pacote de cálculo algébrico que contem todos os comandos de manipulação tensorial que foram usados no Maple V.

Iniciamos nossos cálculos iniciando os pacotes de análise combinatória (combinat) e de cálculo tensorial (hip)[38],

$>$ restart;with(combinat):

$>\operatorname{read}(\mathrm{hip})$

Para realizamos as contrações para a determinação das constantes é necessário definirmos os índices e os vetores. As seguintes grandezas serão utilizadas como índices mudos,

$>\operatorname{setindex}(\mathrm{m} 1, \mathrm{n} 1, \mathrm{~m} 2, \mathrm{n} 2, \mathrm{~m} 3, \mathrm{n} 3)$;

e estas serão utilizadas como vetores $(\mathrm{k} 1, \mathrm{k} 2, \mathrm{k} 3, \mathrm{k} 4)$ ou $(\mathrm{x}, \mathrm{y}, \mathrm{x} 1, \mathrm{y} 1, \mathrm{x} 2$, y2 , x3 , y3) como índices não contraídos.

$>\operatorname{setfv}(\mathrm{x}, \mathrm{y}, \mathrm{x} 1, \mathrm{y} 1, \mathrm{x} 2, \mathrm{y} 2, \mathrm{x} 3, \mathrm{y} 3, \mathrm{k} 1, \mathrm{k} 2, \mathrm{k} 3, \mathrm{k} 4)$; 
Assim, por exemplo, temos a seguinte correspondência com a notação usual,

$$
\begin{aligned}
\mathrm{x} & \rightarrow \mu \\
\mathrm{x} 1 & \rightarrow \mu_{1} \\
\mathrm{x} 2 & \rightarrow \mu_{2} \\
\mathrm{y} & \rightarrow \nu \\
\mathrm{y} 1 & \rightarrow \nu_{1} \\
\mathrm{y} 2 & \rightarrow \nu_{1}
\end{aligned}
$$

\section{B.1 Cálculo de $C^{(1)}$}

Agora que definimos nossas variáveis, vamos calcular o termo de ordem mais baixa e determinar as constantes de um tersor qualquer possuindo 4 índices,

$>\operatorname{perm0}:=\operatorname{permute}([\mathrm{x} 1, \mathrm{y} 1, \mathrm{x} 2, \mathrm{y} 2])$;

$$
\begin{aligned}
& \text { perm0 := [[x1,y1,x2,y2], [x1,y1,y2,x2], [x1,x2,y1,y2], } \\
& {[x 1, x 2, y 2, y 1],[x 1, y 2, y 1, x 2],[x 1, y 2, x 2, y 1] \text {, }} \\
& {[y 1, x 1, x 2, y 2],[y 1, x 1, y 2, x 2],[y 1, x 2, x 1, y 2] \text {, }} \\
& {[y 1, x 2, y 2, x 1],[y 1, y 2, x 1, x 2],\left[y 1, y^{2}, x 2, x 1\right] \text {, }} \\
& {[x 2, x 1, y 1, y 2],[x 2, x 1, y 2, y 1],[x 2, y 1, x 1, y 2] \text {, }} \\
& {[x 2, y 1, y 2, x 1],[x 2, y 2, x 1, y 1],[x 2, y 2, y 1, x 1] \text {, }} \\
& {[y 2, x 1, y 1, x 2],[y 2, x 1, x 2, y 1],[y 2, y 1, x 1, x 2] \text {, }} \\
& [y 2, y 1, x 2, x 1],[y 2, x 2, x 1, y 1],[y 2, x 2, y 1, x 1]]
\end{aligned}
$$

para calcularmos o tensor com 4 índices precisamos determinar a estrutura tensorial básica envolvendo a métrica de Minkowski, dada por,

$>$ unapply $(\mathrm{x} 1 \& \cdot \mathrm{y} 1 * \mathrm{x} 2 \& \cdot \mathrm{y} 2, \mathrm{x} 1, \mathrm{y} 1, \mathrm{x} 2, \mathrm{y} 2)$;

$$
(x 1, y 1, x 2, y 2) \rightarrow(x 1 \& \cdot y 1)\left(x_{2} \& . y 2\right)
$$

A notação adotada para o produto escalar quando usamos o pacote HIP é representada pelo símbolo \&.. Agora, substituindo cada elemento da lista perm0 na estrutura acima e 
eliminando a repetição de tensores idênticos, temos assim, a seguinte base tensorial

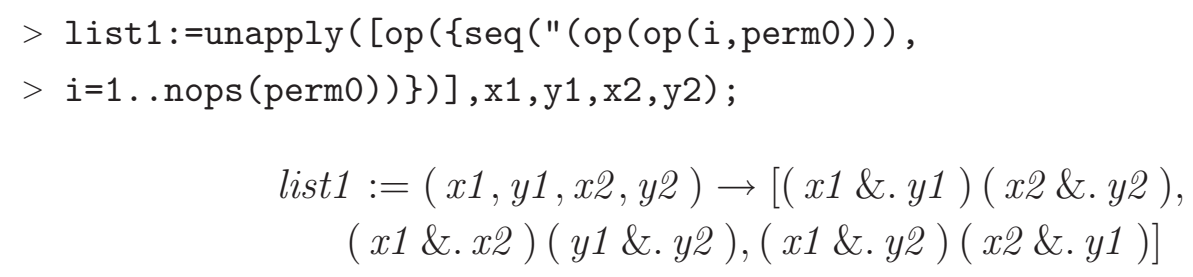

Estes três tensores devem ser agrupados de tal forma que o conjunto de tensores em cada grupo possua a simetria pela troca de pares de índices correspondentes ao campo gravitacional. "Escolhendo os índices x1 e y1 como sendo os índices a serem contraídos com o tensor de campo gravitacional, vemos que o primeiro tensor de list1 é simétrico enquanto a soma dos dois últimos também forma um tensor simétrico. Há portantoduas combinações possíveis, ou seja, teremos duas constantes a serem determinadas". Este procedimento foi feito no capítulo 3 quando calculamos os valores das constantes $a$ e $b$.

\section{B.2 Cálculo de $C^{(2)}$}

Consideremos um tensor qualquer possuindo seis índices. Com esta configuração de índices ao permutarmos, teremos um total de 720 tensores distintos. Como mostra o cálculo abaixo,

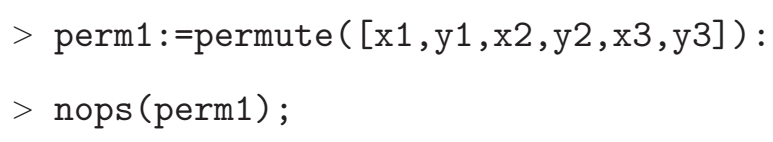

O tipo de estrutura tensorial que nos interessa é aquela em que tenhamos produtos de métricas de Minkowski. Que será dada por:

$>$ unapply $(\mathrm{x} 1 \& \cdot \mathrm{y} 1 * \mathrm{x} 2 \& \cdot \mathrm{y} 2 * \mathrm{x} 3 \& \cdot \mathrm{y} 3, \mathrm{x} 1, \mathrm{y} 1, \mathrm{x} 2, \mathrm{y} 2, \mathrm{x} 3, \mathrm{y} 3)$;

$$
(x 1, y 1, x 2, y 2, x 3, y 3) \rightarrow(x 1 \& . y 1)\left(x_{2} \& . y 2\right)(x 3 \& . y 3)
$$


Quando temos essa estrutura temos que os 720 tensores se reduzem a 15 tensores

$>$ list1: $=\operatorname{unapply}([\mathrm{op}(\{\operatorname{seq}("(\operatorname{op}(\mathrm{op}(i, \mathrm{perm} 1)))$,

$>\mathrm{i}=1 \ldots \operatorname{nops}($ perm1) ) $\})], \mathrm{x} 1, \mathrm{y} 1, \mathrm{x} 2, \mathrm{y} 2, \mathrm{x} 3, \mathrm{y} 3)$;

list1 $:=(x 1, y 1, x 2, y 2, x 3, y 3) \rightarrow[(x 1 \& . y 1)(x 2 \& . y 2)(x 3 \& . y 3)$,

( $x 1 \& . y 1)(x 2 \& . x 3)$ ( y2 \&.y3), ( $x 1$ \&. x2) (y1 \&. y3) (x3 \&. y2),

( 11 \&.y2) (x2 \&.y1) (x3 \&.y3), (x1 \&. y2) (x3 \&. y1) (x2 \&. y3),

$(x 1 \& . y 2)(y 1 \& . y 3)(x 2 \& . x 3),(x 1 \& . x 3)(x 2 \& . y 1)($ y2 \&.y3),

( $x 1$ \&.x3) (y1 \&.y3) (x2 \&. y2), ( 11 \&. y1) (x2 \&. y3) (x3 \&. y2),

( $x 1 \& . x 2)(y 1 \& . y 2)(x 3 \& . y 3),(x 1 \& . y 3)(x 2 \& . y 1)(x 3 \& . y 2)$,

(

( $x 1$ \&.y3) (y1 \&.y2) (x2 \&.x3), (x1 \&.y3) (x3\&.y1) (x2 \&. y2)]

$>\operatorname{nops}(\operatorname{list1}(\mathrm{x} 1, \mathrm{y} 1, \mathrm{x} 2, \mathrm{y} 2, \mathrm{x} 3, \mathrm{y} 3))$;

Vamos agora agrupar os 15 tensores de tal forma que o conjunto de tensores em cada grupo possua simetria pela troca de pares de de índices correspondentes ao campo gravitacional.

$>$ bas1:=unapply $(\mathrm{x} \& \cdot \mathrm{y} * \mathrm{x} 1 \& \cdot \mathrm{y} 1 * \mathrm{x} 2 \& \cdot \mathrm{y} 2, \mathrm{x}, \mathrm{y}, \mathrm{x} 1, \mathrm{y} 1, \mathrm{x} 2, \mathrm{y} 2)$;

$$
\text { bas1 }:=(x, y, x 1, y 1, x 2, y 2) \rightarrow(x \& . y)(x 1 \& . y 1)(x 2 \& . y 2)
$$

Para isso vamos considerar os seguintes seis tensores:

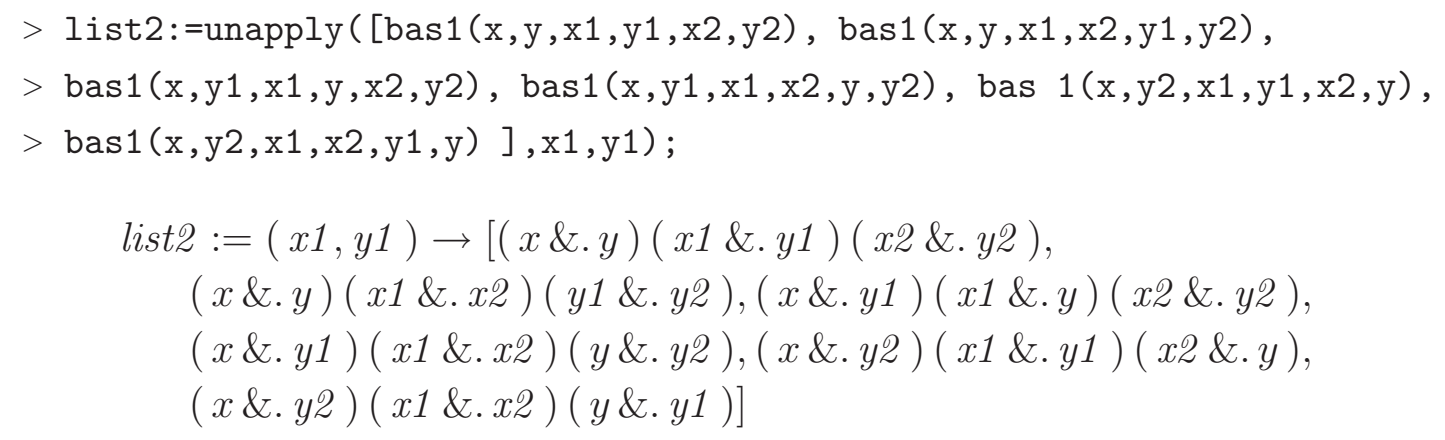

Simetrizando nos índices correspondentes aos campos gravitacionais, temos,

$>\operatorname{unapply}([\mathrm{op}(\{\mathrm{op}("(\mathrm{x} 1, \mathrm{y} 1))\} \operatorname{union}\{\mathrm{op}("(\mathrm{y} 1, \mathrm{x} 1))\})], \mathrm{x} 2, \mathrm{y} 2)$; 


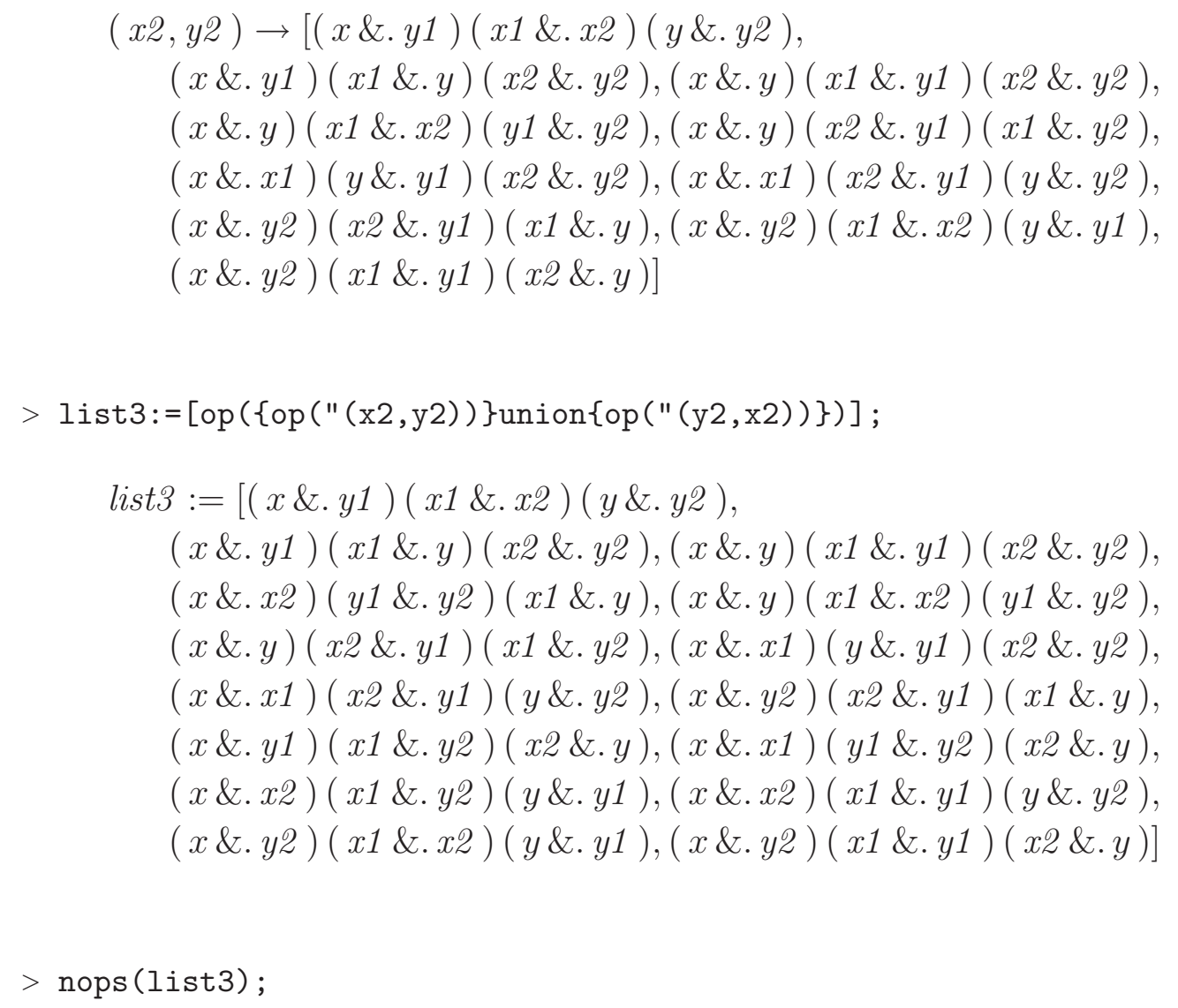

$>\{o p($ list 3$)\} \operatorname{minus}\{o p(\operatorname{list1}(\mathrm{x}, \mathrm{y}, \mathrm{x} 1, \mathrm{y} 1, \mathrm{x} 2, \mathrm{y} 2))\}$

Após simetrizarmos, os índices introduzimos as constantes que queremos determinar, escrevendo o seguinte comando,

$>[\operatorname{seq}(c . i * o p(i, \operatorname{list} 2(x 1, y 1)), i=1.6)] ;$

[c1 (x\&.y) (x1 \&.y1) (x2 \&.y2), c2 ( $x \& . y)(x 1 \& . x 2)(y 1 \& . y 2)$,

c3 $(x \& . y 1)(x 1 \& . y)(x 2 \& . y 2), c 4(x \& . y 1)(x 1 \& . x 2)(y \& . y 2)$,

$c 5$ ( $x \& . y 2)(x 1 \& . y 1)(x 2 \& . y), c 6(x \& . y 2)(x 1 \& . x 2)(y \& . y 1)]$

Agora que temos as constantes vamos para achá-las precisamos escrever a combinação linear entre elas como segue,

$>\operatorname{unapply}(\operatorname{sum}(o p(j j, "), j j=1 \ldots 6), x 1, y 1)$; 


$$
\begin{aligned}
& (x 1, y 1) \rightarrow c 1(x \& . y)(x 1 \& . y 1)(x 2 \& . y 2) \\
& +c 2(x \& . y)(x 1 \& . x 2)(y 1 \& . y 2)+c 3(x \& . y 1)(x 1 \& . y)(x 2 \& . y 2) \\
& +c 4(x \& . y 1)(x 1 \& . x 2)(y \& . y 2)+c 5(x \& . y 2)(x 1 \& . y 1)(x 2 \& . y) \\
& +c 6\left(x \& . y^{2}\right)(x 1 \& . x 2)(y \& . y 1) \\
& >\text { unapply(" }(\mathrm{x} 1, \mathrm{y} 1) / 2+"(\mathrm{y} 1, \mathrm{x} 1) / 2, \mathrm{x} 2, \mathrm{y} 2) \text {; } \\
& \left(x 2, y^{2}\right) \rightarrow c 1(x \& . y)(x 1 \& . y 1)\left(x^{2} \& . y^{2}\right) \\
& +\frac{1}{2} c_{2}(x \& \cdot y)(x 1 \& \cdot x 2)(y 1 \& \cdot y 2)+\frac{1}{2} c 3(x \& \cdot y 1)(x 1 \& \cdot y)(x 2 \& . y 2) \\
& +\frac{1}{2} c_{4}(x \& \cdot y 1)(x 1 \& . x 2)(y \& . y 2)+c 5(x \& . y 2)(x 1 \& . y 1)(x 2 \& \cdot y) \\
& +\frac{1}{2} c 6\left(x \& . y^{2}\right)\left(x 1 \& . x_{2}\right)(y \& \cdot y 1)+\frac{1}{2} c_{2}(x \& . y)(x 2 \& \cdot y 1)(x 1 \& . y 2) \\
& +\frac{1}{2} c_{3}(x \& \cdot x 1)(y \& \cdot y 1)(x 2 \& \cdot y 2)+\frac{1}{2} c_{4}(x \& \cdot x 1)(x 2 \& \cdot y 1)(y \& \cdot y 2) \\
& +\frac{1}{2} c 6(x \& \cdot y 2)(x 2 \& \cdot y 1)(x 1 \& \cdot y)
\end{aligned}
$$

$$
\begin{aligned}
& C 2 a:=(x, y, x 1, y 1, x 2, y 2) \rightarrow c 1(x \& . y)(x 1 \& . y 1)(x 2 \& . y 2) \\
& +\frac{1}{2} c_{2}(x \& \cdot y)\left(x 1 \& \cdot x^{2}\right)\left(y 1 \& \cdot y^{2}\right)+\frac{1}{2} c_{3}(x \& \cdot y 1)(x 1 \& \cdot y)\left(x \mathscr{2} \& \cdot y^{2}\right) \\
& +\frac{1}{4} c_{4}(x \& . y 1)(x 1 \& . x 2)(y \& . y 2)+\frac{1}{2} c 5(x \& . y 2)(x 1 \& . y 1)(x 2 \& . y) \\
& +\frac{1}{4} c 6(x \& . y 2)(x 1 \& . x 2)(y \& \cdot y 1)+\frac{1}{2} c 2(x \& . y)(x \mathscr{2} \& \cdot y 1)(x 1 \& . y 2) \\
& +\frac{1}{2} c 3(x \& \cdot x 1)(y \& \cdot y 1)\left(x_{2} \& \cdot y^{2}\right)+\frac{1}{4} c_{4}(x \& \cdot x 1)(x 2 \& \cdot y 1)\left(y \& . y^{2}\right) \\
& +\frac{1}{4} c 6(x \& . y 2)(x 2 \& . y 1)(x 1 \& . y)+\frac{1}{4} c_{4}(x \& . y 1)(x 1 \& . y 2)(x 2 \& . y) \\
& +\frac{1}{2} c 5(x \& . x 2)(x 1 \& . y 1)(y \& \cdot y 2)+\frac{1}{4} c 6(x \& . x 2)(x 1 \& \cdot y 2)(y \& . y 1) \\
& +\frac{1}{4} c 4(x \& \cdot x 1)(y 1 \& . y 2)(x 2 \& . y)+\frac{1}{4} c 6(x \& . x 2)(y 1 \& . y 2)(x 1 \& \cdot y) \\
& >\operatorname{collect}(\mathrm{C} 2 \mathrm{a}(\mathrm{x}, \mathrm{y}, \mathrm{x} 1, \mathrm{y} 1, \mathrm{x} 2, \mathrm{y} 2),\{\mathrm{c} 1, \mathrm{c} 2, \mathrm{c} 3, \mathrm{c} 4, \mathrm{c} 5, \mathrm{c} 6\}) \text {; }
\end{aligned}
$$




$$
\begin{aligned}
& c 1(x \& . y)(x 1 \& . y 1)(x 2 \& . y 2) \\
& +\left(\frac{1}{2}(x \& \cdot y)(x 2 \& \cdot y 1)(x 1 \& \cdot y 2)++\frac{1}{2}(x \& \cdot y)(x 1 \& \cdot x 2)(y 1 \& \cdot y 2)\right) c^{2} \\
& +\left(\frac{1}{2}(x \& \cdot y 1)(x 1 \& \cdot y)(x 2 \& \cdot y 2)+\frac{1}{2}(x \& \cdot x 1)(y \& \cdot y 1)(x 2 \& \cdot y 2)\right) c 3 \\
& +\left(\frac{1}{4}(x \& . x 1)(y 1 \& . y 2)(x 2 \& . y)+\frac{1}{4}(x \& . y 1)(x 1 \& . y 2)(x 2 \& . y)\right. \\
& \left.+\frac{1}{4}(x \& \cdot y 1)(x 1 \& \cdot x \mathscr{2})(y \& \cdot y 2)+\frac{1}{4}(x \& \cdot x 1)(x 2 \& \cdot y 1)(y \& \cdot y 2)\right) c_{4} \\
& +\left(\frac{1}{2}\left(x \& \cdot x^{2}\right)(x 1 \& \cdot y 1)\left(y \& . y^{2}\right)+\frac{1}{2}\left(x \& . y^{2}\right)(x 1 \& . y 1)(x \mathscr{2} \& \cdot y)\right) c 5 \\
& +\left(\frac{1}{4}(x \& \cdot y 2)(x 2 \& \cdot y 1)(x 1 \& \cdot y)+\frac{1}{4}(x \& \cdot y 2)(x 1 \& \cdot x 2)(y \& \cdot y 1)\right. \\
& \left.+\frac{1}{4}(x \& \cdot x \mathscr{2})\left(x 1 \& \cdot y^{2}\right)(y \& \cdot y 1)+\frac{1}{4}(x \& \cdot x \mathscr{2})(y 1 \& \cdot y 2)(x 1 \& \cdot y)\right) c 6 \\
& >\mathrm{C} 2:=\text { unapply (collect ( } \operatorname{subs}(\{\mathrm{c} 1=\mathrm{a} 1, \mathrm{c} 2=\mathrm{a} 2, \mathrm{c} 3=\mathrm{a} 3, \mathrm{c} 4=\mathrm{a} 4, \mathrm{c} 5=\mathrm{a} 5, \mathrm{c} 6=\mathrm{a} 6\}, ") \text {, } \\
& >\{a 1, a 2, a 3, a 4, a 5, a 6\}), x, y, x 1, y 1, x 2, y 2) \text {; } \\
& \text { C2 }:=(x, y, x 1, y 1, x 2, y 2) \rightarrow a 1(x \& . y)(x 1 \& . y 1)(x 2 \& . y 2) \\
& +\left(\frac{1}{2}(x \& \cdot y)\left(x 1 \& \cdot y^{2}\right)(x \mathscr{2} \& \cdot y 1)+\frac{1}{2}(x \& \cdot y)(x 1 \& \cdot x \mathscr{2})(y 1 \& \cdot y 2)\right) a^{2} \\
& +\left(\frac{1}{2}(x \& \cdot y 1)(x 1 \& \cdot y)(x 2 \& \cdot y 2)\right. \\
& \left.+\frac{1}{2}(x \& \cdot x 1)(y \& \cdot y 1)(x 2 \& \cdot y 2)\right) \text { a3 } \\
& +\left(\frac{1}{4}(x \& \cdot x 1)(x 2 \& \cdot y)(y 1 \& \cdot y 2)+\frac{1}{4}(x \& \cdot y 1)(x 2 \& \cdot y)(x 1 \& \cdot y 2)\right. \\
& \left.+\frac{1}{4}(x \& \cdot y 1)(y \& \cdot y 2)(x 1 \& \cdot x 2)+\frac{1}{4}(x \& \cdot x 1)(y \& \cdot y 2)(x 2 \& \cdot y 1)\right) a_{4} \\
& +\left(\frac{1}{2}(x \& \cdot x 2)(y \& \cdot y 2)(x 1 \& \cdot y 1)+\frac{1}{2}(x \& . y 2)(x 2 \& \cdot y)(x 1 \& \cdot y 1)\right) a 5 \\
& +\left(\frac{1}{4}(x \& \cdot y 2)(x 1 \& \cdot y)(x 2 \& \cdot y 1)+\frac{1}{4}(x \& \cdot y 2)(y \& \cdot y 1)(x 1 \& \cdot x 2)\right. \\
& \left.+\frac{1}{4}(x \& \cdot x \mathscr{2})(y \& \cdot y 1)(x 1 \& \cdot y \mathscr{2})+\frac{1}{4}(x \& \cdot x \mathscr{2})(x 1 \& \cdot y)(y 1 \& \cdot y 2)\right) a 6
\end{aligned}
$$

Vamos agora encontrar os coeficientes dos dois anti-comutadores. O que queremos 
realmente é encontrar os valores das constantes do tensor de Lorentz. Usando as condições de simetria dos campos temos uma redução no número de nossas constantes. Como mostra o cálculo abaixo esta redução se da pelo fato de que alguns termos são iguais pela troca de índices levando assim numa interpretação de igualdade entre eles com mostraremos agora.

$$
\begin{aligned}
& >\text { Css: }=\text { unapply }(\operatorname{collect}(\operatorname{subs}(\{\mathrm{a} 5=\mathrm{a} 3, \mathrm{a} 6=\mathrm{a} 4\}, \mathrm{C} 2(\mathrm{x}, \mathrm{y}, \mathrm{x} 1, \mathrm{y} 1, \mathrm{x} 2, \mathrm{y} 2)), \\
& >\{\mathrm{a} 1, \mathrm{a} 2, \mathrm{a} 3, \mathrm{a} 4\}), \mathrm{x}, \mathrm{y}, \mathrm{x} 1, \mathrm{y} 1, \mathrm{x} 2, \mathrm{y} 2)
\end{aligned}
$$

$$
\begin{aligned}
& \text { Css }:=(x, y, x 1, y 1, x 2, y 2) \rightarrow \\
& \left(\frac{1}{2}(x \& . y 1)(x 1 \& \cdot y)(x \mathscr{2} \& \cdot y 2)+\frac{1}{2}(x \& . x 1)(y \& \cdot y 1)(x \mathscr{2} \& \cdot y 2)\right. \\
& \left.+\frac{1}{2}\left(x \& \cdot x^{2}\right)\left(y \& \cdot y^{2}\right)(x 1 \& \cdot y 1)+\frac{1}{2}\left(x \& \cdot y^{2}\right)(x 2 \& \cdot y)(x 1 \& \cdot y 1)\right) a_{3} \\
& +\left(\frac{1}{4}(x \& \cdot x 1)(x 2 \& \cdot y)(y 1 \& \cdot y 2)+\frac{1}{4}(x \& \cdot y 1)(x 2 \& \cdot y)(x 1 \& \cdot y 2)\right. \\
& +\frac{1}{4}(x \& \cdot y 1)\left(y \& \cdot y^{2}\right)\left(x 1 \& \cdot x^{2}\right)+\frac{1}{4}(x \& . x 1)\left(y \& \cdot y^{2}\right)(x 2 \& \cdot y 1) \\
& +\frac{1}{4}(x \& \cdot y 2)(x 1 \& \cdot y)(x 2 \& \cdot y 1)+\frac{1}{4}(x \& . y 2)(y \& \cdot y 1)(x 1 \& . x 2) \\
& \left.+\frac{1}{4}(x \& \cdot x 2)(y \& \cdot y 1)\left(x 1 \& \cdot y^{2}\right)+\frac{1}{4}(x \& \cdot x 2)(x 1 \& \cdot y)(y 1 \& \cdot y 2)\right) a_{4} \\
& +a 1(x \& . y)(x 1 \& . y 1)(x 2 \& . y 2) \\
& +\left(\frac{1}{2}(x \& \cdot y)(x 1 \& \cdot y 2)(x \mathscr{2} \& \cdot y 1)+\frac{1}{2}(x \& \cdot y)(x 1 \& \cdot x 2)(y 1 \& \cdot y 2)\right) a^{2}
\end{aligned}
$$

Neste ponto, vamos checar se esse tensor é simétrico pela troca de x1 por x2 e y1 por y2, fazemos isso da seguinte maneira,

$$
>\operatorname{Css}(\mathrm{x}, \mathrm{y}, \mathrm{x} 1, \mathrm{y} 1, \mathrm{x} 2, \mathrm{y} 2)-\operatorname{Css}(\mathrm{x}, \mathrm{y}, \mathrm{x} 2, \mathrm{y} 2, \mathrm{x} 1, \mathrm{y} 1) \text {; }
$$

$>\operatorname{Css}(x, y, x 1, y 2, x 2, y 1)-\operatorname{Css}(x, y, x 2, y 1, x 1, y 2)$;

$>\operatorname{Css}(\mathrm{x}, \mathrm{y}, \mathrm{y} 1, \mathrm{y} 2, \mathrm{x} 2, \mathrm{x} 1)-\operatorname{Css}(\mathrm{x}, \mathrm{y}, \mathrm{x} 2, \mathrm{x} 1, \mathrm{y} 1, \mathrm{y} 2)$ 
Vimos anteriormente que a variação de primeira ordem da ação envolve uma quantidade que possui o produto de dois tensores de Minkowisk, sendo esta quantidade expressa pela seguinte forma.

$>\mathrm{C} 1:=$ unapply $(\mathrm{x} 1 \& \cdot \mathrm{y} 1 * \mathrm{x} 2 \& \cdot \mathrm{y} 2-\mathrm{x} 1 \& \cdot \mathrm{x} 2 * \mathrm{y} 1 \& \cdot \mathrm{y} 2-\mathrm{x} 1 \& \cdot \mathrm{y} 2 * \mathrm{y} 1 \& \cdot \mathrm{x} 2, \mathrm{x} 1, \mathrm{y} 1, \mathrm{x} 2, \mathrm{y} 2) ;$

$$
\begin{aligned}
C 1 & :=(x 1, y 1, x 2, y 2) \rightarrow(x 1 \& \cdot y 1)(x 2 \& . y 2)-(x 1 \& . x 2)(y 1 \& . y 2) \\
& -\left(x 1 \& . y_{2}\right)(x 2 \& . y 1)
\end{aligned}
$$

Com essa estrutura podemos determinar a variação do termo de primeira ordem levando em conta a substituição da transformada de Fourier dos campos, como segue,

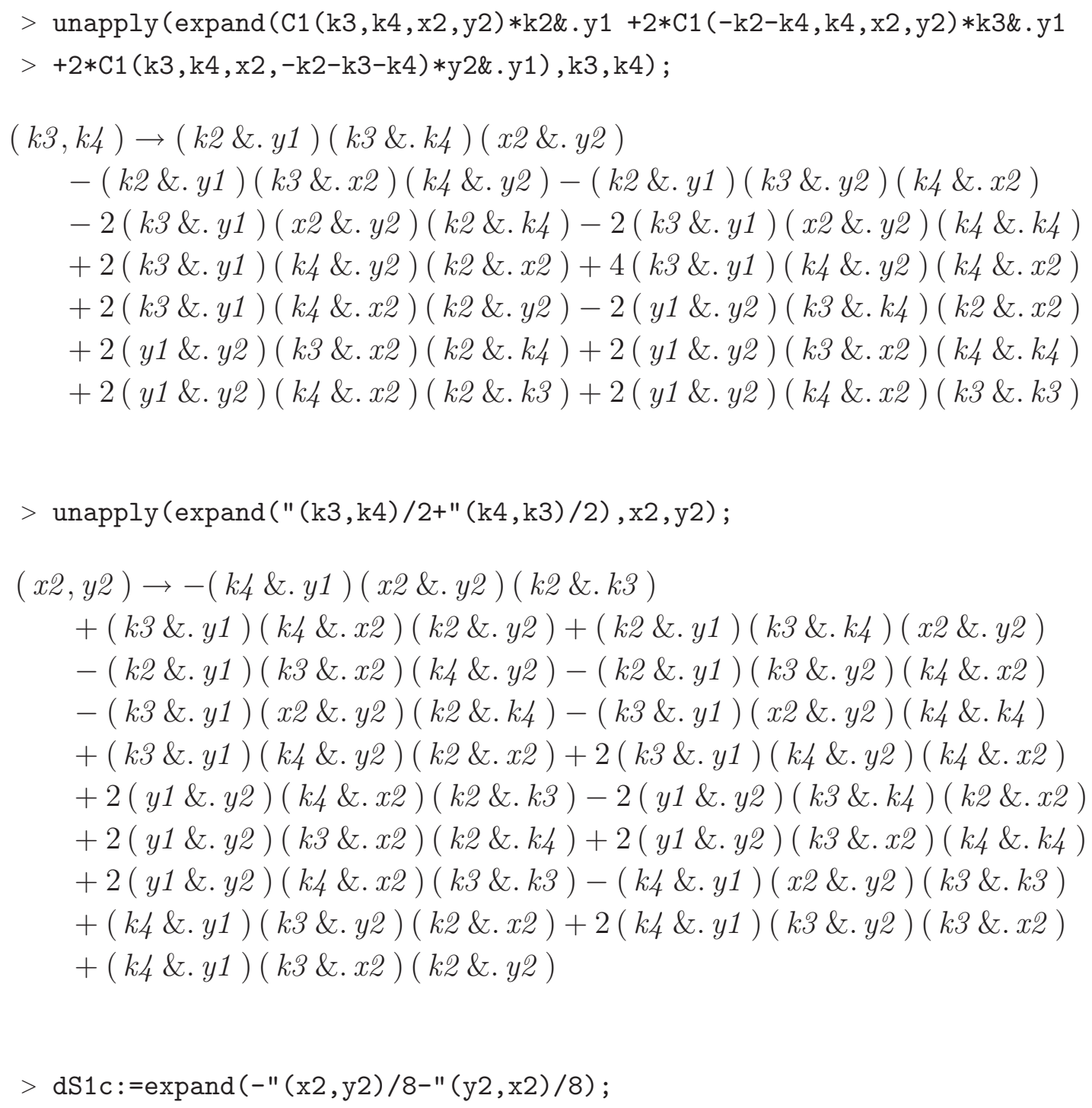




$$
\begin{aligned}
& d S 1 c:=\frac{1}{4}(k 4 \& \cdot y 1)(x 2 \& \cdot y 2)(k 2 \& \cdot k 3)-\frac{1}{4}(k 3 \& \cdot y 1)(k 4 \& \cdot x 2)(k 2 \& \cdot y 2) \\
& -\frac{1}{4}(k 2 \& \cdot y 1)\left(k 3 \& \cdot k_{4}\right)\left(x_{2} \& \cdot y_{2}\right)+\frac{1}{4}(k 2 \& \cdot y 1)\left(k 3 \& \cdot x_{2}\right)\left(k_{4} \& \cdot y_{2}\right) \\
& +\frac{1}{4}(k 2 \& \cdot y 1)(k 3 \& \cdot y 2)\left(k_{4} \& \cdot x_{2}\right)+\frac{1}{4}\left(k_{3} \& \cdot y 1\right)\left(x_{2} \& \cdot y 2\right)\left(k 2 \& \cdot k_{4}\right) \\
& +\frac{1}{4}\left(k_{3} \& . y 1\right)\left(x_{2} \& \cdot y_{2}\right)\left(k_{4} \& . k_{4}\right)-\frac{1}{4}\left(k_{3} \& . y 1\right)\left(k_{4} \& . y 2\right)\left(k 2 \& . x_{2}\right) \\
& -\frac{1}{2}\left(k_{3} \& . y 1\right)\left(k_{4} \& . y 2\right)\left(k_{4} \& . x_{2}\right)-\frac{1}{4}(y 1 \& . y 2)\left(k_{4} \& . x_{2}\right)\left(k_{2} \& . k_{3}\right) \\
& +\frac{1}{4}(y 1 \& . y 2)\left(k 3 \& . k_{4}\right)\left(k 2 \& . x_{2}\right)-\frac{1}{4}(y 1 \& . y 2)\left(k 3 \& . x_{2}\right)\left(k 2 \& . k_{4}\right) \\
& -\frac{1}{4}(y 1 \& . y 2)\left(k 3 \& . x_{2}\right)\left(k_{4} \& . k_{4}\right)-\frac{1}{4}\left(y 1 \& . y_{2}\right)\left(k_{4} \& . x_{2}\right)\left(k_{3} \& . k_{3}\right) \\
& +\frac{1}{4}\left(k_{4} \& \cdot y 1\right)\left(x_{2} \& \cdot y_{2}\right)\left(k_{3} \& . k_{3}\right)-\frac{1}{4}\left(k_{4} \& . y 1\right)\left(k_{3} \& . y 2\right)\left(k 2 \& . x_{2}\right) \\
& -\frac{1}{2}\left(k_{4} \& \cdot y 1\right)\left(k_{3} \& \cdot y 2\right)\left(k_{3} \& . x_{2}\right)-\frac{1}{4}\left(k_{4} \& . y 1\right)\left(k_{3} \& . x_{2}\right)(k 2 \& . y 2) \\
& -\frac{1}{4}\left(x_{2} \& \cdot y 1\right)\left(k_{4} \& \cdot y_{2}\right)\left(k_{2} \& . k_{3}\right)+\frac{1}{4}\left(x_{2} \& . y 1\right)\left(k_{3} \& . k_{4}\right)\left(k_{2} \& \cdot y 2\right) \\
& -\frac{1}{4}\left(x_{2} \& . y 1\right)(k 3 \& . y 2)\left(k 2 \& . k_{4}\right)-\frac{1}{4}\left(x_{2} \& . y 1\right)(k 3 \& . y 2)\left(k_{4} \& . k_{4}\right) \\
& -\frac{1}{4}(x 2 \& \cdot y 1)(k 4 \& . y 2)(k 3 \& . k 3) \\
& >\operatorname{nops}(\mathrm{dS} 1 \mathrm{c}) \text {; }
\end{aligned}
$$

Agora vamos determinar a variação de segunda ordem tomando o tensor calculado anteriormente e denominado de Css:

> unapply (expand $(4 * \operatorname{Css}(\mathrm{k} 3, \mathrm{k} 4,-\mathrm{k} 2-\mathrm{k} 3-\mathrm{k} 4, \mathrm{y} 1, \mathrm{x} 2, \mathrm{y} 2)), \mathrm{k} 3, \mathrm{k} 4)$;

$$
\begin{aligned}
& \left(k_{3}, k_{4}\right) \rightarrow \\
& -2 \text { a3 (k3\&.y1) (x2 \&.y2) ( k3 \&. k4 ) - } 2 \text { a3 (k3\&.y1) ( x2 \&.y2) (k4 \&.k4) } \\
& -2 \text { a3 (k4\&.y1) (x2 \&.y2) ( k2 \&. k3) - } 2 \text { a3 ( k4 \&.y1) ( x2 \&.y2) ( k3 \&.k3) } \\
& -2 \text { a3 (k4\&.y1) (x2 \&.y2) (k3\&. k4 ) - } 2 \text { a3 (k2 \&.y1) (k3\&.x2) (k4\&.y2) } \\
& -2 \text { a3 (k3\&.x2) (k4\&.y2) (k3\&.y1)-2 a3 (k3\&.x2) (k4\&.y2) (k4\&.y1) } \\
& -2 \text { a3 (k2 \&.y1) (k3\&.y2) (k4\&.x2) - } 2 \text { a3 (k3\&.y2) (k4\&.x2) (k3 \&.y1) } \\
& -2 \text { a3 (k3\&.y2) (k4\&.x2) (k4 \&.y1)-a4 (y1 \&.y2) (k4\&.x2) (k2 \&.k3) } \\
& -a_{4}\left(y 1 \& . y_{2}\right)\left(k_{4} \& . x_{2}\right)\left(k_{3} \& . k_{3}\right)-a_{4}\left(k_{4} \& . x_{2}\right)\left(y 1 \& . y_{2}\right)\left(k_{3} \& . k_{4}\right)
\end{aligned}
$$


$-a_{4}(k 3 \& . y 1)\left(k_{4} \& . x 2\right)(k 2 \& . y 2)-a_{4}(k 3 \& . y 2)(k 4 \& . x 2)(k 3 \& . y 1)$

$-2 a_{4}(k 3 \& . y 1)\left(k_{4} \& . y 2\right)\left(k_{4} \& . x_{2}\right)-a_{4}\left(k_{3} \& . y 1\right)\left(k_{4} \& . y 2\right)$ (k2 \&. x2)

$-a_{4}\left(k 3 \& . x_{2}\right)\left(k_{4} \& . y 2\right)(k 3 \& . y 1)-a_{4}\left(x_{2} \& . y 1\right)\left(k_{4} \& . y 2\right)\left(k 2 \& . k_{3}\right)$

$-a_{4}\left(x_{2} \& . y 1\right)\left(k_{4} \& . y 2\right)\left(k 3 \& . k_{3}\right)-a_{4}\left(k_{4} \& . y 2\right)\left(x_{2} \& . y 1\right)\left(k 3 \& . k_{4}\right)$

$-a_{4}\left(x_{2} \& . y 1\right)(k 3 \& . y 2)\left(k 2 \& . k_{4}\right)-a 4(k 3 \& . y 2)(x 2 \& . y 1)(k 3 \& . k 4)$

$-a_{4}\left(x_{2} \& . y 1\right)(k 3 \& . y 2)\left(k_{4} \& . k_{4}\right)-a_{4}\left(k_{4} \& . y 1\right)\left(k_{3} \& . y 2\right)\left(k 2 \& . x_{2}\right)$

$-2 a_{4}\left(k_{4} \& . y 1\right)(k 3 \& . y 2)(k 3 \& . x 2)-a_{4}(k 3 \& . y 2)(k 4 \& . x 2)(k 4 \& . y 1)$

- $a_{4}\left(k_{4} \& . y 1\right)(k 3 \& . x 2)\left(k 2 \& . y_{2}\right)-a_{4}\left(k_{3} \& . x_{2}\right)\left(k_{4} \& \cdot y_{2}\right)\left(k_{4} \& \cdot y 1\right)$

$-a_{4}(y 1$ \&.y2) (k3\&.x2) (k2 \&. k4 ) - a4 (k3\&.x2) (y1 \&.y2) (k3\&.k4)

$-a_{4}\left(y 1 \& . y_{2}\right)(k 3 \& . x 2)\left(k_{4} \& . k_{4}\right)-4 a 1$ (k3\&.k4) (x2 \&.y2) (k3\&.y1)

$-4 a 1$ (k3\&.k4) (x2 \&.y2) (k4\&.y1) - 2 a2 ( x2 \&.y1) (k3\&.k4) (k2 \&.y2)

- 2 a2 ( k3 \&.y2) (x2 \&.y1) ( k3 \&.k4) - 2 a2 ( k4 \&.y2) ( x2 \&.y1) ( k3 \&.k4)

-2 a2 (y1 \&.y2) ( k3 \&.k4) ( k2 \&.x2) - 2 a2 ( k3 \&.x2) (y1 \&.y2) ( k3 \&.k4)

-2 a2 (k4\&.x2) (y1 \&.y2) ( k3 \&. k4 ) - 4 a1 (k3\&.k4) ( x2 \&.y2) ( k2 \&.y1)

-2 a3 (k3\&.y1) (x2 \&.y2) (k2\&.k4)

> unapply (expand $("(\mathrm{k} 3, \mathrm{k} 4) / 2+"(\mathrm{k} 4, \mathrm{k} 3) / 2), \mathrm{x} 2, \mathrm{y} 2)$;

$\left(x 2, y^{2}\right) \rightarrow$

-4a1 (k3\&.k4) (x2 \&.y2) ( k2 \&.y1) - 2 a3 (k4\&.y1) (x2 \&.y2) ( k2 \&. k3)

- 2 a3 ( k4 \&.y1) (x2 \&.y2) ( k3 \&.k3) - 2 a3 ( k4 \&.y1) ( x2 \&.y2) ( k3 \&.k4)

-2 a3 (k2 \&.y1) (k3 \&.x2) (k4\&.y2) - 2 a3 (k3 \&.x2) (k4\&.y2) (k3 \&.y1)

-2 a3 (k3\&.x2) (k4\&.y2) (k4\&.y1) - 2 a3 (k2 \&.y1) (k3\&.y2) (k4\&.x2)

-2 a3 (k3 \&.y2) (k4 \&.x2) (k3\&.y1) - 2 a3 (k3\&.y2) (k4 \&.x2) (k4 \&.y1)

$-a_{4}\left(y 1 \& . y_{2}\right)\left(k_{4} \& . x 2\right)\left(k_{2} \& . k_{3}\right)-a_{4}\left(y 1 \& . y_{2}\right)\left(k_{4} \& . x 2\right)\left(k 3 \& . k_{3}\right)$

$-a_{4}\left(k_{4} \& . x_{2}\right)\left(y 1 \& . y_{2}\right)\left(k_{3} \& . k_{4}\right)-a_{4}\left(k_{3} \& . y 1\right)\left(k_{4} \& . x_{2}\right)\left(k_{2} \& . y_{2}\right)$

$-a_{4}(k 3 \& . y 2)\left(k_{4} \& . x 2\right)(k 3 \& . y 1)-2 a_{4}(k 3 \& . y 1)\left(k_{4} \& . y 2\right)\left(k_{4} \& . x_{2}\right)$

$-a_{4}(k 3 \& . y 1)\left(k_{4} \& . y 2\right)(k 2 \& . x 2)-a_{4}(k 3 \& . x 2)(k 4 \& . y 2)(k 3 \& . y 1)$

$-a_{4}\left(x_{2} \& . y 1\right)\left(k_{4} \& . y 2\right)\left(k_{2} \& . k_{3}\right)-a_{4}\left(x_{2} \& . y 1\right)\left(k_{4} \& . y 2\right)\left(k 3 \& . k_{3}\right)$

$-a_{4}\left(k_{4} \& . y 2\right)\left(x_{2} \& . y 1\right)\left(k 3 \& . k_{4}\right)-a_{4}\left(x_{2} \& . y 1\right)(k 3 \& . y 2)\left(k 2 \& . k_{4}\right)$

$-a_{4}(k 3 \& . y 2)(x 2 \& . y 1)\left(k 3 \& . k_{4}\right)-a_{4}\left(x_{2} \& . y 1\right)(k 3 \& . y 2)\left(k_{4} \& . k_{4}\right)$

$-a_{4}\left(k_{4} \& . y 1\right)(k 3 \& . y 2)\left(k 2 \& . x_{2}\right)-2 a_{4}\left(k_{4} \& . y 1\right)(k 3 \& . y 2)(k 3 \& . x 2)$

- a4 (k3\&.y2) (k4\&.x2) (k4\&.y1)-a4 (k4\&.y1) (k3\&.x2) (k2 \&.y2)

$-a_{4}\left(k_{3} \& \cdot x_{2}\right)\left(k_{4} \& . y 2\right)\left(k_{4} \& \cdot y 1\right)-a_{4}\left(y 1 \& . y_{2}\right)\left(k_{3} \& . x_{2}\right)\left(k_{2} \& . k_{4}\right)$

$-a_{4}\left(k 3 \& . x_{2}\right)(y 1 \& . y 2)\left(k 3 \& . k_{4}\right)-a_{4}\left(y 1 \& . y_{2}\right)\left(k 3 \& . x_{2}\right)\left(k_{4} \& . k_{4}\right)$

$-4 a 1$ (k3\&.k4) (x2 \&.y2) (k3\&.y1) - 4 a1 (k3\&.k4) ( x2 \&.y2) (k4\&.y1) 
- 2 a2 ( x2 \&.y1) ( k3 \&.k4) ( k2 \&.y2) - 2 a2 ( k3 \&.y2) ( x2 \&.y1) ( k3 \&.k4)

-2 a2 (k4\&.y2) (x2 \&.y1) (k3\&.k4) - 2 a2 (y1 \&.y2) (k3\&.k4) ( k2 \&.x2)

-2 a2 ( k3 \&.x2) (y1 \&.y2) ( k3\&.k4) - 2 a2 ( k4 \&.x2) ( y1 \&.y2) (k3\&.k4)

-2 a3 (k3 \&.y1) (x2 \&.y2) ( k3 \&. k4 ) - 2 a3 ( k3 \&.y1) ( x2 \&.y2) (k4 \&. k4)

-2 a3 (k3\&.y1) (x2 \&.y2) (k2 \&. k4)

$>$ dS2c: $=$ expand $("(\mathrm{x} 2, \mathrm{y} 2) / 2+"(\mathrm{y} 2, \mathrm{x} 2) / 2)$;

dS2c $:=-4 a 1(k 3 \& . k 4)(x 2 \& . y 2)(k 2 \& . y 1)$

-2 a3 (k3\&.y1) (x2 \&.y2) (k2 \&. k4 ) - 2 a3 ( k3 \&.y1) (x2 \&.y2) (k3\&.k4)

-2 a3 ( k3 \&.y1) (x2 \&.y2) (k4\&.k4) - 2 a3 (k4\&.y1) ( x2 \&.y2) ( k2 \&. k3)

-2 a3 (k4 \&.y1) (x2 \&.y2) (k3\&.k3) - 2 a3 (k4\&.y1) (x2 \&.y2) (k3\&.k4)

-2 a3 (k2 \&.y1) (k3\&.x2) (k4\&.y2) - 2 a3 (k3\&.x2) (k4\&.y2) (k3\&.y1)

-2 a3 (k3\&.x2) (k4\&.y2) (k4\&.y1)-2a3 (k2\&.y1) (k3\&.y2) (k4\&.x2)

- 2 a3 (k3\&.y2) (k4\&.x2) (k3\&.y1) - 2 a3 (k3\&.y2) (k4 \&.x2) (k4\&.y1)

$-a_{4}\left(y 1 \& . y_{2}\right)\left(k_{4} \& . x 2\right)(k 2 \& . k 3)-a_{4}\left(y 1 \& . y_{2}\right)(k 4 \& . x 2)(k 3 \& . k 3)$

$-a_{4}\left(k_{4} \& . x_{2}\right)(y 1 \& . y 2)\left(k 3 \& . k_{4}\right)-a_{4}(k 3 \& . y 1)\left(k_{4} \& . x_{2}\right)\left(k 2 \& \cdot y_{2}\right)$

- a4 (k3\&.y2) (k4\&.x2) (k3\&.y1)-2a4 (k3\&.y1) (k4\&.y2) (k4\&.x2)

$-a_{4}(k 3 \& . y 1)(k 4 \& . y 2)(k 2 \& . x 2)-a 4(k 3 \& . x 2)(k 4 \& . y 2)(k 3 \& . y 1)$

$-a_{4}\left(x_{2} \& . y 1\right)\left(k_{4} \& . y 2\right)\left(k_{2} \& . k_{3}\right)-a_{4}\left(x_{2} \& . y 1\right)\left(k_{4} \& \cdot y_{2}\right)\left(k_{3} \& . k_{3}\right)$

$-a_{4}\left(k_{4} \& . y 2\right)\left(x_{2} \& \cdot y 1\right)\left(k 3 \& . k_{4}\right)-a_{4}\left(x_{2} \& . y 1\right)(k 3 \& . y 2)\left(k 2 \& . k_{4}\right)$

$-a_{4}(k 3 \& . y 2)(x 2 \& . y 1)\left(k 3 \& . k_{4}\right)-a_{4}\left(x_{2} \& . y 1\right)(k 3 \& . y 2)\left(k_{4} \& . k_{4}\right)$

$-a_{4}\left(k_{4} \& \cdot y 1\right)\left(k_{3} \& \cdot y_{2}\right)\left(k_{2} \& . x_{2}\right)-2 a_{4}\left(k_{4} \& . y 1\right)(k 3 \& . y 2)\left(k 3 \& . x_{2}\right)$

$-a_{4}\left(k_{3} \& . y_{2}\right)\left(k_{4} \& . x_{2}\right)\left(k_{4} \& . y 1\right)-a_{4}\left(k_{4} \& . y 1\right)\left(k_{3} \& . x_{2}\right)\left(k_{2} \& \cdot y_{2}\right)$

$-a_{4}\left(k_{3} \& . x_{2}\right)\left(k_{4} \& . y 2\right)\left(k_{4} \& . y 1\right)-a_{4}\left(y 1 \& . y_{2}\right)\left(k_{3} \& . x_{2}\right)\left(k_{2} \& . k_{4}\right)$

$-a_{4}\left(k 3 \& . x_{2}\right)(y 1 \& . y 2)\left(k 3 \& . k_{4}\right)-a_{4}\left(y 1 \& . y_{2}\right)\left(k 3 \& . x_{2}\right)\left(k_{4} \& . k_{4}\right)$

$-4 a 1$ (k3\&.k4) (x2 \&.y2) (k3\&.y1)-4a1 (k3\&.k4) (x2 \&.y2) (k4\&.y1)

-2 a2 (x2 \&.y1) (k3\&.k4) (k2 \&.y2) - 2 a2 ( k3 \&.y2) (x2 \&.y1) (k3\&.k4)

-2 a2 (k4 \&.y2) (x2 \&.y1) (k3\&.k4) - 2 a2 ( y1 \&.y2) (k3\&.k4) (k2 \&.x2)

-2 a2 (k3\&.x2) (y1 \&.y2) (k3\&.k4) - 2 a2 (k4 \&.x2) (y1 \&.y2) (k3\&.k4)

Vamos agora descobrir os valores das constantes que tornam essa variação nula. Quero dizer com isso, que teremos uma invariância na ação quando somarmos os termos de ordem um em $h_{\mu \nu}$, ou seja, a variação de ordem um dada por $S d 1 c+S d 2 c$, em que, teremos fatorizando a estrutura $k_{3}^{2} k_{4 \mu_{2}} \eta_{\nu_{1} \nu_{2}}$

$>$ factor $(\operatorname{coeff}(\operatorname{coeff}(\operatorname{expand}(\mathrm{dS} 1 \mathrm{c}+\mathrm{dS} 2 \mathrm{c}), \mathrm{y} 1 \& \cdot \mathrm{y} 2), \mathrm{k} 3 \& \cdot \mathrm{k} 3))$;

$$
-\frac{1}{4}\left(k_{4} \& \cdot x_{2}\right)\left(1+4 a_{4}\right)
$$


A condição necessária para o anulamento da variação é $a_{4}=-\frac{1}{4}$. Substituindo este valor na expressão abaixo, temos,

$>$ dnew: $=\operatorname{subs}(a 4=-1 / 4, d S 1 c+d S 2 c)$;

dnew $:=\frac{1}{4}\left(k_{4} \& \cdot y 1\right)\left(x_{2} \& \cdot y_{2}\right)(k 2 \& . k 3)$

$-4 a 1\left(k 3 \& . k_{4}\right)(x 2 \& . y 2)(k 2 \& . y 1)-\frac{1}{4}(k 2 \& . y 1)\left(k 3 \& . k_{4}\right)\left(x_{2} \& \cdot y 2\right)$

$+\frac{1}{4}(k 2 \& \cdot y 1)\left(k 3 \& \cdot x_{2}\right)\left(k_{4} \& \cdot y_{2}\right)+\frac{1}{4}(k 2 \& \cdot y 1)(k 3 \& \cdot y 2)\left(k 4 \& \cdot x_{2}\right)$

$+\frac{1}{4}\left(k_{3} \& \cdot y 1\right)\left(x_{2} \& \cdot y 2\right)\left(k_{2} \& \cdot k_{4}\right)+\frac{1}{4}\left(k_{3} \& \cdot y 1\right)\left(x_{2} \& \cdot y 2\right)\left(k_{4} \& \cdot k_{4}\right)$

$+\frac{1}{4}(y 1 \& . y 2)\left(k 3 \& . k_{4}\right)\left(\right.$ k2 \&.x2) $+\frac{1}{4}\left(k_{4} \& . y 1\right)(x 2 \& . y 2)(k 3 \& . k 3)$

$+\frac{1}{4}\left(x_{2} \& \cdot y 1\right)\left(k 3 \& . k_{4}\right)\left(k_{2} \& \cdot y_{2}\right)+\frac{1}{4}\left(k_{3} \& \cdot x_{2}\right)\left(k_{4} \& \cdot y_{2}\right)\left(k_{3} \& \cdot y 1\right)$

$+\frac{1}{4}\left(k_{3} \& \cdot x_{2}\right)\left(k_{4} \& \cdot y_{2}\right)\left(k_{4} \& \cdot y 1\right)+\frac{1}{4}\left(k_{3} \& \cdot y_{2}\right)\left(k_{4} \& \cdot x_{2}\right)\left(k_{3} \& \cdot y 1\right)$

$+\frac{1}{4}\left(k_{3} \& \cdot y_{2}\right)\left(k_{4} \& \cdot x_{2}\right)\left(k_{4} \& \cdot y 1\right)+\frac{1}{4}\left(k_{4} \& \cdot x_{2}\right)\left(y 1 \& \cdot y_{2}\right)\left(k_{3} \& \cdot k_{4}\right)$

$+\frac{1}{4}\left(k_{4} \& \cdot y 2\right)\left(x_{2} \& \cdot y 1\right)\left(k 3 \& \cdot k_{4}\right)+\frac{1}{4}(k 3 \& \cdot y 2)(x 2 \& \cdot y 1)\left(k 3 \& . k_{4}\right)$

$+\frac{1}{4}\left(k 3 \& . x_{2}\right)(y 1 \& . y 2)\left(k 3 \& . k_{4}\right)-2$ a3 (k3\&.y1) (x2 \&.y2) (k2 \&. k4)

-2 a3 ( k3 \&.y1) (x2 \&.y2) ( k3 \&. k4 ) - 2 a3 ( k3 \&.y1) ( x2 \&.y2) (k4\&.k4)

-2 a3 (k4\&.y1) (x2 \&.y2) (k2 \&. k3) - 2 a3 ( k4 \&.y1) ( x2 \&.y2) ( k3 \&.k3)

-2 a3 (k4\&.y1) (x2 \&.y2) ( k3 \&. k4 ) - 2 a3 ( k2 \&.y1) (k3\&.x2) (k4 \&.y2)

-2 a3 (k3 \&.x2) (k4 \&.y2) (k3\&.y1) - 2 a3 (k3 \&.x2) (k4\&.y2) (k4\&.y1)

-2 a3 (k2 \&.y1) (k3\&.y2) (k4\&.x2) - 2 a3 (k3\&.y2) (k4\&.x2) (k3\&.y1)

-2 a3 (k3\&.y2) (k4\&.x2) (k4\&.y1) - 4 a1 (k3\&.k4) (x2 \&.y2) (k3\&.y1)

$-4 a 1$ ( k3\&.k4) (x2 \&.y2) (k4\&.y1)-2 a2 (x2 \&.y1) (k3\&.k4) (k2 \&.y2)

-2 a2 (k3 \&.y2) (x2 \&.y1) (k3\&. k4) - 2 a2 ( k4 \&.y2) (x2 \&.y1) (k3\&.k4)

-2 a2 (y1 \&.y2) ( k3 \&.k4) (k2 \&.x2) - 2 a2 ( k3 \&.x2) (y1 \&.y2) ( k3 \&.k4)

-2 a2 (k4\&.x2) (y1\&.y2) (k3\&.k4).

Nos resta então calcular os valores das constantes $a_{3}, a_{1}$ e $a_{2}$, que são determinadas de forma análoga a realizada para encontrar $a_{4}$, logo temos,

$>$ factor $(\operatorname{coeff}(\operatorname{coeff}(\mathrm{dnew}, \mathrm{x} 2 \& \cdot \mathrm{y} 2), \mathrm{k} 3 \& . \mathrm{k} 3))$;

$$
-\frac{1}{4}(k 4 \& \cdot y 1)(-1+8 \text { a3 })
$$


$>$ dnew: $=\operatorname{expand}(\operatorname{subs}(a 3=1 / 8$, dnew $))$;

$$
\begin{aligned}
& \text { dnew }:=-\frac{1}{4}\left(k_{4} \& \cdot y 1\right)\left(x_{2} \& \cdot y 2\right)\left(k 3 \& \cdot k_{4}\right) \\
& -\frac{1}{4}(k 3 \& \cdot y 1)(x 2 \& \cdot y 2)\left(k 3 \& . k_{4}\right)-4 a 1\left(k 3 \& . k_{4}\right)(x 2 \& \cdot y 2)(k 2 \& \cdot y 1) \\
& -\frac{1}{4}(k 2 \& \cdot y 1)\left(k 3 \& . k_{4}\right)\left(x_{2} \& \cdot y_{2}\right)+\frac{1}{4}\left(y 1 \& \cdot y_{2}\right)\left(k 3 \& . k_{4}\right)\left(k 2 \& \cdot x_{2}\right) \\
& +\frac{1}{4}\left(x_{2} \& \cdot y 1\right)\left(k_{3} \& . k_{4}\right)\left(k_{2} \& \cdot y_{2}\right)+\frac{1}{4}\left(k_{4} \& . x_{2}\right)\left(y 1 \& \cdot y_{2}\right)\left(k_{3} \& . k_{4}\right) \\
& +\frac{1}{4}\left(k_{4} \& \cdot y_{2}\right)\left(x_{2} \& \cdot y 1\right)\left(k 3 \& . k_{4}\right)+\frac{1}{4}\left(k_{3} \& \cdot y 2\right)\left(x_{2} \& \cdot y 1\right)\left(k_{3} \& . k_{4}\right) \\
& +\frac{1}{4}\left(k_{3} \& . x_{2}\right)\left(y 1 \& . y_{2}\right)\left(k 3 \& . k_{4}\right)-4 a 1\left(k 3 \& . k_{4}\right)\left(x_{2} \& . y 2\right)(k 3 \& . y 1) \\
& \text { - } 4 a 1 \text { ( k3 \&.k4) (x2 \&.y2) (k4\&.y1) - } 2 \text { a2 ( x2 \&.y1) (k3\&.k4) (k2 \&.y2) } \\
& -2 \text { a2 ( k3 \&.y2) (x2 \&.y1) ( k3 \&. k4) - } 2 \text { a2 ( k4 \&.y2) ( x2 \&.y1) ( k3 \&.k4) } \\
& -2 \text { a2 (y1 \&.y2) ( k3 \&.k4) ( k2 \&.x2) - } 2 \text { a2 ( k3 \&.x2) (y1 \&.y2) ( k3 \&. k4 ) } \\
& -2 \text { a2 (k4\&.x2) (y1\&.y2) (k3\&.k4) }
\end{aligned}
$$

$>$ factor $(\operatorname{coeff}($ dnew, $x 2 \& \cdot y 2))$;

$$
-\frac{1}{4}(k 3 \& . k 4)(16 a 1+1)\left(\left(k_{4} \& . y 1\right)+(k 3 \& . y 1)+(k 2 \& . y 1)\right)
$$

$>$ dnew:=expand $(\operatorname{subs}(a 1=-1 / 16$, dnew $))$;

$$
\begin{aligned}
& \text { dnew }:=\frac{1}{4}(y 1 \& . y 2)(k 3 \& . k 4)(k 2 \& . x 2) \\
& +\frac{1}{4}\left(x_{2} \& \cdot y 1\right)\left(k_{3} \& . k_{4}\right)\left(k_{2} \& \cdot y_{2}\right)+\frac{1}{4}\left(k_{4} \& \cdot x_{2}\right)(y 1 \& . y 2)\left(k_{3} \& . k_{4}\right) \\
& +\frac{1}{4}\left(k_{4} \& \cdot y_{2}\right)\left(x_{2} \& \cdot y 1\right)\left(k 3 \& . k_{4}\right)+\frac{1}{4}\left(k_{3} \& . y 2\right)\left(x_{2} \& . y 1\right)\left(k 3 \& . k_{4}\right) \\
& +\frac{1}{4}\left(k_{3} \& . x_{2}\right)(y 1 \& . y 2)\left(k 3 \& . k_{4}\right)-2 \text { a2 (x2 \&.y1) (k3\&.k4) (k2 \&.y2) } \\
& \text { - } 2 \text { a2 ( k3 \&.y2) (x2 \&.y1) ( k3 \&. k4 ) - } 2 \text { a2 ( k4 \&.y2) ( x2 \&.y1) ( k3 \&. k4) } \\
& -2 \text { a2 (y1 \&.y2) ( k3 \&.k4) ( k2 \&.x2) - } 2 \text { a2 ( k3 \&.x2) ( y1 \&.y2) ( k3 \&.k4) } \\
& -2 \text { a2 (k4\&.x2) (y1\&.y2) (k3\&.k4) } \\
& >\operatorname{factor}(\operatorname{subs}(a 2=1 / 8, \text { dnew })) \text {; }
\end{aligned}
$$


APÊNDICE B. CÁLCULO DAS CONSTATES DO TENSOR $C^{(N)}$

Portanto, no caso comutativo, $\mathrm{a} 1=-1 / 16, \mathrm{a} 2=\mathrm{a} 3=1 / 8, \mathrm{a} 4=-1 / 4$

Para confirmarmos temos

$>\operatorname{expand}(\operatorname{subs}(\{a 1=-1 / 16, a 2=1 / 8, a 3=1 / 8, a 4=-1 / 4\}, d S 1 c+d S 2 c))$; 


\section{Referências Bibliográficas}

[1] H. Weyl, "Gravitation and electricity," Sitzungsber. Preuss. Akad. Wiss. Berlin (Math. Phys.) 1918 (1918) 465.

[2] V. Fock, "On the invariant form of the wave equations and the equations of motion for a charged point mass," Z. Phys. 39 (1926) 226-232.

[3] H. Weyl, "Electron and gravitation," Z. Phys. 56 (1929) 330-352.

[4] F. London, "Quantum mechanical interpretation of the Weyl's theory. (in german)," Z. Phys. 42 (1927) 375-389.

[5] C.-N. Yang and R. L. Mills, "Conservation of isotopic spin and isotopic gauge invariance," Phys. Rev. 96 (1954) 191-195.

[6] H. Yukawa, "On the interaction of elementary particles," Proc. Phys. Math. Soc. Jap. 17 (1935) 48-57.

[7] C. M. G. Lattes, G. P. S. Occhialini, and C. F. Powell, "Observations on the tracks of slow mesons in photographic emulsions. 1," Nature 160 (1947) 453-456.

[8] J. W. Moffat, "Perturbative noncommutative quantum gravity," Phys. Lett. B493 (2000) 142-148, hep-th/0008089.

[9] M. Gomes, "Remarks on noncommutative field theories," Braz. J. Phys. 34 (2004) 1330-1334.

[10] M. R. Douglas and N. A. Nekrasov, "Noncommutative field theory," Rev. Mod. Phys. 73 (2002) 977-1029, hep-th/0106048. 
[11] H. S. Snyder, "Quantized space-time," Phys. Rev. 71 (1947) 38-41.

[12] H. S. Snyder, "The eletromagnetic field in quantized space-time," Phys. Rev. 72 (1947) 68-71.

[13] S. Doplicher, K. Fredenhagen, and J. E. Roberts, "The quantum structure of space-time at the planck scale and quantum fields," Commun. Math. Phys. 172 (1995) 187-220, hep-th/0303037.

[14] N. Seiberg and E. Witten, "String theory and noncommutative geometry," JHPE 09 (1999) 032, hep-th/9908142.

[15] R. J. Szabo, "Quantum field theory on noncommutative spaces," Phys. Rept. 378 (2003) 207-299, hep-th/0109162.

[16] H. J. Groenewold, "On the principles of elementary quantum mechanics," Physica 12 (1946) 405-460.

[17] J. E. Moyal, "Quantum mechanics as a statistical theory," Proc. Cambridge Phil. Soc. 45 (1949) 99-124.

[18] S. Weinberg, Gravitation and Cosmology: Principles and Applications of the General Theory of Relativity. John Wiley \& Sons, NY, 1972.

[19] M. J. G. Veltman, "Quantum theory of gravitation,". In Les Houches 1975, Proceedings, Methods in Field Theory, Amsterdam 1976, 265-327.

[20] P. Aschieri et al., "A gravity theory on noncommutative spaces," Class. Quant. Grav. 22 (2005) 3511-3532, hep-th/0504183.

[21] P. Aschieri, M. Dimitrijevic, F. Meyer, and J. Wess, "Noncommutative geometry and gravity," Class. Quant. Grav. 23 (2006) 1883-1912, hep-th/0510059.

[22] X. Calmet and A. Kobakhidze, "Noncommutative general relativity," Phys. Rev. D72 (2005) 045010, hep-th/0506157. 
[23] X. Calmet and A. Kobakhidze, "Second order noncommutative corrections to gravity," hep-th/0605275.

[24] P. Mukherjee and A. Saha, "Comment on the first order noncommutative correction to gravity," hep-th/0605287.

[25] E. Harikumar and V. O. Rivelles, "Noncommutative gravity," hep-th/0607115.

[26] F. T. Brandt, J. Frenkel, and J. C. Taylor, "Metric dependence of partition function at high temperature," Nucl. Phys. B374 (1992) 169-182.

[27] S.-S. Chern and J. Simons, "Characteristic forms and geometric invariants," Annals Math. 99 (1974) 48-69.

[28] S. Deser, R. Jackiw, and S. Templeton, "Topologically massive gauge theories," Ann. Phys. 140 (1982) 372-411.

[29] R. P. Feynman, "Quantum theory of gravitation," Acta Phys. Polon. 24 (1963) 697-722.

[30] B. S. Dewitt, "Quantum theory of gravity. ii. the manifestly covariant theory," Phys. Rev. 162 (1967) 1195-1239.

[31] L. D. Faddeev and V. N. Popov, "Feynman diagrams for the yang-mills field," Phys. Lett. B25 (1967) 29-30.

[32] C. Becchi, A. Rouet, and R. Stora, "Renormalization of the Abelian Higgs-Kibble model," Commun. Math. Phys. 42 (1975) 127-162.

[33] I. V. Tyutin, "Gauge invariance in field theory and statistical physics in operator formalism,". LEBEDEV-75-39.

[34] J. Zinn-Justin, "Renormalization of gauge theories,". Lectures given at Int. Summer Inst. for Theoretical Physics, Jul 29 - Aug 9, 1974, Bonn, West Germany.

[35] K. Matsubara, "Restrictions on gauge groups in noncommutative gauge theory," Phys. Lett. B482 (2000) 417-419, hep-th/0003294. 
[36] J. Madore, S. Schraml, P. Schupp, and J. Wess, "Gauge theory on noncommutative spaces," Eur. Phys. J. C16 (2000) 161-167, hep-th/0001203.

[37] L. Bonora and M. Salizzoni, "Renormalization of noncommutative U(N) gauge theories," Phys. Lett. B504 (2001) 80-88, hep-th/0011088.

[38] A. Hsieh and E. Yehudai, "Hip: Symbolic high-energy physics calculations," Comput. Phys. 6 (1992) 253-261. 